\title{
From the Drinfeld Realization to the Drinfeld-Jimbo Presentation of Affine Quantum Algebras: Injectivity
}

by

\author{
Ilaria DAMIANI
}

\begin{abstract}
The surjective homomorphism $\psi$ (see [Da1]) from the Drinfeld realization $\mathcal{U}_{q}^{\mathrm{Dr}}$ to the Drinfeld and Jimbo presentation $\mathcal{U}_{q}^{\mathrm{DJ}}$ of affine quantum algebras is proved to be injective. A consequence of the arguments used in the paper is the triangular decomposition of the Drinfeld realization of affine quantum algebras also in the twisted case. A presentation of affine Kac-Moody algebras in terms of "Drinfeld generators" is also provided.
\end{abstract}

2010 Mathematics Subject Classification: Primary 17B37.

Keywords: quantum groups.

\section{§. Introduction}

Let $X_{\tilde{n}}^{(k)}$ be a Dynkin diagram of affine type, $\mathcal{U}_{q}^{\mathrm{DJ}}=\mathcal{U}_{q}^{\mathrm{DJ}}\left(X_{\tilde{n}}^{(k)}\right)$ the quantum algebra introduced by Drinfeld and Jimbo (see [Dr2] and [Jm]), and $\mathcal{U}_{q}^{\mathrm{Dr}}=\mathcal{U}_{q}^{\mathrm{Dr}}\left(X_{\tilde{n}}^{(k)}\right)$ its Drinfeld realization (see [Dr1]).

This paper concludes the proof that $\mathcal{U}_{q}^{\mathrm{DJ}}$ and $\mathcal{U}_{q}^{\mathrm{Dr}}$ are isomorphic. More precisely, in [Da1] a homomorphism $\psi: \mathcal{U}_{q}^{\mathrm{Dr}} \rightarrow \mathcal{U}_{q}^{\mathrm{DJ}}$ was defined (following [Be] for the untwisted case), and proved to be surjective; previous attempts to give a complete proof that these two algebras are isomorphic are also discussed in [Da1]. Here we prove the injectivity of $\psi$.

As in [Be], the idea of the proof is to recover the injectivity of $\psi$ from that of its specialization at 1 , based on the following:

Proposition 0.1. Let $\mathcal{A}=\mathbb{C}[q]_{(q-1)}$ be the localization of $\mathbb{C}[q]$ at $(q-1), M$ a finitely generated $\mathcal{A}$-module, $N$ a free $\mathcal{A}$-module, $f: M \rightarrow N$ a homomorphism of

Communicated by H. Nakajima. Received January 30, 2014. Revised June 10, 2014.

I. Damiani: Department of Mathematics, University of Rome "Tor Vergata", 00133 Roma, Italy; e-mail: damiani@mat.uniroma2.it

(C) 2015 Research Institute for Mathematical Sciences, Kyoto University. All rights reserved. 
$\mathcal{A}$-modules, $f_{1}: M /(q-1) M \rightarrow N /(q-1) N$ the $\mathcal{A} /(q-1)=\mathbb{C}$-linear homomorphism induced by $f$. If $f_{1}$ is injective, so is $f$.

Proof. $\mathcal{A}$ is a local principal ideal domain; $f(M)$ is a finitely generated $\mathcal{A}$-submodule of $N$, hence a free $\mathcal{A}$-module, so that there exists $g: f(M) \rightarrow M$ such that $f \circ g=\operatorname{id}_{f(M)}$.

Of course $\operatorname{ker}(f)$ is a finitely generated $\mathcal{A}$-module, $M=\operatorname{ker}(f) \oplus \operatorname{Im}(g)$, $\operatorname{ker}(f) /(q-1) \operatorname{ker}(f) \hookrightarrow M /(q-1) M$ and $\operatorname{ker}(f) /(q-1) \operatorname{ker}(f) \subseteq \operatorname{ker}\left(f_{1}\right)=\{0\}$.

Thus $(q-1) \operatorname{ker}(f)=\operatorname{ker}(f)$, so that $\operatorname{ker}(f)=\{0\}$ (Nakayama lemma).

Note that the hypothesis that $M$ is finitely generated over $\mathcal{A}$ is necessary, as can be seen from the simple counterexample $f: \mathbb{C}(q) \rightarrow\{0\}$.

The problem faced in the present paper is reducing to a situation where this argument works.

Consider the (well defined) commutative diagram

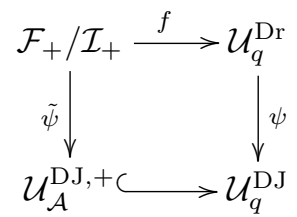

where $\mathcal{U}_{q}^{\mathrm{DJ}}$ and $\mathcal{U}_{q}^{\mathrm{Dr}}$ are respectively the Drinfeld-Jimbo presentation and the Drinfeld realization of a quantum affine algebra (see $\S 2$ and $\S 3$ ), $\mathcal{U}_{\mathcal{A}}^{\mathrm{DJ},+}$ is the integer form of the positive part of $\mathcal{U}_{q}^{\mathrm{DJ}}$ (Remark 2.4), $\mathcal{F}_{+}$is the free $\mathcal{A}$-algebra generated by $\left\{X_{i, r}^{+} \mid i \in I_{0}, r \geq 0\right\}$ and $\mathcal{I}_{+}$is the ideal of $\mathcal{F}_{+}$generated by the relations $\left(Z X_{+}^{+}, D R_{+}, S_{+}, U 3_{+}\right)$(see Notations 3.9 and 6.1).

The plan of the proof is to show that the injectivity of $\tilde{\psi}$ implies the injectivity of $\psi$ (see Proposition 5.2 and Corollary 6.4(ii)) and at the same time that the conditions of Proposition 0.1 hold for the homogeneous components of $\tilde{\psi}: \mathcal{F}_{+} / \mathcal{I}_{+} \rightarrow \mathcal{U}_{\mathcal{A}}^{\mathrm{DJ},+}$ (see Remark 6.7), so that $\psi$ is injective if $\tilde{\psi}_{1}$ (the specialization at 1 of $\tilde{\psi}$ ) is injective. This reduces our problem to the study of $\tilde{\psi}_{1}$, which is found to be injective through a careful analysis of the classical (non-quantum) affine Kac-Moody case (see Remark 7.6 and Corollary 8.21).

(A) It is well known that $\mathcal{U}_{\mathcal{A}}^{\mathrm{DJ},+}$ is a free $\mathcal{A}$-module (see Remark 2.4(ii)); it is straightforward to see that $\mathcal{F}_{+} / \mathcal{I}_{+}=\bigoplus_{\alpha \in Q_{+}}\left(\mathcal{F}_{+} / \mathcal{I}_{+}\right)_{\alpha}$ where each $\left(\mathcal{F}_{+} / \mathcal{I}_{+}\right)_{\alpha}$ is a finitely generated $\mathcal{A}$-module (see Remark 6.2(ii)). Finally $\tilde{\psi}$ is trivially $Q$ homogeneous; then Proposition 0.1 applies and $\tilde{\psi}$ is injective if $\tilde{\psi}_{1}$ is injective.

(B) Of course $\mathcal{F}_{+} / \mathcal{I}_{+}$, but also $\mathcal{U}_{\mathcal{A}}^{\mathrm{DJ},+}$, can be easily described through a presentation by generators and relations (it is well known that $\mathcal{U}_{\mathcal{A}}^{\mathrm{DJ},+}$ is generated by $\left\{E_{i} \mid i \in I\right\}$ with relations (SE), see Remark 2.4(iv)). Then their specializations 
at 1 are also immediate to describe by generators and relations (see Remarks 7.1 and 7.5$)$, and $\tilde{\psi}_{1}$ is explicitly known on the generators.

$\S 8$ is devoted to proving that $\tilde{\psi}_{1}$ is injective. Since the specialization at 1 of $\mathcal{U}_{\mathcal{A}}^{\mathrm{DJ},+}$ is well known (it is the enveloping algebra of the positive part of the KacMoody algebra), the proof consists in the study of the classical (non-quantum) situation, through a careful analysis of the specialization at 1 of $\mathcal{F}_{+} / \mathcal{I}_{+}$(see Corollary 7.29 and $\S 8$ ). In particular this analysis leads also to a "Drinfeld realization" of affine Kac-Moody algebras (see Theorem 9.6).

(C) On the other hand $f\left(\mathcal{F}_{+} / \mathcal{I}_{+}\right)$generates over $\mathbb{C}(q)$ a subalgebra $\mathcal{U}_{q}^{\mathrm{Dr},+,+}$ of $\mathcal{U}_{q}^{\mathrm{Dr},+} \subseteq \mathcal{U}_{q}^{\mathrm{Dr}}$; since $f\left(\mathcal{F}_{+} / \mathcal{I}_{+}\right)$is a direct sum of finitely generated $\mathcal{A}$-modules, it is an integer form of $\mathcal{U}_{q}^{\mathrm{Dr},+,+}$ (see Remark 6.3). So the injectivity of $\tilde{\psi}$ implies that $\left.\psi\right|_{\mathcal{U}_{q}^{\mathrm{Dr},+,+}}$ is injective (see Corollary 6.4(ii)).

But the injectivity of $\tilde{\psi}$ (hence of $f$ ) also implies that $\mathcal{F}_{+} / \mathcal{I}_{+} \cong f\left(\mathcal{F}_{+} / \mathcal{I}_{+}\right)$, that is, it provides a presentation by generators and relations of the integer form of $\mathcal{U}_{q}^{\text {Dr,+,++}}$ (see Corollary 6.4(i)).

(D) Why does the injectivity of $\left.\psi\right|_{\mathcal{U}_{q}^{\text {Dr, },+}}$ imply the injectivity of $\psi$ ? To answer this question we study the connection between the PBW-basis of $\mathcal{U}_{q}^{\mathrm{DJ}}$ and the tensor product $\mathcal{U}_{q}^{\mathrm{Dr},-,-} \otimes \mathcal{U}_{q}^{\mathrm{Dr}, 0} \otimes \mathcal{U}_{q}^{\mathrm{Dr},+,+}$ (see Proposition 5.1), recalling that $\mathcal{U}_{q}^{\text {Dr, }+}$ can be recovered from $\mathcal{U}_{q}^{\text {Dr, },++}$ by "translations" (see Remark 3.5(vii)).

With these tools it is easy to conclude finally that the injectivity of $\left.\psi\right|_{\mathcal{U}_{q}^{\mathrm{Dr},+,+}}$ implies the injectivity of $\psi$. At the same time it also implies the triangular decomposition of $\mathcal{U}_{q}^{\mathrm{Dr}}$ (see Proposition 5.2).

\section{$\S 1$. General notation}

We fix here the general notation that will be used in the paper (for a deeper and more detailed understanding of this setting see $[\mathrm{Bo}],[\mathrm{K}],[\mathrm{M}]$ ). Further notation is spread out through the next sections.

Notation 1.1. Following the literature we denote by

$$
\hat{\mathfrak{g}}=\left(\mathfrak{g} \otimes_{\mathbb{C}} \mathbb{C}\left[t^{ \pm 1}\right]\right)^{\chi} \oplus \mathbb{C} c \text { an affine Kac-Moody algebra }
$$

with

- Dynkin diagram $\Gamma$ and set of vertices $I=\{0,1, \ldots, n\}$,

- Cartan matrix $A=\left(a_{i j}\right)_{i, j \in I}$,

- root lattice $Q=\bigoplus_{i \in I} \mathbb{Z} \alpha_{i}$ and positive root lattice $Q_{+}=\bigoplus_{i \in I} \mathbb{N} \alpha_{i}$,

- root system (with real and imaginary roots) $\Phi=\Phi^{\mathrm{re}} \cup \Phi^{\mathrm{im}}$,

- root system with multiplicities $\hat{\Phi}$, 
- symmetric bilinear form $(\cdot \mid \cdot)$ on $Q$ induced by $D A\left(D=\operatorname{diag}\left(d_{i} \mid i \in I\right)\right)$ with kernel $\mathbb{Z} \delta\left(\delta \in Q_{+}\right)$,

- Weyl group $W=\left\langle s_{i}: \alpha_{j} \mapsto \alpha_{j}-a_{i j} \alpha_{i} \mid i \in I\right\rangle$,

- extended Weyl group $\hat{W}=W \rtimes \mathcal{T}(\mathcal{T} \leq \operatorname{Aut}(\Gamma))$ with length $l: \hat{W} \rightarrow \mathbb{N}$,

- extended braid group with lifting $\hat{W} \ni w \mapsto T_{w}$,

where

- $\mathfrak{g}$ is a simple Lie algebra over $\mathbb{C}$ of $\operatorname{rank} \tilde{n}$;

- $\chi$ is an automorphism of the Dynkin diagram of $\mathfrak{g}$ of order $k$;

and

- $A_{0}=\left(a_{i j}\right)_{i, j \in I_{0}}\left(I=I_{0} \cup\{0\} \neq I_{0}\right)$,

- $Q_{0}=\bigoplus_{i \in I_{0}} \mathbb{Z} \alpha_{i} \subseteq Q\left(Q_{0,+}=Q_{0} \cap Q_{+}\right)$,

- $\Phi_{0} \supseteq \Phi_{0,+}$ and

- $W_{0}=\left\langle s_{i} \mid i \in I_{0}\right\rangle \leq W \leq \hat{W}$

are respectively the Cartan matrix, the root lattice, the root system (with the set of positive roots) and the Weyl group of the simple Lie algebra $\mathfrak{g}_{0}=\mathfrak{g}^{\chi}$.

If $\mathfrak{g}$ is of type $X_{\tilde{n}}(X=A, B, C, D, E, F, G)$, then $\hat{\mathfrak{g}}$ is said to be of type $X_{\tilde{n}}^{(k)}$.

Finally $\hat{P}=\bigoplus_{i \in I_{0}} \mathbb{Z} \lambda_{i}\left(\left\langle\lambda_{i} \mid \alpha_{j}\right\rangle=\delta_{i j} \tilde{d}_{i}\right)$ is the sublattice of

$$
\operatorname{Hom}\left(Q_{0}, \mathbb{Z}\right) \subseteq \operatorname{Hom}\left(Q_{0}, \mathbb{Z}\right) \oplus \operatorname{Hom}(\mathbb{Z} \delta, \mathbb{Z})=\operatorname{Hom}\left(Q_{0} \oplus \mathbb{Z} \delta=Q, \mathbb{Z}\right)
$$

such that $\hat{W}=\hat{P} \rtimes W_{0}$ and

$$
\tilde{d}_{i}= \begin{cases}1 & \text { if } k=1 \text { or } X_{\tilde{n}}^{(k)}=A_{2 n}^{(2)}, \\ d_{i} & \text { otherwise; }\end{cases}
$$

recall that for all $\tilde{\lambda} \in \hat{P}$ and $\alpha \in Q, \tilde{\lambda}(\alpha)=\alpha-\langle\tilde{\lambda} \mid \alpha\rangle \delta$ and denote by $\lambda$ the weight $\lambda=\lambda_{1}+\cdots+\lambda_{n}$, by $N$ the length of $\lambda$, and by $N_{i}$ the length of $\lambda_{i}\left(i \in I_{0}\right)$.

Remark 1.2. The structure of the set $\hat{\Phi}$ of positive roots with multiplicities is the following (see $[K]): \hat{\Phi}=\Phi_{+}^{\mathrm{re}} \cup \hat{\Phi}_{+}^{\mathrm{im}}$ with

$$
\begin{aligned}
\Phi_{+}^{\mathrm{re}} & =\left\{r \delta+\alpha \in Q_{+} \mid \alpha \in \Phi_{0}, r \in \mathbb{Z} \text { such that } \tilde{d}_{\alpha} \mid r\right\} \cup \Phi_{2}, \\
\hat{\Phi}_{+}^{\mathrm{im}} & =\left\{(r \delta, i) \mid(i, r) \in I_{\mathbb{Z}}, r>0\right\},
\end{aligned}
$$

where

$$
\begin{aligned}
\tilde{d}_{w\left(\alpha_{i}\right)} & =\tilde{d}_{i} \quad \text { for } w \in W_{0}, i \in I_{0}, \\
\Phi_{2} & = \begin{cases}\left\{(2 r+1) \delta+2 \alpha \mid r \in \mathbb{N}, \alpha \in \Phi_{0},(\alpha \mid \alpha)=2\right\} & \text { in case } A_{2 n}^{(2)}, \\
\emptyset & \text { otherwise, }\end{cases} \\
I_{\mathbb{Z}} & =\left\{(i, r) \in I_{0} \times \mathbb{Z}\left|\tilde{d}_{i}\right| r\right\} .
\end{aligned}
$$


Notation 1.3 (see [Be] and [Da2]). The maps $\iota: \mathbb{Z} \rightarrow I$ and $\mathbb{Z} \ni r \mapsto w_{r} \in W$ are defined by the following conditions:

(i) $w_{r}= \begin{cases}s_{\iota_{1}} \cdot s_{\iota_{2}} \cdot \ldots \cdot s_{\iota_{r-1}} & \text { if } r \geq 1, \\ s_{\iota_{0}} \cdot s_{\iota_{-1}} \cdot \ldots \cdot s_{\iota_{r+1}} & \text { if } r \leq 0\end{cases}$

(ii) for all $r=1, \ldots, n$ there exists $\tau_{r} \in \mathcal{T}$ such that

$$
\lambda_{1}+\cdots+\lambda_{r}=\lambda_{1} \cdot \ldots \cdot \lambda_{r}=s_{\iota_{1}} \cdot \ldots \cdot s_{\iota_{N_{1}+\cdots+N_{r}}} \tau_{r} \in \hat{W}
$$

(iii) $\iota_{N+r}=\tau_{n}\left(\iota_{r}\right)$ for all $r \in \mathbb{Z}$.

The bijection $\mathbb{Z} \ni r \mapsto \beta_{r}=w_{r}\left(\alpha_{\iota_{r}}\right) \in \Phi_{+}^{\text {re }}$ induces a total ordering $\preceq$ on $\hat{\Phi}_{+}$ defined by

$$
\beta_{r} \preceq \beta_{r+1} \preceq(\tilde{m} \delta, i) \preceq(m \delta, j) \preceq(m \delta, i) \preceq \beta_{s-1} \preceq \beta_{s}
$$

for all $r \geq 1, s \leq 0, \tilde{m}>m>0, j \leq i \in I_{0}$ (choosing any ordering $\leq$ of $I_{0}$ ).

The reverse ordering has the same properties (see [Da2]).

Notation 1.4. (i) Consider the $\operatorname{ring} \mathbb{Z}\left[x, x^{-1}\right]$. Then for all $m, r \in \mathbb{Z}$ the elements $[m]_{x},[m]_{x} !(m \geq 0)$ and $\left[\begin{array}{c}m \\ r\end{array}\right]_{x}(m \geq r \geq 0)$ are defined by $[m]_{x}=$ $\frac{x^{m}-x^{-m}}{x-x^{-1}},[m]_{x} !=\prod_{s=1}^{m}[s]_{x}$ and $\left[\begin{array}{c}m \\ r\end{array}\right]_{x}=\frac{[m]_{x} !}{[r]_{x} ![m-r]_{x} !}$, which all lie in $\mathbb{Z}\left[x, x^{-1}\right]$.

(ii) Consider the field $\mathbb{C}(q)$ and, given $v \in \mathbb{C}(q) \backslash\{0\}$, the natural homomorphism $\mathbb{Z}\left[x, x^{-1}\right] \rightarrow \mathbb{C}(q)$ determined by the condition $x \mapsto v$; then for all $m, r \in \mathbb{Z}$ the elements $[m]_{v},[m]_{v} !(m \geq 0)$ and $\left[\begin{array}{c}m \\ r\end{array}\right]_{v},(m \geq r \geq 0)$ denote the images in $\mathbb{C}(q)$ of $[m]_{x},[m]_{x}$ ! and $\left[\begin{array}{c}m \\ r\end{array}\right]_{x}$ respectively.

(iii) For all $i \in I_{0}$ we denote $q_{i}=q^{d_{i}} \in \mathbb{C}(q)$.

Notation 1.5. Consider a $\mathbb{Z}\left[q^{ \pm 1}\right]$-algebra $U$, elements $u, v \in U$ and $r \in \mathbb{Z}$. The $q$-bracket $[u, v]_{q^{r}}$ is the element $[u, v]_{q^{r}}=u v-q^{r} v u$.

Note that the specialization at 1 of $[u, v]_{q^{r}}$ (the image of $[u, v]_{q^{r}}$ in the $\mathbb{Z}$-algebra $U /(q-1) U)$ is the classical bracket $[u, v]=u v-v u$.

\section{$\S 2$. Preliminaries: $\mathcal{U}_{q}^{\mathrm{DJ}}$}

In this section we recall the definition and the structures of the Drinfeld-Jimbo presentation $\mathcal{U}_{q}^{\mathrm{DJ}}$ of affine quantum algebras (see [Dr2] and [Jm], and also [Be], [Da2], [LS],[L]).

Definition 2.1. The Drinfeld-Jimbo presentation of the affine quantum algebra of type $X_{\tilde{n}}^{(k)}$ is the $\mathbb{C}(q)$-algebra $\mathcal{U}_{q}^{\mathrm{DJ}}=\mathcal{U}_{q}^{\mathrm{DJ}}\left(X_{\tilde{n}}^{(k)}\right)$ generated by

$$
\left\{E_{i}, F_{i}, K_{i}^{ \pm 1} \mid i \in I\right\}
$$


with relations

$$
\begin{gathered}
K_{i} K_{i}^{-1}=1=K_{i}^{-1} K_{i}, \quad K_{i} K_{j}=K_{j} K_{i} \quad \forall i, j \in I, \\
K_{i} E_{j}=q_{i}^{a_{i j}} E_{j} K_{i}, \quad K_{i} F_{j}=q_{i}^{-a_{i j}} F_{j} K_{i} \quad \forall i, j \in I, \\
{\left[E_{i}, F_{j}\right]=\delta_{i j} \frac{K_{i}-K_{i}^{-1}}{q_{i}-q_{i}^{-1}} \quad \forall i, j \in I,} \\
\sum_{u=0}^{1-a_{i j}}(-1)^{u}\left[\begin{array}{c}
1-a_{i j} \\
u
\end{array}\right]_{q_{i}} E_{i}^{u} E_{j} E_{i}^{1-a_{i j}-u}=0 \quad \forall i \neq j \in I, \\
\sum_{u=0}^{1-a_{i j}}(-1)^{u}\left[\begin{array}{c}
1-a_{i j} \\
u
\end{array}\right]_{q_{i}} F_{i}^{u} F_{j} F_{i}^{1-a_{i j}-u} \quad \forall i \neq j \in I .
\end{gathered}
$$

Remark 2.2. Recall that $\mathcal{U}_{q}^{\mathrm{DJ}}$ is endowed with the following structures:

(i) the $Q$-gradation $\mathcal{U}_{q}^{\mathrm{DJ}}=\bigoplus_{\alpha \in Q} \mathcal{U}_{q, \alpha}^{\mathrm{DJ}}$ determined by the conditions

$$
\begin{gathered}
E_{i} \in \mathcal{U}_{q, \alpha_{i}}^{\mathrm{DJ}}, \quad F_{i} \in \mathcal{U}_{q,-\alpha_{i}}^{\mathrm{DJ}}, \quad K_{i}^{ \pm 1} \in \mathcal{U}_{q, 0}^{\mathrm{DJ}} \quad \forall i \in I ; \\
\mathcal{U}_{q, \alpha}^{\mathrm{DJ}} \mathcal{U}_{q, \beta}^{\mathrm{DJ}} \subseteq \mathcal{U}_{q, \alpha+\beta}^{\mathrm{DJ}} \quad \forall \alpha, \beta \in Q ;
\end{gathered} \quad \forall
$$

(ii) the triangular decomposition

$$
\mathcal{U}_{q}^{\mathrm{DJ}} \cong \mathcal{U}_{q}^{\mathrm{DJ},-} \otimes \mathcal{U}_{q}^{\mathrm{DJ}, 0} \otimes \mathcal{U}_{q}^{\mathrm{DJ},+} \cong \mathcal{U}_{q}^{\mathrm{DJ},+} \otimes \mathcal{U}_{q}^{\mathrm{DJ}, 0} \otimes \mathcal{U}_{q}^{\mathrm{DJ},-},
$$

where $\mathcal{U}_{q}^{\mathrm{DJ},-}, \mathcal{U}_{q}^{\mathrm{DJ}, 0}$ and $\mathcal{U}_{q}^{\mathrm{DJ},+}$ are the subalgebras of $\mathcal{U}_{q}^{\mathrm{DJ}}$ generated respectively by $\left\{E_{i} \mid i \in I\right\},\left\{K_{i}^{ \pm 1} \mid i \in I\right\}$ and $\left\{F_{i} \mid i \in I\right\}$; in particular

$$
\mathcal{U}_{q, \alpha}^{\mathrm{DJ}} \cong \bigoplus_{\substack{\beta, \gamma \in Q_{+}: \\ \gamma-\beta=\alpha}} \mathcal{U}_{q,-\beta}^{\mathrm{DJ},-} \otimes \mathcal{U}_{q}^{\mathrm{DJ}, 0} \otimes \mathcal{U}_{q, \gamma}^{\mathrm{DJ},+} \quad \forall \alpha \in Q
$$

where $\mathcal{U}_{q, \alpha}^{\mathrm{DJ}, \pm}=\mathcal{U}_{q, \alpha}^{\mathrm{DJ}} \cap \mathcal{U}_{q}^{\mathrm{DJ}, \pm}$ is finite-dimensional for all $\alpha \in Q$; note also that if $\tilde{\mathcal{U}}_{q}^{\mathrm{DJ},-}=\bigoplus_{\alpha \in Q_{+}} \tilde{\mathcal{U}}_{q,-\alpha}^{\mathrm{DJ},-}$ with $\tilde{\mathcal{U}}_{q,-\alpha}^{\mathrm{DJ},-}=\mathcal{U}_{q,-\alpha}^{\mathrm{DJ},-} K_{\alpha}$, we have that $\tilde{\mathcal{U}}_{q}^{\mathrm{DJ},-}$ is a graded subalgebra of $\mathcal{U}_{q}^{\mathrm{DJ}}$ and the triangular decomposition can be formulated also as

$$
\mathcal{U}_{q}^{\mathrm{DJ}} \cong \tilde{\mathcal{U}}_{q}^{\mathrm{DJ},-} \otimes \mathcal{U}_{q}^{\mathrm{DJ}, 0} \otimes \mathcal{U}_{q}^{\mathrm{DJ},+} .
$$

(iii) the $\mathbb{C}$-anti-linear anti-involution $\Omega: \mathcal{U}_{q}^{\mathrm{DJ}} \rightarrow \mathcal{U}_{q}^{\mathrm{DJ}}$ defined by

$$
\Omega(q)=q^{-1}, \quad \Omega\left(E_{i}\right)=F_{i}, \quad \Omega\left(F_{i}\right)=E_{i}, \quad \Omega\left(K_{i}\right)=K_{i}^{-1} \quad \forall i \in I ;
$$

(iv) the extended braid group action defined by

$$
\begin{gathered}
T_{s_{i}}\left(K_{j}\right)=K_{j} K_{i}^{-a_{i j}} \quad \forall i, j \in I, \\
T_{s_{i}}\left(E_{i}\right)=-F_{i} K_{i}, \quad T_{s_{i}}\left(F_{i}\right)=-K_{i}^{-1} E_{i} \quad \forall i \in I,
\end{gathered}
$$


$T_{s_{i}}\left(E_{j}\right)=\sum_{r=0}^{-a_{i j}}(-1)^{r-a_{i j}} q_{i}^{-r} E_{i}^{\left(-a_{i j}-r\right)} E_{j} E_{i}^{(r)}, \quad T_{s_{i}}\left(F_{j}\right)=\Omega\left(T_{s_{i}}\left(E_{j}\right)\right)$

$\forall i \neq j \in I$

where $E_{i}^{(m)}=E_{i}^{m} /[m]_{q_{i}}$ ! for all $m \in \mathbb{N}$, and

$T_{\tau}\left(K_{i}\right)=K_{\tau(i)}, \quad T_{\tau}\left(E_{i}\right)=E_{\tau(i)}, \quad T_{\tau}\left(F_{i}\right)=F_{\tau(i)} \quad \forall \tau \in \operatorname{Aut}(\Gamma), i \in I_{0} ;$

(v) positive and negative root vectors $E_{\alpha} \in \mathcal{U}_{q, \alpha}^{\mathrm{DJ},+}$ and $F_{\alpha}=\Omega\left(E_{\alpha}\right) \in \mathcal{U}_{q,-\alpha}^{\mathrm{DJ},-}$ $\left(\alpha \in \hat{\Phi}_{+}\right)$such that $E_{\beta_{r}}=T_{w_{r}}\left(E_{\iota_{r}}\right)$ if $r \geq 1, E_{\beta_{r}}=T_{w_{r}^{-1}}^{-1}\left(E_{\iota_{r}}\right)$ if $r \leq 0$, and

$$
\exp \left(\left(q_{i}-q_{i}^{-1}\right) \sum_{r>0} E_{\left(\tilde{d}_{i} r \delta, i\right)} u^{r}\right)=1-\left(q_{i}-q_{i}^{-1}\right) \sum_{r>0} \tilde{E}_{\left(\tilde{d}_{i} r \delta, i\right)} u^{r}
$$

where $\tilde{E}_{\left(\tilde{d}_{i} r \delta, i\right)}=-E_{\tilde{d}_{i} r \delta-\alpha_{i}} E_{i}+q_{i}^{-2} E_{i} E_{\tilde{d}_{i} r \delta-\alpha_{i}}$ if $r>0$ and $i \in I_{0}$.

(Note that in [Da1], $E_{\left(\tilde{d}_{i} r \delta, i\right)}$ was confused with $\tilde{E}_{\left(\tilde{d}_{i} r \delta, i\right)}$ by a misprint.)

Remark 2.3. We have:

(i) $\Omega \circ T_{w}=T_{w} \circ \Omega$ for all $w \in \hat{W}$;

(ii) $T_{w}\left(\mathcal{U}_{q, \alpha}^{\mathrm{DJ}}\right)=\mathcal{U}_{q, w(\alpha)}^{\mathrm{DJ}}$ for all $w \in \hat{W}$ and $\alpha \in Q$;

(iii) $T_{w}\left(E_{i}\right), T_{w^{-1}}^{-1}\left(E_{i}\right) \in \mathcal{U}_{q}^{\mathrm{DJ},+}$ if $w \in \hat{W}$ and $i \in I$ are such that $w\left(\alpha_{i}\right) \in Q_{+}$;

(iv) $T_{w}\left(E_{i}\right) \in \tilde{\mathcal{U}}_{q}^{\mathrm{DJ},-}$ if $w \in \hat{W}$ and $i \in I$ are such that $w\left(\alpha_{i}\right) \in-Q_{+}$;

(v) $T_{w}\left(E_{i}\right)=E_{j}$ if $w \in \hat{W}$ and $i \in I$ are such that $w\left(\alpha_{i}\right)=\alpha_{j}$;

(vi) $T_{\lambda}\left(E_{\alpha}\right)= \begin{cases}E_{\lambda(\alpha)}=E_{\alpha-\langle\lambda \mid \alpha\rangle \delta} & \text { if } \lambda(\alpha) \in Q_{+}, \\ -F_{-\lambda(\alpha)} K_{-\lambda(\alpha)}=-F_{\langle\lambda \mid \alpha\rangle \delta-\alpha} K_{\langle\lambda \mid \alpha\rangle \delta-\alpha} & \text { otherwise; }\end{cases}$

(vii) $E_{r \tilde{d}_{i} \delta+\alpha_{i}}=T_{\lambda_{i}}^{-r}\left(E_{i}\right)$ for all $r \in \mathbb{N}$ and $i \in I_{0}$;

(viii) $T_{\lambda_{i}}\left(E_{\left(\tilde{d}_{j} r \delta, j\right)}\right)=E_{\left(\tilde{d}_{j} r \delta, j\right)}$ for all $i, j \in I_{0}$ and $r>0$;

(ix) $\left\{K_{\alpha}=\prod_{i \in I} K_{i}^{m_{i}} \mid \alpha=\sum_{i \in I} m_{i} \alpha_{i} \in Q\right\}$ is a basis of $\mathcal{U}_{q}^{\mathrm{DJ}, 0}$;

(x) $\left\{E(\gamma)=E_{\gamma_{1}} \cdot \ldots \cdot E_{\gamma_{M}} \mid M \in \mathbb{N}, \gamma=\left(\gamma_{1} \preceq \cdots \preceq \gamma_{M}\right), \gamma_{h} \in \hat{\Phi}_{+} \forall h=\right.$ $1, \ldots, M\}$ is a basis of $\mathcal{U}_{q}^{\mathrm{DJ},+}(\mathrm{PBW}$-basis $)$;

(xi) for all $\alpha \prec \beta \in \hat{\Phi}_{+}, E_{\beta} E_{\alpha}-q^{(\alpha \mid \beta)} E_{\alpha} E_{\beta}$ is a linear combination of $\{E(\gamma) \mid$ $\left.\gamma=\left(\gamma_{1} \preceq \cdots \preceq \gamma_{M}\right) \in \hat{\Phi}_{+}^{M}, M \in \mathbb{N}, \alpha \prec \gamma_{1}\right\}$; for real root vectors this can be stated in a more precise way: for all $\beta_{r} \prec \beta_{s} \in \hat{\Phi}_{+}$, $E_{\beta_{s}} E_{\beta_{r}}-q^{\left(\beta_{r} \mid \beta_{s}\right)} E_{\beta_{r}} E_{\beta_{s}}$ is a linear combination of $\left\{E(\gamma) \mid \gamma=\left(\gamma_{1} \preceq \ldots\right.\right.$ $\left.\left.\preceq \gamma_{M}\right) \in \hat{\Phi}_{+}^{M}, M \in \mathbb{N}, \beta_{r} \prec \gamma_{u} \prec \beta_{s} \forall u=1, \ldots, M\right\}$ (LevendorskiiSoibelman formula). 
Remark 2.4. The $\mathcal{A}$-subalgebra $\mathcal{U}_{\mathcal{A}}^{\mathrm{DJ}}$ of $\mathcal{U}_{q}^{\mathrm{DJ}}$ generated by $\left\{E_{i}, F_{i}, K_{i}^{ \pm 1} \mid i \in I\right\}$ is an integer form of $\mathcal{U}_{q}^{\mathrm{DJ}}$ :

(i) $\mathcal{U}_{q}^{\mathrm{DJ}}=\mathbb{C}(q) \otimes_{\mathcal{A}} \mathcal{U}_{\mathcal{A}}^{\mathrm{DJ}}$;

(ii) $\mathcal{U}_{\mathcal{A}}^{\mathrm{DJ}}$ is a free $\mathcal{A}$-module.

Moreover:

(iii) $\mathcal{U}_{\mathcal{A}}^{\mathrm{DJ}}$ is $T_{s_{i}}$-stable for all $i \in I$ and $T_{\tau}$-stable for all $\tau \in \mathcal{T}$; it contains all the root vectors;

(iv) $\mathcal{U}_{\mathcal{A}}^{\mathrm{DJ},+}=\mathcal{U}_{\mathcal{A}}^{\mathrm{DJ}} \cap \mathcal{U}_{q}^{\mathrm{DJ},+}$ is the $\mathcal{A}$-algebra generated by $\left\{E_{i} \mid i \in I\right\}$ with relations $(S E)$; it is a free $\mathcal{A}$-module;

(v) $\mathcal{U}_{\mathcal{A}}^{\mathrm{DJ},+}=\bigoplus_{\alpha \in Q_{+}} \mathcal{U}_{\mathcal{A}, \alpha}^{\mathrm{DJ},+}$ where $\mathcal{U}_{\mathcal{A}, \alpha}^{\mathrm{DJ},+}=\mathcal{U}_{\mathcal{A}}^{\mathrm{DJ},+} \cap \mathcal{U}_{q, \alpha}$ is free of finite rank over $\mathcal{A}$;

(vi) $\mathcal{U}_{\mathcal{A}}^{\mathrm{DJ}, 0}=\mathcal{U}_{\mathcal{A}}^{\mathrm{DJ}} \cap \mathcal{U}_{q}^{\mathrm{DJ}, 0}$ is the commutative $\mathcal{A}$-algebra

$$
\mathcal{U}_{\mathcal{A}}^{\mathrm{DJ}, 0}=\mathcal{A}\left[K_{i}, \frac{K_{i}-K_{i}^{-1}}{q_{i}-q_{i}^{-1}} \mid i \in I\right] /\left(K_{i}\left(K_{i}-\left(q_{i}-q_{i}^{-1}\right) \frac{K_{i}-K_{i}^{-1}}{q_{i}-q_{i}^{-1}}\right)-1 \mid i \in I\right) ;
$$

it is a free $\mathcal{A}$-module;

(vii) $\mathcal{U}_{\mathcal{A}}^{\mathrm{DJ}} /\left(q-1, K_{i}-1 \mid i \in I\right) \cong \mathcal{U}(\hat{\mathfrak{g}})$;

(viii) for all $i \in I, T_{s_{i}}$ induces $\tilde{T}_{s_{i}}: \mathcal{U}(\hat{\mathfrak{g}}) \rightarrow \mathcal{U}(\hat{\mathfrak{g}})$ and $\left.\tilde{T}_{s_{i}}\right|_{\hat{\mathfrak{g}}} \in \operatorname{Aut}_{\text {Lie }}(\hat{\mathfrak{g}})$; the image of all the root vectors lies in $\hat{\mathfrak{g}}$.

\section{§3. Preliminaries: $\mathcal{U}_{q}^{\mathrm{Dr}}$}

The Drinfeld realization $\mathcal{U}_{q}^{\mathrm{Dr}}$ of affine quantum algebras was introduced in [Dr1], and its defining relations were simplified in [Da1] thanks to the $(q-)$ commutation with the generators $X_{i, r}^{ \pm}, H_{i, r}$.

Both the original and the simplified sets of relations are useful in this paper: while studying the positive subalgebra $\mathcal{U}_{q}^{\mathrm{Dr},+}$, which contains neither $X_{i, r}^{-}$nor $H_{i, r}$, the set of relations given by Drinfeld is the most natural to deal with, and is finally proved to provide a complete set of relations defining $\mathcal{U}_{q}^{\mathrm{Dr},+}$ (see Theorem 9.4(i)); vice versa, specializing at 1 the whole $\mathcal{U}_{q}^{\text {Dr }}$ provides a presentation of affine KacMoody algebras in terms of the generators $\left\{x_{i, r}^{ \pm}, h_{i, r}, c\right\}$, whose relations can be deduced from the simplified relations defining $\mathcal{U}_{q}^{\text {Dr }}$ (see Theorem 9.6(iv)).

In this section we recall: the definition of $\mathcal{U}_{q}^{\text {Dr }}$ through the simplified relations given in [Da1] (Definition 3.1); the relations given by Drinfeld [Dr1] involving just the positive generators $X_{i, r}^{+}$'s and holding in $\mathcal{U}_{q}^{\text {Dr, }}$ (Notation 3.9 and Remark $3.10)$; the structures defined on $\mathcal{U}_{q}^{\mathrm{Dr}}$ ( $Q$-gradation, (anti)automorphisms, first remarks about triangular decomposition). 
Definition 3.1. The Drinfeld realization of the affine quantum algebra of type $X_{\tilde{n}}^{(k)}$ is the $\mathbb{C}(q)$-algebra $\mathcal{U}_{q}^{\mathrm{Dr}}=\mathcal{U}_{q}^{\mathrm{Dr}}\left(X_{\tilde{n}}^{(k)}\right)$ generated by

$$
C^{ \pm 1}, \quad k_{i}^{ \pm 1} \quad\left(i \in I_{0}\right), \quad X_{i, r}^{ \pm} \quad\left((i, r) \in I_{0} \times \mathbb{Z}\right)
$$

with relations

$$
X_{i, r}^{ \pm}=0 \quad \forall(i, r) \in\left(I_{0} \times \mathbb{Z}\right) \backslash I_{\mathbb{Z}},
$$

$$
[C, x]=0 \quad \forall x, \quad k_{i} k_{j}=k_{j} k_{i} \quad\left(i, j \in I_{0}\right),
$$

$$
C C^{-1}=1, \quad k_{i} k_{i}^{-1}=1=k_{i}^{-1} k_{i} \quad\left(i \in I_{0}\right)
$$

$$
k_{i} X_{j, r}^{ \pm}=q_{i}^{ \pm a_{i j}} X_{j, r}^{ \pm} k_{i} \quad\left(i \in I_{0},(j, r) \in I_{0} \times \mathbb{Z}\right),
$$

$$
\left[X_{i, r}^{+}, X_{j, s}^{-}\right]= \begin{cases}\delta_{i, j} \frac{C^{-s} k_{i} \tilde{H}_{i, r+s}^{+}-C^{-r} k_{i}^{-1} \tilde{H}_{i, r+s}^{-}}{q_{i}-q_{i}^{-1}} & \text { if } \tilde{d}_{j} \mid s \\ 0 & \text { otherwise }\end{cases}
$$

$\left(H X L^{ \pm}\right)$

$$
\begin{array}{ccc}
{\left[H_{i, r}, X_{j, s}^{ \pm}\right]= \pm b_{i j r} C^{(r \mp|r|) / 2} X_{j, r+s}^{ \pm}} & \left((i, r),(j, s) \in I_{\mathbb{Z}}, \tilde{d}_{i} \leq|r| \leq \tilde{d}_{i j}\right), \\
\left(X 1_{\text {const }}^{ \pm}\right) & {\left[X_{i, r \pm 1}^{ \pm}, X_{i, r}^{ \pm}\right]_{q^{2}}=0 \quad\left(X_{\tilde{n}}^{(k)}=A_{1}^{(1)}\right),} \\
\left(X 3_{\text {const }}^{ \pm}\right) & {\left[\left[X_{i, r \pm 1}^{ \pm}, X_{i, r}^{ \pm}\right]_{q^{2}}, X_{i, r}^{ \pm}\right]_{q^{4}}=0 \quad\left(X_{\tilde{n}}^{(k)}=A_{2}^{(2)}\right),} \\
\left(S_{\text {const }}^{ \pm}\right) & \sum_{u=0}^{1-a_{i j}}(-1)^{u}\left[\begin{array}{c}
1-a_{i j} \\
u
\end{array}\right]_{q_{i}}\left(X_{i, r}^{ \pm}\right)^{u} X_{j, s}^{ \pm}\left(X_{i, r}^{ \pm}\right)^{1-a_{i j}-u}=0 \quad(n>1),
\end{array}
$$

where $\tilde{H}_{i, r}^{ \pm}, H_{i, r}, b_{i j r}$ and $\tilde{d}_{i j}$ are defined as follows:

$$
\begin{aligned}
& \tilde{H}_{i, r}^{ \pm}= \begin{cases}1 & \text { if } r=0, \\
\pm\left(q_{i}-q_{i}^{-1}\right) C^{(r \mp r) / 2} k_{i}^{\mp 1}\left[X_{i, r}^{+}, X_{i, 0}^{-}\right] & \text {if } \pm r>0 \\
0 & \text { if } \pm r<0 ;\end{cases} \\
& \sum_{r \in \mathbb{Z}} \tilde{H}_{i, \pm r}^{ \pm} u^{r}=\exp \left( \pm\left(q_{i}-q_{i}^{-1}\right) \sum_{r>0} H_{i, \pm r} u^{r}\right) ; \\
& b_{i j r}= \begin{cases}0 & \text { if } \tilde{d}_{i, j} \nmid r, \\
{[2 r]_{q}\left(q^{2 r}+(-1)^{r-1}+q^{-2 r}\right) / r} & \text { if }\left(X_{\tilde{n}}^{(k)}, d_{i}, d_{j}\right)=\left(A_{2 n}^{(2)}, 1,1\right), \\
{\left[\tilde{r} a_{i j}\right]_{q_{i}} / \tilde{r}} & \text { otherwise, with } \tilde{r}=r / \tilde{d}_{i, j} ;\end{cases} \\
& \tilde{d}_{i j}=\max \left\{\tilde{d}_{i}, \tilde{d}_{j}\right\} .
\end{aligned}
$$

Notation 3.2. In $\mathcal{U}_{q}^{\mathrm{Dr}}$ :

(i) $\mathcal{U}_{q}^{\mathrm{Dr}, 0}$ denotes the $\mathbb{C}(q)$-subalgebra generated by $\left\{C^{ \pm 1}, k_{i}^{ \pm 1}, H_{i, r} \mid i \in I_{0}\right.$, $r \neq 0\}$, or equivalently, the $\mathbb{C}(q)$-subalgebra generated by $\left\{C^{ \pm 1}, k_{i}^{ \pm 1}, \tilde{H}_{i, r}^{ \pm}\right.$ $\left.i \in I_{0}, r \in \mathbb{Z}\right\}$ 
(ii) $\mathcal{U}_{q}^{\mathrm{Dr}, 0,0}, \mathcal{U}_{q}^{\mathrm{Dr}, 0,+}$ and $\mathcal{U}_{q}^{\mathrm{Dr}, 0,-}$ denote the $\mathbb{C}(q)$-subalgebras generated respectively by $\left\{C^{ \pm 1}, k_{i}^{ \pm 1} \mid i \in I_{0}\right\},\left\{H_{i, r} \mid i \in I_{0}, r>0\right\}$ (or $\left\{\tilde{H}_{i, r}^{+} \mid i \in I_{0}, r \in \mathbb{Z}\right\}$ ) and $\left\{H_{i, r} \mid i \in I_{0}, r<0\right\}$ (or $\left\{\tilde{H}_{i, r}^{-} \mid i \in I_{0}, r \in \mathbb{Z}\right\}$ );

(iii) $\mathcal{U}_{q}^{\mathrm{Dr},+}$ and $\mathcal{U}_{q}^{\mathrm{Dr},-}$ denote the $\mathbb{C}(q)$-subalgebras generated respectively by $\left\{X_{i, r}^{+} \mid i \in I_{0}, r \in \mathbb{Z}\right\}$ and $\left\{X_{i, r}^{-} \mid i \in I_{0}, r \in \mathbb{Z}\right\}$

(iv) $\mathcal{U}_{q}^{\mathrm{Dr},+,+}$ and $\mathcal{U}_{q}^{\mathrm{Dr},-,-}$ denote the $\mathbb{C}(q)$-subalgebras generated respectively by $\left\{X_{i, r}^{+} \mid i \in I_{0}, r \geq \mathbb{Z}\right\}$ and $\left\{X_{i, r}^{-} \mid i \in I_{0}, r \leq \mathbb{Z}\right\}$

(v) given $\alpha \in Q, \mathcal{U}_{q, \alpha}^{\mathrm{Dr}}$ denotes the $\alpha$-homogeneous component of $\mathcal{U}_{q}^{\mathrm{Dr}}: \mathcal{U}_{q}^{\mathrm{Dr}}=$ $\bigoplus_{\alpha \in Q} \mathcal{U}_{q, \alpha}^{\mathrm{Dr}}$ where $C^{ \pm 1}, k_{i}^{ \pm 1} \in \mathcal{U}_{q, 0}, X_{i, r}^{ \pm} \in \mathcal{U}_{q, r \delta \pm \alpha_{i}}^{\mathrm{Dr}} ; \mathcal{U}_{q, \alpha}^{\mathrm{Dr}, *}=\mathcal{U}_{q}^{\mathrm{Dr}, *} \cap \mathcal{U}_{q, \alpha}^{\mathrm{Dr}}$.

Remark 3.3. (i) $\mathcal{U}_{q}^{\mathrm{Dr}, 0,0} \subseteq \mathcal{U}_{q, 0}^{\mathrm{Dr}}$;

(ii) $\mathcal{U}_{q}^{\mathrm{Dr}, 0} \subseteq \bigoplus_{m \in \mathbb{Z}} \mathcal{U}_{q, m \delta}^{\mathrm{Dr}}$;

(iii) $\mathcal{U}_{q}^{\mathrm{Dr},+} \subseteq \mathbb{C}(q) \oplus \bigoplus_{m \in \mathbb{Z}, \alpha \in Q_{0,+} \backslash\{0\}} \mathcal{U}_{q, m \delta+\alpha}^{\mathrm{Dr}}$;

(iv) $\mathcal{U}_{q}^{\mathrm{Dr},+,+} \subseteq \mathbb{C}(q) \oplus \bigoplus_{m \in \mathbb{N}, \alpha \in Q_{0,+} \backslash\{0\}} \mathcal{U}_{q, m \delta+\alpha}^{\mathrm{Dr}}$;

(v) for all $\alpha \in Q_{0,+}$ and $m \in \mathbb{Z}, \mathcal{U}_{q, m \delta+\alpha}^{\mathrm{Dr},+,+}$ is finite-dimensional, while $\mathcal{U}_{q, m \delta+\alpha}^{\mathrm{Dr},+}$ is in general infinite-dimensional.

Definition 3.4. (i) $\Omega: \mathcal{U}_{q}^{\mathrm{Dr}} \rightarrow \mathcal{U}_{q}^{\mathrm{Dr}}$ is the $\mathbb{C}$-anti-linear anti-involution defined by

$$
\begin{gathered}
q \mapsto q^{-1}, \quad C^{ \pm 1} \mapsto C^{\mp 1}, \quad k_{i}^{ \pm 1} \mapsto k_{i}^{\mp 1}, \quad X_{i, r}^{ \pm} \mapsto X_{i,-r}^{\mp}, \\
\tilde{H}_{i, r}^{ \pm} \mapsto \tilde{H}_{i,-r}^{\mp}, \quad H_{i, r} \mapsto H_{i,-r} .
\end{gathered}
$$

(ii) $t_{i}: \mathcal{U}_{q}^{\mathrm{Dr}} \rightarrow \mathcal{U}_{q}^{\mathrm{Dr}}\left(i \in I_{0}\right)$ is the $\mathbb{C}(q)$-automorphism defined by

$$
\begin{gathered}
C^{ \pm 1} \mapsto C^{ \pm 1}, \quad k_{j}^{ \pm 1} \mapsto\left(k_{j} C^{-\delta_{i j} \tilde{d}_{i}}\right)^{ \pm 1}, \quad X_{j, r}^{ \pm} \mapsto X_{j, r \mp \delta_{i j} \tilde{d}_{i}}^{ \pm}, \\
\tilde{H}_{j, r}^{ \pm} \mapsto \tilde{H}_{j, r}^{ \pm}, \quad H_{j, r} \mapsto H_{j, r} .
\end{gathered}
$$

(iii) $\mathcal{E}_{c}: \mathcal{U}_{q}^{\mathrm{Dr}} \rightarrow \mathcal{U}_{q}^{\mathrm{Dr}}\left(c: I_{0} \rightarrow\{ \pm 1\}\right)$ is the $\mathbb{C}(q)$-automorphism defined by

$$
\left.\mathcal{E}_{c}\right|_{\mathcal{U}_{q}^{\mathrm{Dr}, 0}}=\mathrm{id}_{\mathcal{U}_{q}^{\mathrm{Dr}, 0}}, \quad X_{i, r}^{ \pm} \mapsto c_{i} X_{i, r}^{ \pm} .
$$

Remark 3.5. (i) For all $i, j \in I_{0}$ we have $\Omega \circ t_{i}=t_{i} \circ \Omega$ and $t_{i} \circ t_{j}=t_{j} \circ t_{i}$;

(ii) $\Omega\left(\mathcal{U}_{q}^{\mathrm{Dr}, 0}\right)=\mathcal{U}_{q}^{\mathrm{Dr}, 0}, \Omega\left(\mathcal{U}_{q}^{\mathrm{Dr}, 0,0}\right)=\mathcal{U}_{q}^{\mathrm{Dr}, 0,0}, \Omega\left(\mathcal{U}_{q}^{\mathrm{Dr}, 0, \pm}\right)=\mathcal{U}_{q}^{\mathrm{Dr}, 0, \mp}, \Omega\left(\mathcal{U}_{q}^{\mathrm{Dr}, \pm}\right)=$ $\mathcal{U}_{q}^{\mathrm{Dr}, \mp}, \Omega\left(\mathcal{U}_{q, \alpha}^{\mathrm{Dr}}\right)=\mathcal{U}_{q,-\alpha}^{\mathrm{Dr}}$

(iii) $t_{i}\left(\mathcal{U}_{q, \alpha}^{\mathrm{Dr}}\right)=\mathcal{U}_{q, \lambda_{i}(\alpha)}^{\mathrm{Dr}}$;

(iv) $t_{i}\left(\mathcal{U}_{q}^{\mathrm{Dr}, *}\right)=\mathcal{U}_{q}^{\mathrm{Dr}, *}, t_{i}\left(\mathcal{U}_{q}^{\mathrm{Dr}, 0, *}\right)=\mathcal{U}_{q}^{\mathrm{Dr}, 0, *}$

(v) more precisely, $\left.t_{i}\right|_{\mathcal{U}_{q}^{\mathrm{Dr}, 0, \pm}}=\mathrm{id}_{\mathcal{U}_{q}^{\mathrm{Dr}, 0, \pm}}(* \in\{0,+,-\})$;

(vi) $t_{i}^{-1}\left(\mathcal{U}_{q}^{\mathrm{Dr},+,+}\right) \subseteq \mathcal{U}_{q}^{\mathrm{Dr},+,+}$;

(vii) $\mathcal{U}_{q}^{\mathrm{Dr},+}=\bigcup_{N \in \mathbb{N}}\left(t_{1} \cdot \ldots \cdot t_{n}\right)^{N}\left(\mathcal{U}_{q}^{\mathrm{Dr},+,+}\right)$; 
(viii) for all $c, \tilde{c}: I_{0} \rightarrow\{ \pm 1\}$ and all $i \in I_{0}$ we have $\mathcal{E}_{c} \circ \mathcal{E}_{\tilde{c}}=\mathcal{E}_{c \tilde{c}}, \mathcal{E}_{c} \circ \Omega=\Omega \circ \mathcal{E}_{c}$ and $\mathcal{E}_{c} \circ t_{i}=t_{i} \circ \mathcal{E}_{c}$

(ix) $\mathcal{E}_{c}(x)= \pm x$ for all $x \in \mathcal{U}_{q, \alpha}^{\mathrm{Dr}}$ and $\alpha \in Q$.

Remark 3.6. In $\mathcal{U}_{q}^{\mathrm{Dr}}$ we also have (see [Dr1] and [Da1]):

$$
\begin{gathered}
\left(\begin{array}{c}
Z H) \\
H_{i, r}=0 \quad \forall(i, r) \in\left(I_{0} \times \mathbb{Z}\right) \backslash I_{\mathbb{Z}}, \\
(K H) \\
(H H)
\end{array} \quad\left[k_{i}, H_{j, s}\right]=0 \quad\left(i \in I_{0},(j, s) \in I_{0} \times(\mathbb{Z} \backslash\{0\})\right),\right. \\
\end{gathered}
$$

so that:

(i) $\mathcal{U}_{q}^{\mathrm{Dr}, 0,0}$ is central in $\mathcal{U}_{q}^{\mathrm{Dr}, 0}$;

(ii) $\mathcal{U}_{q}^{\mathrm{Dr}, 0,0}$ is a quotient of $\mathbb{C}(q)\left[C^{ \pm 1}, k_{i}^{ \pm 1} \mid i \in I_{0}\right]$ and $\mathcal{U}_{q}^{\mathrm{Dr}, 0,+}$ is a quotient of $\mathbb{C}(q)\left[H_{i, r}\left|i \in I_{0}, \tilde{d}_{i}\right| r>0\right]$;

(iii) the natural homomorphism of $\mathbb{C}(q)$-vector spaces

$$
\mathcal{U}_{q}^{\mathrm{Dr}, 0,-} \otimes_{\mathbb{C}(q)} \mathcal{U}_{q}^{\mathrm{Dr}, 0,0} \otimes_{\mathbb{C}(q)} \mathcal{U}_{q}^{\mathrm{Dr}, 0,+} \rightarrow \mathcal{U}_{q}^{\mathrm{Dr}, 0}
$$

is surjective.

Remark 3.7. In $\mathcal{U}_{q}^{\mathrm{Dr}}$ we also have (see [Dr1] and [Da1])

$\left(H X^{ \pm}\right) \quad\left[H_{i, r}, X_{j, s}^{ \pm}\right]= \pm b_{i j r} C^{(r \mp|r|) / 2} X_{j, r+s}^{ \pm} \quad\left((i, r),(j, s) \in I_{0} \times \mathbb{Z}, r \neq 0\right)$,

which, together with the relations $(C U K),(C K),\left(K X^{ \pm}\right),(X X)$, implies that the natural map $\mathcal{U}_{q}^{\mathrm{Dr},-} \otimes \mathcal{U}_{q}^{\mathrm{Dr}, 0} \otimes \mathcal{U}_{q}^{\mathrm{Dr},+} \rightarrow \mathcal{U}_{q}^{\mathrm{Dr}}$ is surjective.

Remark 3.8. Notice that setting $\tilde{\mathcal{U}}_{q}^{\mathrm{Dr},-}=\bigoplus_{\alpha \in Q} K_{\alpha} \mathcal{U}_{q, \alpha}^{\mathrm{Dr},-}\left(=\bigoplus_{\alpha \in Q} \mathcal{U}_{q, \alpha}^{\mathrm{Dr},-} K_{\alpha}\right)$ we get

$$
\tilde{\mathcal{U}}_{q}^{\mathrm{Dr},-} \otimes \mathcal{U}_{q}^{\mathrm{Dr}, 0} \otimes \mathcal{U}_{q}^{\mathrm{Dr},+} \cong \mathcal{U}_{q}^{\mathrm{Dr},-} \otimes \mathcal{U}_{q}^{\mathrm{Dr}, 0} \otimes \mathcal{U}_{q}^{\mathrm{Dr},+} .
$$

Notation 3.9. (i) Denote by $(D R)$ the following relations:

$$
\begin{aligned}
& {\left[X_{i, r+\tilde{d}_{i j}}^{+}, X_{j, s}^{+}\right]_{q_{i} a_{i j}}+\left[X_{j, s+\tilde{d}_{i j}}^{+}, X_{i, r}^{+}\right]_{q_{j}^{a_{j i}}}=0 \quad\left((i, r),(j, s) \in I_{\mathbb{Z}}, a_{i j}<0\right),} \\
& \sum_{\sigma \in \mathcal{S}_{2}} \sigma \cdot\left[X_{i, r_{1}+\tilde{d}_{i}}^{+}, X_{i, r_{2}}^{+}\right]_{q_{i}^{2}}=0 \quad\left(\left(r_{1}, r_{2}\right) \in \mathbb{Z}^{2},\left(X_{\tilde{n}}^{(k)}, d_{i}\right) \neq\left(A_{2 n}^{(2)}, 1\right)\right), \\
& (X 2) \quad \sum_{\sigma \in \mathcal{S}_{2}} \sigma \cdot\left(\left[X_{i, r_{1}+2}^{+}, X_{i, r_{2}}^{+}\right]_{q^{2}}-q^{4}\left[X_{i, r_{1}+1}^{+}, X_{i, r_{2}+1}^{+}\right]_{q^{-6}}\right)=0 \\
& \left(\left(r_{1}, r_{2}\right) \in \mathbb{Z}^{2},\left(X_{\tilde{n}}^{(k)}, d_{i}\right)=\left(A_{2 n}^{(2)}, 1\right)\right), \\
& \sum_{\sigma \in \mathcal{S}_{3}} \sigma \cdot\left[\left[X_{i, r_{1}+1}^{+}, X_{i, r_{2}}^{+}\right]_{q^{2}}, X_{i, r_{3}}^{+}\right]_{q^{4}}=0 \\
& \left(\left(r_{1}, r_{2}, r_{3}\right) \in \mathbb{Z}^{3},\left(X_{\tilde{n}}^{(k)}, d_{i}\right)=\left(A_{2 n}^{(2)}, 1\right)\right),
\end{aligned}
$$


$(S U L)$

$$
\begin{array}{r}
\sum_{\sigma \in \mathcal{S}_{1-a_{i j}}} \sigma \cdot\left[\left[\ldots\left[\left[X_{j, s}^{+}, X_{i, r_{1}}^{+}\right]_{q_{i}^{-a_{i j}}}, X_{i, r_{2}}^{+}\right]_{q_{i}^{-a_{i j}-2}}, \ldots\right]_{q_{i}^{a_{i j}+2}}, X_{i, r_{1-a_{i j}}}^{+}\right]_{q_{i} a_{i j}}=0 \\
\left(i \neq j \in I_{0}, a_{i j} \in\{0,-1\} \text { if } k \neq 1, r \in \mathbb{Z}^{1-a_{i j}}, s \in \mathbb{Z}\right),
\end{array}
$$

$$
\begin{aligned}
& \sum_{\sigma \in \mathcal{S}_{2}} \sigma \cdot\left[\left[X_{j, s}^{+}, X_{i, r_{1}+1}^{+}\right]_{q^{2}}, X_{i, r_{2}}^{+}\right]=0, \\
& \quad\left(i, j \in I_{0}, a_{i j}=-2, k=2, X_{\tilde{n}}^{(k)} \neq A_{2 n}^{(2)},\left(r_{1}, r_{2}\right) \in \mathbb{Z}^{2}, s \in \mathbb{Z}\right),
\end{aligned}
$$

$$
\begin{aligned}
\sum_{\sigma \in \mathcal{S}_{2}} \sigma \cdot\left(\left(q^{2}+q^{-2}\right)\left[\left[X_{j, s}^{+}, X_{i, r_{1}+1}^{+}\right]_{q^{2}}, X_{i, r_{2}}^{+}\right]\right. & \\
& \left.+q^{2}\left[\left[X_{i, r_{1}+1}^{+}, X_{i, r_{2}}^{+}\right]_{q^{2}}, X_{j, s}^{+}\right]_{q^{-4}}\right)=0 \\
\left(i, j \in I_{0}, a_{i j}\right. & \left.=-2, X_{\tilde{n}}^{(k)}=A_{2 n}^{(2)},\left(r_{1}, r_{2}\right) \in \mathbb{Z}^{2}, s \in \mathbb{Z}\right),
\end{aligned}
$$

$$
\begin{aligned}
& \sum_{\sigma \in \mathcal{S}_{2}} \sigma \cdot\left(( q ^ { 2 } + 1 ) \left[\left[X_{j, s}^{+}, X_{i, r_{1}+2}^{+}\right]_{q^{3}},\right.\right.\left.X_{i, r_{2}}^{+}\right]_{q^{-1}} \\
&\left.+\left[\left[X_{j, s}^{+}, X_{i, r_{1}+1}^{+}\right]_{q^{3}}, X_{i, r_{2}+1}^{+}\right]_{q}\right)=0 \\
&\left(i, j \in I_{0}, a_{i j}=-3, k=3,\left(r_{1}, r_{2}\right) \in \mathbb{Z}^{2}, s \in \mathbb{Z}\right) .
\end{aligned}
$$

(ii) Denote by $(S)$ the relations

$$
\begin{array}{r}
\sum_{\sigma \in \mathcal{S}_{1-a_{i j}}} \sigma \cdot\left[\ldots\left[\left[X_{j, s}^{+}, X_{i, r_{1}}^{+}\right]_{q_{i}}^{-a_{i j}}, X_{i, r_{2}}^{+}\right]_{q_{i}^{-}} a_{i j}-2, \ldots, X_{i, r_{1-a_{i j}}}^{+}\right]_{q_{i}} a_{i j}=0 \\
\left(i \neq j \in I_{0}, r \in \mathbb{Z}^{1-a_{i j}}, s \in \mathbb{Z}\right) .
\end{array}
$$

(iii) Denote by $(U 3)$ the relations

$$
\begin{aligned}
\sum_{\sigma \in \mathcal{S}_{3}} \sigma \cdot\left[\left[\left[X_{j, s}^{+}, X_{i, r_{1}+1}^{+}\right]_{q^{3}}, X_{i, r_{2}}^{+}\right]_{q}, X_{i, r_{3}}^{+}\right]_{q^{-1}} & =0 \\
\left(i, j \in I_{0}, a_{i j}=-3, k\right. & \left.=3,\left(r_{1}, r_{2}\right) \in \mathbb{Z}^{2}, s \in \mathbb{Z}\right) .
\end{aligned}
$$

(iv) Denote by $\left(Z X_{+}^{+}\right)$, respectively $\left(D R_{+}\right),\left(S_{+}\right)$and $\left(U 3_{+}\right)$, the relations of $\left(Z X^{+}\right)$, respectively $(D R),(S)$ and $(U 3)$, involving just elements $X_{i, r}^{+}$with $r \geq 0$ (see Definition 3.1).

Remark 3.10. (i) The relations $(D R),(S)$ and $(U 3)$ hold in $\mathcal{U}_{q}^{\mathrm{Dr}}$ (see [Dr1] and [Da1]);

(ii) the relations $(S U L)$ and $\left(S U L_{+}\right)$depend on $(S)$ and $\left(S_{+}\right)$respectively;

(iii) the relations $(S)$ and $(U 3)$ depend on $(D R)$ (see [Da1]);

(iv) in the algebra generated by $\left\{X_{i, r}^{+} \mid i \in I_{0}, r \in \mathbb{N}\right\}$ the relations $\left(S_{+}\right)$and $\left(U 3_{+}\right)$do not depend on $\left(D R_{+}\right)$(it is enough to compare the degrees of $\left(S_{+}\right)$ and $\left(U 3_{+}\right)$with those of $\left(D R_{+}\right)$remarking that the algebra generated by $\left\{X_{i, r}^{+} \mid i \in I_{0}, r \in \mathbb{N}\right\}$ is $Q_{0,+} \oplus \mathbb{N} \delta$-graded). 


\section{§4. Preliminaries: $\psi$}

In this section we recall the homomorphism $\psi: \mathcal{U}_{q}^{\mathrm{Dr}} \rightarrow \mathcal{U}_{q}^{\mathrm{DJ}}$ and some of its properties (see [Be] and [Da2]).

Definition 4.1. $\psi=\psi_{X_{\tilde{n}}^{(k)}}: \mathcal{U}_{q}^{\mathrm{Dr}}\left(X_{\tilde{n}}^{(k)}\right) \rightarrow \mathcal{U}_{q}^{\mathrm{DJ}}\left(X_{\tilde{n}}^{(k)}\right)$ is the $\mathbb{C}(q)$-algebra homomorphism defined on generators as follows:

$$
\begin{gathered}
C^{ \pm 1} \mapsto K_{\delta}^{ \pm 1}, \quad k_{i}^{ \pm 1} \mapsto K_{i}^{ \pm 1} \quad\left(i \in I_{0}\right), \\
X_{i, \tilde{d}_{i} r}^{+} \mapsto o(i)^{r} T_{\lambda_{i}}^{-r}\left(E_{i}\right), \quad X_{i, \tilde{d}_{i} r}^{-} \mapsto o(i)^{r} T_{\lambda_{i}}^{r}\left(F_{i}\right) \quad\left(i \in I_{0}, r \in \mathbb{Z}\right), \\
H_{i, \tilde{d}_{i} r} \mapsto\left\{\begin{array}{ll}
o(i)^{r} E_{\left(\tilde{d}_{i} r \delta, i\right)} & \text { if } r>0 \\
o(i)^{r} F_{\left(-\tilde{d}_{i} r \delta, i\right)} & \text { if } r<0
\end{array} \quad\left(i \in I_{0}, r \in \mathbb{Z} \backslash\{0\}\right),\right.
\end{gathered}
$$

where $o: I_{0} \rightarrow\{ \pm 1\}$ is a map such that:

(a) $a_{i j} \neq 0 \Rightarrow o(i) o(j)=-1$ (see [Be] for the untwisted case);

(b) in the twisted case different from $A_{2 n}^{(2)}, a_{i j}=-2 \Rightarrow o(i)=1$ (see [Da1]).

Remark 4.2 (see [Da1]). (i) $\psi$ preserves the gradation, that is, $\psi\left(\mathcal{U}_{q, \alpha}^{\mathrm{Dr}}\right)=\mathcal{U}_{q, \alpha}^{\mathrm{DJ}}$ for all $\alpha \in Q$;

(ii) $\psi \circ \Omega=\Omega \circ \psi$;

(iii) $\psi \circ \mathcal{E}_{O_{i}} \circ t_{i}=T_{\lambda_{i}} \circ \psi$ for all $i \in I_{0}$, where

$$
o_{i}(j)= \begin{cases}o(i) & \text { if } j=i \\ 1 & \text { otherwise }\end{cases}
$$

(iv) $\psi$ is surjective.

Proposition 4.3. Let us compare $\mathcal{U}_{q}^{\mathrm{Dr}}$ and $\mathcal{U}_{q}^{\mathrm{DJ}}$ through $\psi$; then the $P B W$-basis of $\mathcal{U}_{q}^{\text {DJ }}$ and Remark 3.6(ii)\&(iii) imply that:

(i) $\mathcal{U}_{q}^{\mathrm{Dr}, 0,0} \cong \mathbb{C}(q)\left[C^{ \pm 1}, k_{i}^{ \pm 1} \mid i \in I_{0}\right]$ and $\left.\psi\right|_{\mathcal{U}_{q}^{\mathrm{Dr}, 0,0}}: \mathcal{U}_{q}^{\mathrm{Dr}, 0,0} \rightarrow \mathcal{U}_{q}^{\mathrm{DJ}, 0}$ is an isomorphism;

(ii) $\mathcal{U}_{q}^{\mathrm{Dr}, 0,+} \cong \mathbb{C}(q)\left[H_{i, r}\left|i \in I_{0}, \tilde{d}_{i}\right| r>0\right]$ and $\mathcal{U}_{q}^{\mathrm{Dr}, 0,-} \cong \mathbb{C}(q)\left[H_{i, r} \mid i \in I_{0}\right.$, $\left.\tilde{d}_{i} \mid r<0\right]$

(iii) the composition

$$
\mathcal{U}_{q}^{\mathrm{Dr}, 0,-} \otimes_{\mathbb{C}(q)} \mathcal{U}_{q}^{\mathrm{Dr}, 0,0} \otimes_{\mathbb{C}(q)} \mathcal{U}_{q}^{\mathrm{Dr}, 0,+} \rightarrow \mathcal{U}_{q}^{\mathrm{Dr}, 0} \hookrightarrow \mathcal{U}_{q}^{\mathrm{Dr}} \stackrel{\psi}{\rightarrow} \mathcal{U}_{q}^{\mathrm{DJ}}
$$

is injective;

(iv) $\mathcal{U}_{q}^{\mathrm{Dr}, 0,-} \otimes_{\mathbb{C}(q)} \mathcal{U}_{q}^{\mathrm{Dr}, 0,0} \otimes_{\mathbb{C}(q)} \mathcal{U}_{q}^{\mathrm{Dr}, 0,+} \cong \mathcal{U}_{q}^{\mathrm{Dr}, 0}$;

(v) $\left.\psi\right|_{\mathcal{U}_{q}^{\mathrm{Dr}, 0}}: \mathcal{U}_{q}^{\mathrm{Dr}, 0} \rightarrow \mathcal{U}_{q}^{\mathrm{DJ}}$ is injective. 


\section{$\S 5$. Reduction to a finite-dimensional situation and triangular decomposition}

The aim of this paper is to prove that $\psi$ is an isomorphism, i.e. that it is injective (since it is surjective). The strategy is to reduce the task to studying the restriction of $\psi$ to finitely generated $\mathcal{A}$-submodules of $\mathcal{U}_{q}^{\mathrm{Dr}}$, so that the specialization argument described in the introduction (Proposition 0.1) can be applied.

The first step in this direction would be restricting to the $Q$-homogeneous components $\mathcal{U}_{q, \alpha}^{\mathrm{Dr}}$, which are though far from being finite-dimensional; in similar situations, for example when studying the Drinfeld-Jimbo presentation of quantum algebras, triangular decomposition solves this difficulty, because it provides the lower bound $0 \in Q$ for the weight of the elements considered.

In the Drinfeld realization this simplification is important but not enough: indeed, $\mathcal{U}_{q, \alpha}^{\mathrm{Dr},+}$ is in general infinite-dimensional (see Remark 3.3(v)). The same remark suggests analyzing in fact $\mathcal{U}_{q}^{\mathrm{Dr},+,+}$ since it is the direct sum of (its homogeneous) finite-dimensional components.

This section is devoted to showing that the injectivity of $\left.\psi\right|_{\mathcal{U}_{q}^{\mathrm{Dr},+,+}}$ implies the injectivity of $\psi$.

As outlined above, the reduction to this finite-dimensional situation requires the analysis and understanding of the triangular decomposition of $\mathcal{U}_{q}^{\mathrm{Dr}}$.

By triangular decomposition of $\mathcal{U}_{q}^{\text {Dr }}$ we mean the following claim:

$$
\mathcal{U}_{q}^{\mathrm{Dr},-} \otimes_{\mathbb{C}(q)} \mathcal{U}_{q}^{\mathrm{Dr}, 0} \otimes_{\mathbb{C}(q)} \mathcal{U}_{q}^{\mathrm{Dr},+} \cong \mathcal{U}_{q}^{\mathrm{Dr}} .
$$

Hernandez $[\mathrm{H}]$ proved triangular decomposition for the quantum affinizations of all symmetrizable quantum algebras; this class of algebras includes the untwisted affine quantum algebras, but does not include the twisted ones.

Here we show that the injectivity of $\left.\psi\right|_{\mathcal{U}_{q}^{\mathrm{Dr},+,+}}$ implies both the triangular decomposition of $\mathcal{U}_{q}^{\text {Dr }}$ and the injectivity of $\psi$.

We have already noticed that the product $\mathcal{U}_{q}^{\mathrm{Dr},-} \otimes_{\mathbb{C}(q)} \mathcal{U}_{q}^{\mathrm{Dr}, 0} \otimes_{\mathbb{C}(q)} \mathcal{U}_{q}^{\mathrm{Dr},+} \rightarrow \mathcal{U}_{q}^{\mathrm{Dr}}$ is surjective (see Remark 3.7): therefore triangular decomposition is equivalent to the injectivity of this map.

Proposition 5.1. The product map $\psi\left(\mathcal{U}_{q}^{\mathrm{Dr},-,-}\right) \otimes_{\mathbb{C}(q)} \psi\left(\mathcal{U}_{q}^{\mathrm{Dr}, 0}\right) \otimes_{\mathbb{C}(q)} \psi\left(\mathcal{U}_{q}^{\mathrm{Dr},+,+}\right)$ $\rightarrow \mathcal{U}_{q}^{\mathrm{DJ}}$ is injective.

Proof. $\psi\left(\mathcal{U}_{q}^{\mathrm{Dr},+,+}\right)$ is the subalgebra of $\mathcal{U}_{q}^{\mathrm{DJ}}$ generated by the root vectors $E_{r \delta+\alpha_{i}}$ $\left(i \in I_{0}, r \in \mathbb{N}\right.$ ), hence, by the Levendorskii-Soibelman formula and the PBW-basis (see Remark 2.3(x)\&(xi)), it is a subspace of the linear span of the ordered monomials in the root vectors $E_{\beta_{r}}$ with $r \leq 0$. Of course $\psi\left(\mathcal{U}_{q}^{\mathrm{Dr},-,-}\right)=\Omega\left(\psi\left(\mathcal{U}_{q}^{\mathrm{Dr},+,+}\right)\right)$, hence it is a subspace of the linear span of the ordered monomials in the root vectors $F_{\beta_{r}}$ with $r \leq 0$. 
Recall that $\psi\left(\mathcal{U}_{q}^{\mathrm{Dr}, 0}\right) \cong \psi\left(\mathcal{U}_{q}^{\mathrm{Dr}, 0,-}\right) \otimes \psi\left(\mathcal{U}_{q}^{\mathrm{Dr}, 0,0}\right) \otimes \psi\left(\mathcal{U}_{q}^{\mathrm{Dr}, 0,+}\right)$ (see Proposition $4.3(\mathrm{iv}) \&(\mathrm{v}))$, and that $\psi\left(\mathcal{U}_{q}^{\mathrm{Dr}, 0,+}\right)$ is the subalgebra of $\mathcal{U}_{q}^{\mathrm{DJ}}$ generated by the root vectors $E_{(r \delta, i)}\left(i \in I_{0}, r>0\right)$.

Then the triangular decomposition of $\mathcal{U}_{q}^{\mathrm{DJ}}$ (see Remark 2.2(ii)) and the structure of its PBW-basis (see Remark 2.3(x)) imply the assertion, thanks to Proposition $4.3(\mathrm{i}) \&(\mathrm{ii})$.

Proposition 5.2. If $\left.\psi\right|_{\mathcal{U}_{q}^{\mathrm{Dr},+,+}}$ is injective then:

(i) $\psi$ is injective (that is, $\mathcal{U}_{q}^{\mathrm{Dr}} \cong \mathcal{U}_{q}^{\mathrm{DJ}}$, see Remark 4.2(iv));

(ii) $\mathcal{U}_{q}^{\mathrm{Dr},-} \otimes_{\mathbb{C}(q)} \mathcal{U}_{q}^{\mathrm{Dr}, 0} \otimes_{\mathbb{C}(q)} \mathcal{U}_{q}^{\mathrm{Dr},+} \cong \mathcal{U}_{q}^{\mathrm{Dr}}$.

Proof. It is enough to consider the following commutative diagram for all $N \in \mathbb{N}$ (see Propositions 4.3 and 5.1 and Remarks 3.5(vii)\&(viii), 3.7 and 4.2(iii)):

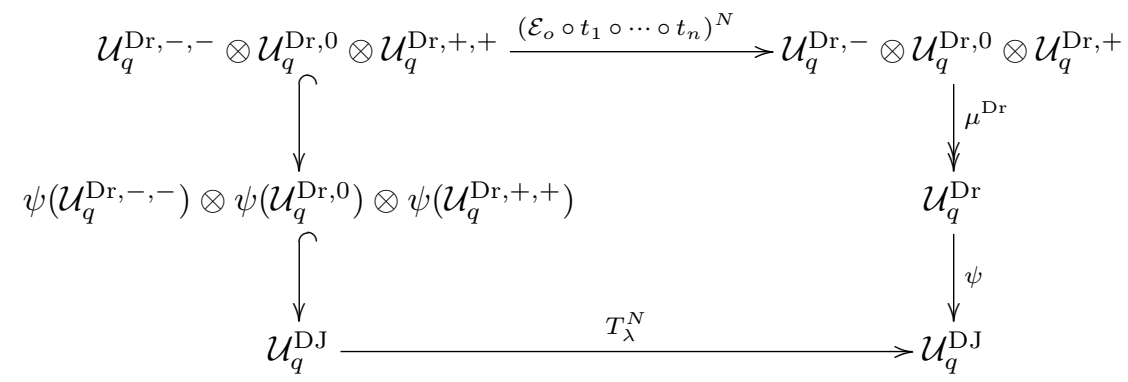

Remark 5.3. We have $\psi\left(\mathcal{U}_{q}^{\mathrm{Dr},+,+}\right) \subseteq \mathcal{U}_{q}^{\mathrm{DJ},+}$. On the other hand $\psi\left(\mathcal{U}_{q}^{\mathrm{Dr},+}\right) \nsubseteq$ $\mathcal{U}_{q}^{\mathrm{DJ},+}$. More precisely for all $i \in I_{0}$ and $r>0$ we have $\psi\left(X_{i,-r}^{+}\right) \in \tilde{\mathcal{U}}_{q}^{\text {DJ, }-}$ and $\psi\left(\mathcal{U}_{q}^{\mathrm{Dr},+}\right) \cap \tilde{\mathcal{U}}_{q,-r \delta+\alpha_{i}}^{\mathrm{DJ},-} \neq\{0\}$ if $\tilde{d}_{i} \mid r$.

In particular the Drinfeld triangular decomposition that we aim to prove will not correspond to the Drinfeld and Jimbo triangular decomposition, but will give rise to a substantially different decomposition. For a comparison between the two decompositions see Proposition 9.3.

Lemma 5.4. Let $\alpha \in Q_{0,+}, r \geq 0, i \in I_{0}$ be such that $r \delta+\alpha \in \Phi^{\mathrm{re}}$, or $(r \delta, i) \in$ $\hat{\Phi}^{\mathrm{im}}$. Then

(i) $E_{r \delta+\alpha} \in \psi\left(\mathcal{U}_{q}^{\mathrm{Dr},+}\right)$, and if $r>0$ then $F_{r \delta-\alpha} K_{r \delta-\alpha} \in \psi\left(\mathcal{U}_{q}^{\mathrm{Dr},+}\right)$;

(ii) $K_{\alpha-r \delta} E_{r \delta-\alpha} \in \psi\left(\mathcal{U}_{q}^{\mathrm{Dr},-}\right)$ if $r>0$;

(iii) $E_{(r \delta, i)} \in \psi\left(\mathcal{U}_{q}^{\mathrm{Dr}, 0}\right)$ if $r>0$.

Proof. Define

$$
U=\left\{x \in \mathcal{U}_{q}^{\mathrm{DJ}} \mid \forall N \gg 0, T_{\lambda}^{-N}(x) \in \mathcal{U}_{q}^{\mathrm{DJ},+}, T_{\lambda}^{N}(x) \in \tilde{\mathcal{U}}_{q}^{\mathrm{DJ},-}\right\} .
$$

Then: 
(a) $U$ is a $T_{\lambda}^{ \pm 1}$-stable $\mathbb{C}(q)$-subalgebra of $\mathcal{U}_{q}^{\text {DJ }}$ (obvious).

(b) $\psi\left(\mathcal{U}_{q}^{\mathrm{Dr},+,+}\right) \subseteq U$ thanks to Remarks 2.3 (vi), 3.5(vi) and 5.3.

(c) $\psi\left(\mathcal{U}_{q}^{\mathrm{Dr},+}\right) \subseteq U$ thanks to (a), (b) and Remark 3.5(vii).

(d) $U=\psi\left(\mathcal{U}_{q}^{\mathrm{Dr},+}\right)$ : consider the identifications induced by the product

$$
\begin{aligned}
\mathcal{U}_{q}^{\mathrm{DJ}} & \cong \psi\left(\mathcal{U}_{q}^{\mathrm{Dr},-}\right) \otimes \psi\left(\mathcal{U}_{q}^{\mathrm{Dr}, 0}\right) \otimes \psi\left(\mathcal{U}_{q}^{\mathrm{Dr},+}\right) \\
& \cong \psi\left(\mathcal{U}_{q}^{\mathrm{Dr},-}\right) \otimes \psi\left(\mathcal{U}_{q}^{\mathrm{Dr}, 0,-}\right) \otimes \psi\left(\mathcal{U}_{q}^{\mathrm{Dr}, 0,0}\right) \otimes \psi\left(\mathcal{U}_{q}^{\mathrm{Dr}, 0,+}\right) \otimes \psi\left(\mathcal{U}_{q}^{\mathrm{Dr},+}\right)
\end{aligned}
$$

and note that through these isomorphisms, for every $u \in \mathcal{U}_{q}^{\text {DJ }}$ there exists $\tilde{N} \in \mathbb{Z}$ such that for all $N>\tilde{N}$,

$$
T_{\lambda}^{-N}(u) \in \psi\left(\mathcal{U}_{q}^{\mathrm{Dr},-,-}\right) \otimes \psi\left(\mathcal{U}_{q}^{\mathrm{Dr}, 0,-}\right) \otimes \psi\left(\mathcal{U}_{q}^{\mathrm{Dr}, 0,0}\right) \otimes \psi\left(\mathcal{U}_{q}^{\mathrm{Dr}, 0,+}\right) \otimes \psi\left(\mathcal{U}_{q}^{\mathrm{Dr},+,+}\right) ;
$$

moreover

$$
\begin{gathered}
\psi\left(\mathcal{U}_{q}^{\mathrm{Dr},-,-}\right) \otimes \psi\left(\mathcal{U}_{q}^{\mathrm{Dr}, 0,-}\right) \subseteq \mathcal{U}_{q}^{\mathrm{DJ},-}, \quad \psi\left(\mathcal{U}_{q}^{\mathrm{Dr}, 0,0}\right) \subseteq \mathcal{U}_{q}^{\mathrm{DJ}, 0}, \\
\psi\left(\mathcal{U}_{q}^{\mathrm{Dr}, 0,+}\right) \otimes \psi\left(\mathcal{U}_{q}^{\mathrm{Dr},+,+}\right) \subseteq \mathcal{U}_{q}^{\mathrm{DJ},+} ;
\end{gathered}
$$

hence if $u \in U$ the condition $T_{\lambda}^{-N}(u) \in \mathcal{U}_{q}^{\mathrm{DJ},+}$ for all $N \gg 0$ and the triangular decomposition of $\mathcal{U}_{q}^{\mathrm{DJ}}$ imply that $u \in \psi\left(\mathcal{U}_{q}^{\mathrm{Dr}, 0,+}\right) \otimes \psi\left(\mathcal{U}_{q}^{\mathrm{Dr},+}\right)$; but then for all $N \gg 0$

$$
T_{\lambda}^{N}(u) \in \psi\left(\mathcal{U}_{q}^{\mathrm{Dr}, 0,+}\right) \otimes \tilde{\mathcal{U}}_{q}^{\mathrm{DJ},-},
$$

and again since $\psi\left(\mathcal{U}_{q}^{\mathrm{Dr}, 0,+}\right) \subseteq \mathcal{U}_{q}^{\mathrm{DJ},+}$, the condition $T_{\lambda}^{N}(u) \in \tilde{\mathcal{U}}_{q}^{\mathrm{DJ},-}$ for all $N \gg 0$ and the triangular decomposition of $\mathcal{U}_{q}^{\mathrm{DJ}}$ imply that $u \in \psi\left(\mathcal{U}_{q}^{\mathrm{Dr},+}\right)$, which implies the claim.

(e) $E_{r \delta+\alpha} \in U$ thanks to Remark 2.3(vi).

(f) $F_{r \delta-\alpha} K_{r \delta-\alpha} \in U$ thanks to (a) and (e), since $F_{r \delta-\alpha} K_{r \delta-\alpha}$ is $T_{\lambda}$-conjugate to any $E_{s \delta+\alpha}$ with $s \geq 0$ such that $\langle\lambda \mid \alpha\rangle \mid r+s$ (see Remark 2.3(vi)).

(d)-(f) imply (i). Applying $\Omega$ to (f) we get (ii), while (iii) is a straightforward consequence of the definitions.

Corollary 5.5. $\mathcal{U}_{q}^{\mathrm{DJ},+} \cap \psi\left(\mathcal{U}_{q}^{\mathrm{Dr},+}\right)$ is the $\mathbb{C}(q)$-linear span of the ordered monomials in the $E_{r \delta+\alpha}$ 's with $r \geq 0$ and $\alpha \in Q_{0,+}$ such that $r \delta+\alpha \in \Phi^{\mathrm{re}}$.

Proof. Let $U_{+}$be the $\mathbb{C}(q)$-linear span of the ordered monomials in the $E_{\beta_{r}}$ 's with $r \leq 0, U_{-}$be the $\mathbb{C}(q)$-linear span of the ordered monomials in the $E_{\beta_{r}}$ 's with $r \geq 1$, and $U_{0}$ be the $\mathbb{C}(q)$-linear span of the monomials in the positive imaginary root vectors. Then the PBW-basis of $\mathcal{U}_{q}^{\mathrm{DJ},+}$ says that $\mathcal{U}_{q}^{\mathrm{DJ},+} \cong U_{-} \otimes U_{0} \otimes U_{+}$. But

$$
U_{-} \otimes U_{0} \subseteq \psi\left(\mathcal{U}_{q}^{\mathrm{Dr},-}\right) \otimes \psi\left(\mathcal{U}_{q}^{\mathrm{Dr}, 0}\right), \quad U_{+} \subseteq \psi\left(\mathcal{U}_{q}^{\mathrm{Dr},+}\right)
$$


and

$$
\psi\left(\mathcal{U}_{q}^{\mathrm{Dr},-}\right) \otimes \psi\left(\mathcal{U}_{q}^{\mathrm{Dr}, 0}\right) \otimes \psi\left(\mathcal{U}_{q}^{\mathrm{Dr},+}\right) \cong \mathcal{U}_{q}^{\mathrm{DJ}},
$$

so that $\mathcal{U}_{q}^{\mathrm{DJ},+} \cap \psi\left(\mathcal{U}_{q}^{\mathrm{Dr},+}\right) \subseteq U_{+}$, which is the assertion, thanks to Lemma 5.4(i).

\section{$\S 6$. Integer form}

We are reduced to proving that $\left.\psi\right|_{\mathcal{U}_{q}^{\mathrm{Dr},+,+}}: \mathcal{U}_{q}^{\mathrm{Dr},+,+} \rightarrow \mathcal{U}_{q}^{\mathrm{DJ},+}$ is injective, and we want to show it through specialization at 1 . This requires passing to integer forms of $\mathcal{U}_{q}^{\mathrm{Dr},+,+}$ and $\mathcal{U}_{q}^{\mathrm{DJ},+}$ and to their presentations by generators and relations.

To this end we start with some notation, underlining that in this section we work with the $\operatorname{ring} \mathcal{A}=\mathbb{C}[q]_{(q-1)}$ (the localization of $\mathbb{C}[q]$ at $(q-1)$ ).

Notation 6.1. (i) $\mathcal{F}_{+}$is the $\mathcal{A}$-algebra freely generated by $\left\{X_{i, r}^{+} \mid i \in I_{0}, r \geq 0\right\}$;

(ii) $\mathcal{I}_{+}$is the ideal of $\mathcal{F}_{+}$defined by the relations $\left(Z X_{+}^{+}\right),\left(D R_{+}\right),\left(S_{+}\right)$and $\left(U 3_{+}\right)$ (see Notation 3.9);

(iii) $t_{+}^{\prime}: \mathcal{F}_{+} \rightarrow \mathcal{F}_{+}$is the $\mathcal{A}$-endomorphism defined by $X_{i, r}^{+} \mapsto o(i) X_{i, r+\tilde{d}_{i}}^{+}$(see Definitions 3.4(ii)\&(iii) and 4.1); we also denote by $\bar{t}_{+}^{\prime}$ the $\mathcal{A}$-endomorphism induced by $t_{+}^{\prime}$ on $\mathcal{F}_{+} / \mathcal{I}_{+}$.

Remark 6.2. (i) $\mathcal{F}_{+}, \mathcal{I}_{+}$and consequently also $\mathcal{F}_{+} / \mathcal{I}_{+}$are all $Q$-graded where the degree of $X_{i, r}^{+}$is $\alpha_{i}+r \delta$;

(ii) the $\mathcal{A}$-modules $\left(\mathcal{F}_{+}\right)_{\alpha}$ and $\left(\mathcal{F}_{+} / \mathcal{I}_{+}\right)_{\alpha}(\alpha \in Q)$ are finitely generated: they are generated over $\mathcal{A}$ by

$$
\left\{X_{i_{1}, m_{1}}^{+} \cdot \ldots \cdot X_{i_{h}, m_{h}}^{+} \mid i_{r} \in I_{0}, m_{r} \geq 0 \forall r=1, \ldots, h, \sum_{r=1}^{h} m_{r} \delta+\alpha_{i_{r}}=\alpha\right\} ;
$$

(iii) the natural map $f_{+}: \mathcal{F}_{+} / \mathcal{I}_{+} \rightarrow \mathcal{U}_{q}^{\text {Dr }}$ is well defined (see Definition 3.1 and Remark 3.10(i));

(iv) $f_{+} \circ \bar{t}_{+}^{\prime}=\mathcal{E}_{o} \circ t_{1}^{-1} \circ \ldots \circ t_{n}^{-1} \circ f_{+}$.

Remark 6.3. Of course $\mathbb{C}(q) \otimes_{\mathcal{A}} f_{+}\left(\mathcal{F}_{+} / \mathcal{I}_{+}\right) \cong \mathcal{U}_{q}^{\mathrm{Dr},+,+}$ and $f_{+}\left(\mathcal{F}_{+} / \mathcal{I}_{+}\right)$is an integer form of $\mathcal{U}_{q}^{\mathrm{Dr},+,+}$ : indeed, $f_{+}\left(\mathcal{F}_{+} / \mathcal{I}_{+}\right)$is a direct sum of finitely generated $\mathcal{A}$-submodules of a $\mathbb{C}(q)$-vector space, hence it is free over $\mathcal{A}$.

In particular a $\mathbb{C}(q)$-linear map defined on $\mathcal{U}_{q}^{\mathrm{Dr},+,+}$ is injective if and only if its restriction to $f_{+}\left(\mathcal{F}_{+} / \mathcal{I}_{+}\right)$is injective.

Corollary 6.4. If $\psi \circ f_{+}$is injective then:

(i) $f_{+}$is injective, hence $\mathcal{F}_{+} / \mathcal{I}_{+}$is an integer form of $\mathcal{U}_{q}^{\mathrm{Dr},+,+}$ (see Remark 6.3);

(ii) $\left.\psi\right|_{f_{+}\left(\mathcal{F}_{+} / \mathcal{I}_{+}\right)}$is injective (then so are $\left.\psi\right|_{\mathcal{U}_{q}^{\text {Dr, },+}}$ and $\psi$, see Proposition $5.2(\mathrm{i})$ and Remark 6.3). 
Remark 6.5. The image of $\psi \circ f_{+}$is contained in $\mathcal{U}_{\mathcal{A}}^{\mathrm{DJ},+}$. Indeed, $\psi\left(X_{i, r}^{+}\right) \in \mathcal{U}_{\mathcal{A}}^{\mathrm{DJ}} \cap$ $\mathcal{U}_{q}^{\mathrm{DJ},+}=\mathcal{U}_{\mathcal{A}}^{\mathrm{DJ},+}$ if $r \geq 0$ (see Definition 4.1 and Remarks 2.3(iii) and 2.4(iii)).

Notation 6.6. Set $\tilde{\psi}=\psi \circ f_{+}: \mathcal{F}_{+} / \mathcal{I}_{+} \rightarrow \mathcal{U}_{\mathcal{A}}^{\mathrm{DJ},+}$.

Remark 6.7. $\tilde{\psi}$ is obviously homogeneous, that is, $\tilde{\psi}=\bigoplus_{\alpha \in Q_{+}} \tilde{\psi}_{\alpha}$ with $\tilde{\psi}_{\alpha}=$ $\left.\tilde{\psi}\right|_{\left(\mathcal{F}_{+} / \mathcal{I}_{+}\right)_{\alpha}}$ and consequently $\tilde{\psi}_{1}=\bigoplus_{\alpha \in Q_{+}}\left(\tilde{\psi}_{\alpha}\right)_{1}$ where $\left(\tilde{\psi}_{\alpha}\right)_{1}$ is the specialization at 1 of $\tilde{\psi}_{\alpha}$.

Since $\left(\mathcal{F}_{+} / \mathcal{I}_{+}\right)_{\alpha}$ is finitely generated over $\mathcal{A}$ and $\mathcal{U}_{\mathcal{A}, \alpha}^{\mathrm{DJ},+}$ is free over $\mathcal{A}$, we infer that for each $\alpha \in Q_{+}, \tilde{\psi}_{\alpha}$ is injective if $\left(\tilde{\psi}_{\alpha}\right)_{1}$ is injective (see Proposition 0.1).

Thus $\tilde{\psi}$ is injective if $\tilde{\psi}_{1}$ is injective.

\section{$\S 7$. Specialization at $q=1$}

We are reduced to studying the specialization at 1 of $\tilde{\psi}$. To this end it is important that we first understand the structure of the specialization at 1 of $\mathcal{F}_{+} / \mathcal{I}_{+}$and of $\mathcal{U}_{\mathcal{A}}^{\mathrm{DJ},+}$. Since, as recalled in Remark 7.1 below, the specialization at 1 of $\mathcal{U}_{\mathcal{A}}^{\mathrm{DJ},+}$ is well known, we concentrate on the description of the specialization of $\mathcal{F}_{+} / \mathcal{I}_{+}$.

Of course a first presentation by generators and relations of the specialization at 1 of $\mathcal{F}_{+} / \mathcal{I}_{+}$is immediate, by just specializing at 1 the defining relations of $\mathcal{F}_{+} / \mathcal{I}_{+}$(see Proposition 7.2 ). The present section is devoted to simplifying these specialized relations.

Remark 7.1. Thanks to Remark 2.4(iv), the specialization at 1 of $\mathcal{U}_{\mathcal{A}}^{\mathrm{DJ},+}$ is the enveloping algebra of the Lie algebra generated by $\left\{e_{i} \mid i \in I\right\}$ with relations $\left(\operatorname{ad} e_{i}\right)^{1-a_{i j}}\left(e_{j}\right)=0$ when $i \neq j$ (Serre relations), which is well known to be the positive part of the Kac-Moody algebra $\hat{\mathfrak{g}}=\hat{\mathfrak{g}}\left(X_{\tilde{n}}^{(k)}\right)$ and also of the loop algebra $\left(\mathfrak{g} \otimes_{\mathbb{C}} \mathbb{C}\left[t^{ \pm 1}\right]\right)^{\chi} \supseteq \mathfrak{g}^{\chi}=\mathfrak{g}_{0}($ see $[\mathrm{K}])$.

By the very definition of $\mathcal{F}_{+}$and $\mathcal{I}_{+}$we have

Proposition 7.2. The specialization at 1 of $\mathcal{F}_{+} / \mathcal{I}_{+}$is the (associative) algebra generated by $\left\{x_{i, r}^{+} \mid i \in I_{0}, r \geq 0\right\}$ with the following relations $\left(\tilde{d} r_{+}\right)$:

$(z x) \quad x_{i, r}^{+}=0 \quad\left(\tilde{d}_{i} \nmid r\right)$,

$(x d) \quad\left[x_{i, r+\tilde{d}_{i j}}^{+}, x_{j, s}^{+}\right]+\left[x_{j, s+\tilde{d}_{i j}}^{+}, x_{i, r}^{+}\right]=0 \quad\left((i, r),(j, s) \in I_{\mathbb{Z}}, a_{i j}<0\right)$,

$(x 1) \quad \sum_{\sigma \in \mathcal{S}_{2}} \sigma \cdot\left[x_{i, r_{1}+\tilde{d}_{i}}^{+}, x_{i, r_{2}}^{+}\right]=0 \quad\left(\left(r_{1}, r_{2}\right) \in \mathbb{N}^{2},\left(X_{\tilde{n}}^{(k)}, d_{i}\right) \neq\left(A_{2 n}^{(2)}, 1\right)\right)$

$(x 2) \quad \sum_{\sigma \in \mathcal{S}_{2}} \sigma \cdot\left[x_{i, r_{1}+2}^{+}, x_{i, r_{2}}^{+}\right]=0 \quad\left(\left(r_{1}, r_{2}\right) \in \mathbb{N}^{2},\left(X_{\tilde{n}}^{(k)}, d_{i}\right)=\left(A_{2 n}^{(2)}, 1\right)\right)$, 
$(x 3)$

$$
\begin{aligned}
\sum_{\sigma \in \mathcal{S}_{3}} \sigma \cdot\left[\left[x_{i, r_{1}+1}^{+}, x_{i, r_{2}}^{+}\right], x_{i, r_{3}}^{+}\right] & =0 \\
& \left(\left(r_{1}, r_{2}, r_{3}\right), \in \mathbb{N}^{3},\left(X_{\tilde{n}}^{(k)}, d_{i}\right)=\left(A_{2 n}^{(2)}, 1\right)\right),
\end{aligned}
$$

(t2) $\quad \sum_{\sigma \in \mathcal{S}_{2}} \sigma \cdot\left[\left[x_{j, s}^{+}, x_{i, r_{1}+1}^{+}\right], x_{i, r_{2}}^{+}\right]=0$,

$$
\left(i, j \in I_{0}, a_{i j}=-2, k=2, X_{\tilde{n}}^{(k)} \neq A_{2 n}^{(2)},\left(r_{1}, r_{2}\right) \in \mathbb{N}^{2}, s \in \mathbb{Z}\right),
$$

$$
\begin{aligned}
\sum_{\sigma \in \mathcal{S}_{2}} \sigma \cdot\left(\left[\left[x_{j, s}^{+}, x_{i, r_{1}+1}^{+}\right], x_{i, r_{2}}^{+}\right]+\left[\left[x_{j, s}^{+}, x_{i, r_{2}}^{+}\right], x_{i, r_{1}+1}^{+}\right]\right)=0 \\
\left(i, j \in I_{0}, a_{i j}=-2, X_{\tilde{n}}^{(k)}=A_{2 n}^{(2)},\left(r_{1}, r_{2}\right) \in \mathbb{N}^{2}, s \in \mathbb{Z}\right),
\end{aligned}
$$

$\sum_{\sigma \in \mathcal{S}_{2}} \sigma \cdot\left(2\left[\left[x_{j, s}^{+}, x_{i, r_{1}+2}^{+}\right], x_{i, r_{2}}^{+}\right]+\left[\left[x_{j, s}^{+}, x_{i, r_{1}+1}^{+}\right], x_{i, r_{2}+1}^{+}\right]\right)=0$

$$
\left(i, j \in I_{0}, a_{i j}=-3, k=3,\left(r_{1}, r_{2}\right) \in \mathbb{N}^{2}, s \in \mathbb{Z}\right),
$$

$\sum_{\sigma \in \mathcal{S}_{3}} \sigma \cdot\left[\left[\left[x_{j, s}^{+}, x_{i, r_{1}+1}^{+}\right], x_{i, r_{2}}^{+}\right], x_{i, r_{3}}^{+}\right]=0$

$$
\left(i, j \in I_{0}, a_{i j}=-3, k=3,\left(r_{1}, r_{2}, r_{3}\right) \in \mathbb{N}^{3}, s \in \mathbb{Z}\right),
$$

$(s)$

$$
\begin{aligned}
& \sum_{\sigma \in \mathcal{S}_{1-a_{i j}}} \sigma \cdot\left[\ldots\left[\left[x_{j, s}^{+}, x_{i, r_{1}}^{+}\right], x_{i, r_{2}}^{+}\right], \ldots, x_{i, r_{1-a_{i j}}}^{+}\right]=0 \\
&\left(i \neq j \in I_{0}, r \in \mathbb{N}^{1-a_{i j}}, s \in \mathbb{Z}\right) .
\end{aligned}
$$

Proof. All the relations $\left(\tilde{d} r_{+}\right)$are the immediate specialization at 1 of the relations $\left(Z X_{+}^{+}, D R_{+}, U 3_{+}, S_{+}\right)$, recalling Notation 1.5 and Remark 3.10(ii), and noticing that relations $\left(S 2_{+}\right)$specialize to

$$
\begin{aligned}
0 & =\sum_{\sigma \in \mathcal{S}_{2}}\left(2\left[\left[x_{j, s}^{+}, x_{i, r_{1}+1}^{+}\right], x_{i, r_{2}}^{+}\right]+\left[\left[x_{i, r_{1}+1}^{+}, x_{i, r_{2}}^{+}\right], x_{j, s}^{+}\right]\right) \\
& =\sum_{\sigma \in \mathcal{S}_{2}}\left(2\left[\left[x_{j, s}^{+}, x_{i, r_{1}+1}^{+}\right], x_{i, r_{2}}^{+}\right]+\left[x_{i, r_{1}+1}^{+},\left[x_{i, r_{2}}^{+}, x_{j, s}^{+}\right]\right]-\left[x_{i, r_{2}}^{+},\left[x_{i, r_{1}+1}^{+}, x_{j, s}^{+}\right]\right]\right) \\
& =\sum_{\sigma \in \mathcal{S}_{2}}\left(\left[\left[x_{j, s}^{+}, x_{i, r_{1}+1}^{+}\right], x_{i, r_{2}}^{+}\right]+\left[x_{i, r_{1}+1}^{+},\left[x_{i, r_{2}}^{+}, x_{j, s}^{+}\right]\right]\right),
\end{aligned}
$$

which is $(s 2)$.

Remark 7.3. In the relations $\left(\tilde{d} r_{+}\right)$(see Proposition 7.2) all the products are expressed in terms of brackets; hence the associative algebra generated by $\left\{x_{i, r}^{+} \mid\right.$ $\left.i \in I_{0}, r \geq 0\right\}$ with the relations $\left(\tilde{d} r_{+}\right)$is the enveloping algebra of the Lie algebra generated by $\left\{x_{i, r}^{+} \mid i \in I_{0}, r \geq 0\right\}$ with the relations $\left(\tilde{d} r_{+}\right)$. This Lie algebra plays a central role in the following.

Definition 7.4. $L_{+}$is the Lie algebra generated by $\left\{x_{i, r}^{+} \mid i \in I_{0}, r \geq 0\right\}$ with the relations $\left(\tilde{d} r_{+}\right)$. 
Remark 7.5. The specialization at 1 of $\mathcal{F}_{+} / \mathcal{I}_{+}$is the enveloping algebra $\mathcal{U}\left(L_{+}\right)$ of the Lie algebra $L_{+}$(see Proposition 7.2, Remark 7.3 and Definition 7.4). In particular $\tilde{\psi}_{1}$ is a homomorphism of associative algebras from $\mathcal{U}\left(L_{+}\right)$to $\mathcal{U}\left(\hat{\mathfrak{g}}^{+}\right)$ (see Remark 7.1). The next step is to prove that $\tilde{\psi}_{1}\left(L_{+}\right) \subseteq \hat{\mathfrak{g}}^{+}$, which implies that $\left.\tilde{\psi}_{1}\right|_{L_{+}}$is a Lie algebra homomorphism from $L_{+}$to $\hat{\mathfrak{g}}^{+}$and $\tilde{\psi}_{1}=\mathcal{U}\left(\left.\tilde{\psi}_{1}\right|_{L_{+}}\right)$.

Remark 7.6. We have $\tilde{\psi}_{1}\left(L_{+}\right) \subseteq\left(\mathfrak{g}_{+} \otimes_{\mathbb{C}} \mathbb{C}[t]\right)^{\chi} \subseteq \hat{\mathfrak{g}}^{+}$; in particular, thanks to Remark 7.5 we deduce that $\tilde{\psi}_{1}$ is injective if and only if $\left.\tilde{\psi}_{1}\right|_{L_{+}}$is injective.

Proof. Since $\tilde{\psi} \circ \bar{t}_{+}^{\prime}=T_{\lambda}^{-1} \circ \tilde{\psi}$ (see Remarks 4.2(iii) and 6.2(iv)), the claim follows from the fact that $\tilde{\psi}\left(x_{i, 0}^{+}\right)=e_{i} \in \mathfrak{g}_{0,+} \subseteq \hat{\mathfrak{g}}$, from Remarks 2.4 (viii) and 3.5(ix), and from the fact that $\hat{\mathfrak{g}}_{r \delta+\alpha_{i}} \subseteq\left(\mathfrak{g}_{+} \otimes \mathbb{C}[t]\right)^{\chi}$ for $i \in I_{0}$ and $r \geq 0$.

Proposition 7.7. $\left.\tilde{\psi}_{1}\right|_{L_{+}}: L_{+} \rightarrow\left(\mathfrak{g}_{+} \otimes_{\mathbb{C}} \mathbb{C}[t]\right)^{\chi}$ is surjective.

Proof. We have

$$
\left(\mathfrak{g}_{+} \otimes_{\mathbb{C}} \mathbb{C}[t]\right)^{\chi}=\bigoplus_{r \in \mathbb{N}}\left(\mathfrak{g}_{+}^{[r]} \otimes_{\mathbb{C}} \mathbb{C} t^{r}\right) \subseteq \bigoplus_{r \in \mathbb{N}}\left(\mathfrak{g}^{[r]} \otimes_{\mathbb{C}} \mathbb{C} t^{r}\right)=\left(\mathfrak{g} \otimes_{\mathbb{C}} \mathbb{C}[t]\right)^{\chi}
$$

where $\mathfrak{g}^{[r]}=\mathfrak{g}_{-}^{[r]} \oplus \mathfrak{h}^{[r]} \oplus \mathfrak{g}_{+}^{[r]}$ is well known to be a simple finite-dimensional $\mathfrak{g}_{0}=\mathfrak{g}^{[0]}$-module, hence a lowest weight cyclic $\mathfrak{g}_{+}^{[0]}=\mathfrak{g}_{0,+}$-module (see $[\mathrm{K}]$ ). Then $\mathfrak{g}_{+}^{[r]}\left(=\bigoplus_{\alpha \in Q_{0,+} \backslash\{0\}}\left(\mathfrak{g}^{[r]}\right)_{\alpha}\right)$ is generated as a $\mathfrak{g}_{0,+}$-module by

$$
\bigoplus_{i \in I_{0}}\left(\mathfrak{g}^{[r]}\right)_{\alpha_{i}}=\bigoplus_{i \in I_{0}}\left(\mathfrak{g}_{+}^{[r]}\right)_{\alpha_{i}} \quad\left(=\bigoplus_{\substack{i \in I_{0}: \\ \tilde{d}_{i} \mid r}}\left(\mathfrak{g}_{+}^{[r]}\right)_{\alpha_{i}} \text { since }\left(\mathfrak{g}_{+}^{[r]}\right)_{\alpha_{i}}=(0) \text { if } \tilde{d}_{i} \nmid r\right)
$$

that is, $\left(\mathfrak{g}_{+} \otimes_{\mathbb{C}} \mathbb{C}[t]\right)^{\chi}$ is generated as a $\mathfrak{g}_{0,+}$-module by $\bigoplus_{i \in I_{0}, r \in \mathbb{N}:}\left(\mathfrak{g}_{+}^{[r]}\right)_{\alpha_{i}} \otimes \mathbb{C} t^{r}$ or equivalently by $\left\{\tilde{\psi}_{1}\left(x_{i, r}^{+}\right) \mid i \in I_{0}, r \in \mathbb{N}\right.$ such that $\left.\tilde{d}_{i} \mid r\right\}$ since for all $i \in I_{0}$ and $r \in \mathbb{N}, \tilde{\psi}_{1}\left(x_{i, \tilde{d}_{i} r}^{+}\right)= \pm \tilde{T}_{\lambda}^{-r}\left(e_{i}\right) \neq 0$ (see Remarks 2.4(viii), 3.5(ix), 4.2(iii) and $6.2(\mathrm{iv}))$, and $\left(\mathfrak{g}_{+}^{\left[\tilde{d}_{i} r\right]}\right)_{\alpha_{i}}$ is one-dimensional.

This forces $\left\{\tilde{\psi}_{1}\left(x_{i, r}^{+}\right) \mid i \in I_{0}, r \in \mathbb{N}\right\}$, which obviously contains $\left\{e_{i}=\right.$ $\left.\tilde{\psi}_{1}\left(x_{i, 0}^{+}\right) \mid i \in I_{0}\right\}$, to generate $\left(\mathfrak{g}_{+} \otimes_{\mathbb{C}} \mathbb{C}[t]\right)^{\chi}$ also as a Lie algebra; the assertion follows.

Corollary 7.8. (i) $E_{r \delta+\alpha} \in \psi\left(\mathcal{U}_{q}^{\mathrm{Dr},+,+}\right)$ if $r \geq 0$ and $\alpha \in Q_{0,+} \backslash\{0\}$; (ii) $\mathcal{U}_{q}^{\mathrm{DJ},+} \cap \psi\left(\mathcal{U}_{q}^{\mathrm{Dr},+}\right)=\psi\left(\mathcal{U}_{q}^{\mathrm{Dr},+,+}\right)$.

Proof. (i) follows from (ii) by Corollary 5.5 (indeed, (i) and (ii) are equivalent claims because $\left.\psi\left(\mathcal{U}_{q}^{\mathrm{Dr},+,+}\right) \subseteq \mathcal{U}_{q}^{\mathrm{DJ},+} \cap \psi\left(\mathcal{U}_{q}^{\mathrm{Dr},+}\right)\right)$. So it is enough to compare the dimensions of the homogeneous components of $\mathcal{U}_{q}^{\mathrm{DJ},+} \cap \psi\left(\mathcal{U}_{q}^{\mathrm{Dr},+}\right)$ and $\psi\left(\mathcal{U}_{q}^{\mathrm{Dr},+,+}\right)$ : for all $\alpha \in Q$, 


$$
\begin{aligned}
\operatorname{dim}_{\mathbb{C}(q)} \mathcal{U}_{q, \alpha}^{\mathrm{DJ},+} \cap \psi\left(\mathcal{U}_{q}^{\mathrm{Dr},+}\right) & \geq \operatorname{dim}_{\mathbb{C}(q)} \psi\left(\mathcal{U}_{q, \alpha}^{\mathrm{Dr},+,+}\right)=\operatorname{rk}_{\mathcal{A}} \tilde{\psi}\left(\mathcal{F}_{+} / \mathcal{I}_{+}\right)_{\alpha} \\
=\operatorname{dim}_{\mathbb{C}} \tilde{\psi}_{1}\left(\mathcal{U}\left(L_{+}\right)_{\alpha}\right) & =\operatorname{dim}_{\mathbb{C}} \mathcal{U}\left(\left(\mathfrak{g}_{+} \otimes_{\mathbb{C}} \mathbb{C}[t]\right)^{\chi}\right)_{\alpha}=\operatorname{dim}_{\mathbb{C}(q)} \mathcal{U}_{q, \alpha}^{\mathrm{DJ},+} \cap \psi\left(\mathcal{U}_{q}^{\mathrm{Dr},+}\right)
\end{aligned}
$$

where the last two equalities follow respectively from Proposition 7.7 and from the comparison of the $\mathbb{C}(q)$-basis of $\mathcal{U}_{q}^{\mathrm{DJ},+} \cap \psi\left(\mathcal{U}_{q}^{\mathrm{Dr},+}\right)$ described in Corollary 5.5 with the PBW-basis of $\mathcal{U}\left(\left(\mathfrak{g}_{+} \otimes_{\mathbb{C}} \mathbb{C}[t]\right)^{\chi}\right)$.

Before proving, in $\S 8$, that the Lie-algebra homomorphism $\left.\tilde{\psi}_{1}\right|_{L_{+}}$is actually injective, in the remaining part of this section we simplify the relations defining $L_{+}$ (see the following computations, summarized in Corollary 7.29).

Remark 7.9. Relations $(x d)$ are equivalent to saying that if $a_{i j}<0, \tilde{d}_{i} \mid r$ and $\tilde{d}_{j} \mid s(i \neq j$ fixed $)$ then $\left[x_{i, r}^{+}, x_{j, s}^{+}\right]$depends only on $r+s$. Together with $(s)$ in case $a_{i j}=0$ they imply

$\left(x_{d}\right) \quad\left[x_{i, r}^{+}, x_{j, s}^{+}\right]$depends only on $r+s \quad\left(i \neq j \in I_{0}\right.$ fixed, $\left.\tilde{d}_{i}\left|r, \tilde{d}_{j}\right| s\right)$.

Lemma 7.10. Relations $(x 1)$ and $(x 2)$ are equivalent to

$\left(x_{1,2}\right) \quad\left[x_{i, r}^{+}, x_{i, s}^{+}\right]= \begin{cases}0 & \text { if }\left(X_{\tilde{n}}^{(k)}, d_{i}\right) \neq\left(A_{2 n}^{(2)}, 1\right) \text { or } 2 \mid r+s, \\ (-1)^{h}\left[x_{i, s+h+1}^{+}, x_{i, s+h}^{+}\right] & \text {if } r=s+2 h+1 ;\end{cases}$

in particular $(-1)^{s}\left[x_{i, r}^{+}, x_{i, s}^{+}\right]$depends only on $r+s$.

Proof. That $\left(x_{1,2}\right)$ implies $(x 1)$ and $(x 2)$ is obvious. Conversely:

Case $\left(X_{\tilde{n}}^{(k)}, d_{i}\right) \neq\left(A_{2 n}^{(2)}, 1\right)$ : of course we can suppose $r \geq s$ and proceed by induction on $r-s$, the cases $r=s$ and $r=s+\tilde{d}_{i}$ being obvious; if $r>s+\tilde{d}_{i}$ then

$$
\left[x_{i, r}^{+}, x_{i, s}^{+}\right]=-\left[x_{i, s+\tilde{d}_{i}}^{+}, x_{i, r-\tilde{d}_{i}}^{+}\right]=\left[x_{i, r-\tilde{d}_{i}}^{+}, x_{i, s+\tilde{d}_{i}}^{+}\right]=0 \quad\left(r-\tilde{d}_{i} \geq s+\tilde{d}_{i}\right) .
$$

Case $\left(X_{\tilde{n}}^{(k)}, d_{i}\right)=\left(A_{2 n}^{(2)}, 1\right)$ : again we can suppose $r \geq s$ and proceed by induction on $r-s$, the cases $r-s=0,1,2$ being obvious:

$$
\begin{aligned}
& r-s=3 \Rightarrow\left[x_{i, r}^{+}, x_{i, s}^{+}\right]=\left[x_{i, s+3}^{+}, x_{i, s}^{+}\right]=-\left[x_{i, s+2}^{+}, x_{i, s+1}^{+}\right] ; \\
& r-s>3 \Rightarrow\left[x_{i, r}^{+}, x_{i, s}^{+}\right]=-\left[x_{i, s+2}^{+}, x_{i, r-2}^{+}\right]=\left[x_{i, r-2}^{+}, x_{i, s+2}^{+}\right],
\end{aligned}
$$

from which the claim follows by the inductive hypothesis, since $r-2 \geq s+2$.

Corollary 7.11. If $\left(X_{\tilde{n}}^{(k)}, d_{i}\right) \neq\left(A_{2 n}^{(2)}, 1\right)$ or $2 \mid r+s$ we have $\left[\left[a, x_{i, r}^{+}\right], x_{i, s}^{+}\right]=$ $\left[\left[a, x_{i, s}^{+}\right], x_{i, r}^{+}\right]$for all $a \in L_{+}$.

Proof. Indeed, $\left[\left[a, x_{i, r}^{+}\right], x_{i, s}^{+}\right]-\left[\left[a, x_{i, s}^{+}\right], x_{i, r}^{+}\right]=\left[a,\left[x_{i, r}^{+}, x_{i, s}^{+}\right]\right]=0$ thanks to Lemma 7.10 .

Lemma 7.12. If $\left(X_{\tilde{n}}^{(k)}, d_{i}\right)=\left(A_{2 n}^{(2)}, 1\right)$ relations $\left(x_{1,2}\right),(x 3)$ imply

$$
\left[\left[x_{i, r_{1}}^{+}, x_{i, r_{2}}^{+}\right], x_{i, r_{3}}^{+}\right]=0 \quad\left(\left(r_{1}, r_{2}, r_{3}\right) \in \mathbb{N}^{3}\right) .
$$


Proof. Thanks to Lemma 7.10 it is enough to prove that

$$
\left[\left[x_{i, r+1}^{+}, x_{i, r}^{+}\right], x_{i, s}^{+}\right]=0 \quad \forall r, s \in \mathbb{N} .
$$

Recall that by $(x 3)$,

$$
\left[\left[x_{i, r+1}^{+}, x_{i, r}^{+}\right], x_{i, s}^{+}\right]+\left[\left[x_{i, r+1}^{+}, x_{i, s}^{+}\right], x_{i, r}^{+}\right]+\left[\left[x_{i, s+1}^{+}, x_{i, r}^{+}\right], x_{i, r}^{+}\right]=0
$$

if $r+s+1$ is even then, by $\left(x_{1,2}\right)$,

$$
\left[x_{i, r+1}^{+}, x_{i, s}^{+}\right]=0=\left[x_{i, s+1}^{+}, x_{i, r}^{+}\right],
$$

so that

$$
\left[\left[x_{i, r+1}^{+}, x_{i, r}^{+}\right], x_{i, s}^{+}\right]=0 ;
$$

if $r+s$ is even then by Corollary 7.11,

$$
\left[\left[x_{i, r+1}^{+}, x_{i, r}^{+}\right], x_{i, s}^{+}\right]=\left[\left[x_{i, r+1}^{+}, x_{i, s}^{+}\right], x_{i, r}^{+}\right]
$$

moreover by $\left(x_{1,2}\right),\left[x_{i, s+1}^{+}, x_{i, r}^{+}\right]= \pm\left[x_{i, r+1}^{+}, x_{i, s}^{+}\right]$, so that

$$
\begin{aligned}
0 & =\left[\left[x_{i, r+1}^{+}, x_{i, r}^{+}\right], x_{i, s}^{+}\right]+\left[\left[x_{i, r+1}^{+}, x_{i, s}^{+}\right], x_{i, r}^{+}\right]+\left[\left[x_{i, s+1}^{+}, x_{i, r}^{+}\right], x_{i, r}^{+}\right] \\
& =(2 \pm 1))\left[\left[x_{i, r+1}^{+}, x_{i, s}^{+}\right], x_{i, r}^{+}\right],
\end{aligned}
$$

which is $\left[\left[x_{i, r+1}^{+}, x_{i, r}^{+}\right], x_{i, s}^{+}\right]=0$.

Proposition 7.13. Relations $(x 1),(x 2)$, (x3) are equivalent to relations $\left(x_{1,2}\right)$, $\left(x_{3}\right)$ (it is obvious that $\left(x_{3}\right)$ implies $(x 3)$ ).

Lemma 7.14. Let $i, j \in I_{0}$ and $r_{1}, r_{2}, s \in \mathbb{Z}$ be such that $a_{i j}<0, \tilde{d}_{i} \mid r_{1}, r_{2}$ and $\tilde{d}_{j} \mid s$. Then:

(i) if $\tilde{d}_{i} \geq \tilde{d}_{j}$ and $\left(X_{\tilde{n}}^{(k)}, d_{i}\right) \neq\left(A_{2 n}^{(2)}, 1\right)$ then

$$
\left[\left[x_{j, s}^{+}, x_{i, r_{1}}^{+}\right], x_{i, r_{2}}^{+}\right]=\left[\left[x_{j, s+r_{1}+r_{2}}^{+}, x_{i, 0}^{+}\right], x_{i, 0}^{+}\right] ;
$$

(ii) if $1=\tilde{d}_{i}<\tilde{d}_{j}=k$ or $\left(X_{\tilde{n}}^{(k)}, d_{i}\right)=\left(A_{2 n}^{(2)}, 1\right)$, and $k \mid r_{2}-\varepsilon_{2}\left(0 \leq \varepsilon_{2}<k\right)$ then $\left[\left[x_{j, s}^{+}, x_{i, r_{1}}^{+}\right], x_{i, r_{2}}^{+}\right]$depends only on $\left(s+r_{1}+r_{2}, \varepsilon_{2}\right)$.

Proof. (i) is an immediate consequence of relations $\left(x_{d}\right),\left(x_{1,2}\right)$ and of Corollary 7.11:

$$
\begin{aligned}
{\left[\left[x_{j, s}^{+}, x_{i, r_{1}}^{+}\right], x_{i, r_{2}}^{+}\right] } & =\left[\left[x_{j, s+r_{1}}^{+}, x_{i, 0}^{+}\right], x_{i, r_{2}}^{+}\right]=\left[\left[x_{j, s+r_{1}}^{+}, x_{i, r_{2}}^{+}\right], x_{i, 0}^{+}\right] \\
& =\left[\left[x_{j, s+r_{1}+r_{2}}^{+}, x_{i, 0}^{+}\right], x_{i, 0}^{+}\right]
\end{aligned}
$$


(ii) is similar: if $\tilde{d}_{i}<\tilde{d}_{j}$ or $2 \mid r_{1}+r_{2}$, then

$$
\begin{aligned}
{\left[\left[x_{j, s}^{+}, x_{i, r_{1}}^{+}\right], x_{i, r_{2}}^{+}\right] } & =\left[\left[x_{j, s}^{+}, x_{i, r_{2}}^{+}\right], x_{i, r_{1}}^{+}\right]=\left[\left[x_{j, s+r_{2}-\varepsilon_{2}}^{+}, x_{i, \varepsilon_{2}}^{+}\right], x_{i, r_{1}}^{+}\right] \\
& =\left[\left[x_{j, s+r_{2}-\varepsilon_{2}}^{+}, x_{i, r_{1}}^{+}\right], x_{i, \varepsilon_{2}}^{+}\right]
\end{aligned}
$$

if $\left(X_{\tilde{n}}^{(k)}, d_{i}\right)=\left(A_{2 n}^{(2)}, 1\right)$, and $s+r_{1}>0$ or $2 \mid r_{2}$, then

$$
\left[\left[x_{j, s}^{+}, x_{i, r_{1}}^{+}\right], x_{i, r_{2}}^{+}\right]=\left[\left[x_{j, s+r_{1}-\varepsilon_{2}}^{+}, x_{i, \varepsilon_{2}}^{+}\right], x_{i, r_{2}}^{+}\right]=\left[\left[x_{j, s+r_{1}-\varepsilon_{2}}^{+}, x_{i, r_{2}}^{+}\right], x_{i, \varepsilon_{2}}^{+}\right] \text {; }
$$

in both cases $\left[\left[x_{j, s}^{+}, x_{i, r_{1}}^{+}\right], x_{i, r_{2}}^{+}\right]$depends only on $\left(s+r_{1}+r_{2}-\varepsilon_{2}, \varepsilon_{2}\right)$, that is, on $\left(s+r_{1}+r_{2}, \varepsilon_{2}\right)$; finally if $\left(X_{\tilde{n}}^{(k)}, d_{i}\right)=\left(A_{2 n}^{(2)}, 1\right)$ and $s=r_{1}=0, r_{2}=2 r+1$ we can suppose $r>0$ and we have

$$
\begin{aligned}
{\left[\left[x_{j, 0}^{+}, x_{i, 0}^{+}\right], x_{i, 2 r+1}^{+}\right] } & =\left[\left[x_{j, 0}^{+}, x_{i, 2 r+1}^{+}\right], x_{i, 0}^{+}\right]+\left[x_{j, 0}^{+},\left[x_{i, 0}^{+}, x_{i, 2 r+1}^{+}\right]\right] \\
& =\left[\left[x_{j, 0}^{+}, x_{i, 2 r+1}^{+}\right], x_{i, 0}^{+}\right]+\left[x_{j, 0}^{+},\left[x_{i, 2}^{+}, x_{i, 2 r-1}^{+}\right]\right] \\
& =\left[\left[x_{j, 0}^{+}, x_{i, 2 r+1}^{+}\right], x_{i, 0}^{+}\right]+\left[\left[x_{j, 0}^{+}, x_{i, 2}^{+}\right], x_{i, 2 r-1}^{+}\right]-\left[\left[x_{j, 0}^{+}, x_{i, 2 r-1}^{+}\right], x_{i, 2}^{+}\right] \\
& =\left[\left[x_{j, 0}^{+}, x_{i, 2}^{+}\right], x_{i, 2 r-1}^{+}\right]
\end{aligned}
$$

and the claim follows from the previous cases.

Proposition 7.15. Relations $\left(x_{d}\right),\left(x_{1,2}\right)$ and $(t 2)$ are equivalent to relations $\left(x_{d}\right)$, $\left(x_{1,2}\right),\left(t_{2}^{\prime}\right)$ and $\left(t_{2}^{\prime \prime}\right)$, where

$$
\begin{gathered}
{\left[\left[x_{j, s}^{+}, x_{i, 1}^{+}\right], x_{i, 0}^{+}\right]=0,} \\
{\left[\left[x_{j, s}^{+}, x_{i, 1}^{+}\right], x_{i, 1}^{+}\right]=-\left[\left[x_{j, s+2}^{+}, x_{i, 0}^{+}\right], x_{i, 0}^{+}\right]}
\end{gathered}
$$

$\left(k=2, a_{i j}=-2, X_{\tilde{n}}^{(k)} \neq A_{2 n}^{(2)}\right)$.

Proof. Indeed, Corollary 7.11 and Remark 7.14 imply that

$$
\begin{aligned}
\sum_{\sigma \in \mathcal{S}_{2}} \sigma \cdot\left[\left[x_{j, s}^{+}, x_{i, r_{1}+1}^{+}\right], x_{i, r_{2}}^{+}\right]=\left[\left[x_{j, s}^{+}, x_{i, r_{1}+1}^{+}\right], x_{i, r_{2}}^{+}\right]+\left[\left[x_{j, s}^{+}, x_{i, r_{1}}^{+}\right], x_{i, r_{2}+1}^{+}\right] \\
= \begin{cases}{\left[\left[x_{j, s+r_{1}+r_{2}}^{+}, x_{i, 1}^{+}\right], x_{i, 0}^{+}\right]+\left[\left[x_{j, s+r_{1}+r_{2}}^{+}, x_{i, 0}^{+}\right], x_{i, 1}^{+}\right]} & \text {if } 2 \mid r_{1}+r_{2}, \\
{\left[\left[x_{j, s+r_{1}+r_{2}-1}^{+}, x_{i, 1}^{+}\right], x_{i, 1}^{+}\right]+\left[\left[x_{j, s+r_{1}+r_{2}+1}^{+}, x_{i, 0}^{+}\right], x_{i, 0}^{+}\right]} & \text {otherwise }\end{cases}
\end{aligned}
$$

but by Corollary 7.11 we have

$$
\left[\left[x_{j, s+r_{1}+r_{2}}^{+}, x_{i, 1}^{+}\right], x_{i, 0}^{+}\right]+\left[\left[x_{j, s+r_{1}+r_{2}}^{+}, x_{i, 0}^{+}\right], x_{i, 1}^{+}\right]=2\left[\left[x_{j, s+r_{1}+r_{2}}^{+}, x_{i, 1}^{+}\right], x_{i, 0}^{+}\right] .
$$

Proposition 7.16. Relations $\left(x_{d}\right),\left(x_{1,2}\right)$ and $(s 2)$ are equivalent to relations $\left(x_{d}\right),\left(x_{1,2}\right)$ and $\left(s_{2}\right)$, where

$\left(s_{2}\right) \quad\left[\left[x_{j, s}^{+}, x_{i, 0}^{+}\right], x_{i, 1}^{+}\right]+\left[\left[x_{j, s+1}^{+}, x_{i, 0}^{+}\right], x_{i, 0}^{+}\right]=0 \quad\left(a_{i j}=-2, X_{\tilde{n}}^{(k)}=A_{2 n}^{(2)}\right)$. 
Proof. Indeed, Lemma 7.14(ii) implies that

$$
\begin{aligned}
\sum_{\sigma \in \mathcal{S}_{2}} \sigma \cdot\left(\left[\left[x_{j, s}^{+}, x_{i, r_{1}+1}^{+}\right], x_{i, r_{2}}^{+}\right]+\left[\left[x_{j, s}^{+}, x_{i, r_{2}}^{+}\right], x_{i, r_{1}+1}^{+}\right]\right) \\
=2\left(\left[\left[x_{j, s+r_{1}+r_{2}}^{+}, x_{i, 0}^{+}\right], x_{i, 1}^{+}\right]+\left[\left[x_{j, s+r_{1}+r_{2}+1}^{+}, x_{i, 0}^{+}\right], x_{i, 0}^{+}\right]\right) .
\end{aligned}
$$

Lemma 7.17. Relations $\left(x_{d}\right),\left(x_{1,2}\right)$ and $(t 3)$ are equivalent to relations $\left(x_{d}\right)$, $\left(x_{1,2}\right)$ and $(\tilde{t} 3)$, where

$(\tilde{t} 3) \quad\left[\left[x_{j, s}^{+}, x_{i, r_{1}+2}^{+}\right], x_{i, r_{2}}^{+}\right]+\left[\left[x_{j, s}^{+}, x_{i, r_{1}+1}^{+}\right], x_{i, r_{2}+1}^{+}\right]+\left[\left[x_{j, s}^{+}, x_{i, r_{1}}^{+}\right], x_{i, r_{2}+2}^{+}\right]=0$

$\left(k=3, a_{i j}=-3, s, r_{1}, r_{2}, r_{3} \in \mathbb{N}\right)$.

Proof. Indeed, by Corollary 7.11,

$$
\begin{aligned}
\sum_{\sigma \in \mathcal{S}_{2}} \sigma \cdot\left(2\left[\left[x_{j, s}^{+}, x_{i, r_{1}+2}^{+}\right], x_{i, r_{2}}^{+}\right]+\left[\left[x_{j, s}^{+}, x_{i, r_{1}+1}^{+}\right], x_{i, r_{2}+1}^{+}\right]\right) \\
=2\left(\left[\left[x_{j, s}^{+}, x_{i, r_{1}+2}^{+}\right], x_{i, r_{2}}^{+}\right]+\left[\left[x_{j, s}^{+}, x_{i, r_{1}}^{+}\right], x_{i, r_{2}+2}^{+}\right]+\left[\left[x_{j, s}^{+}, x_{i, r_{1}+1}^{+}\right], x_{i, r_{2}+1}^{+}\right]\right) .
\end{aligned}
$$

Notation 7.18. Let us define the following relations:

$$
\begin{array}{lcl}
\left(t_{3}^{\prime}\right) & {\left[\left[x_{j, s}^{+}, x_{i, 1}^{+}\right], x_{i, 1}^{+}\right]=-2\left[\left[x_{j, s}^{+}, x_{i, 2}^{+}\right], x_{i, 0}^{+}\right]} & \left(k=3, a_{i j}=-3\right), \\
\left(t_{3}^{\prime \prime}\right) & 2\left[\left[x_{j, s}^{+}, x_{i, 2}^{+}\right], x_{i, 1}^{+}\right]=-\left[\left[x_{j, s+3}^{+}, x_{i, 0}^{+}\right], x_{i, 0}^{+}\right] & \left(k=3, a_{i j}=-3\right), \\
\left(t_{3}^{\prime \prime \prime}\right) & {\left[\left[x_{j, s}^{+}, x_{i, 2}^{+}\right], x_{i, 2}^{+}\right]=-2\left[\left[x_{j, s+3}^{+}, x_{i, 1}^{+}\right], x_{i, 0}^{+}\right]} & \left(k=3, a_{i j}=-3\right) .
\end{array}
$$

Remark 7.19. Relations $\left(x_{d}\right),\left(x_{1,2}\right),(\tilde{t} 3)$ imply relations $\left(t_{3}^{\prime}\right)-\left(t_{3}^{\prime \prime \prime}\right)$.

Proof. Using relations $\left(x_{d}\right)$ and $\left(x_{1,2}\right)$ we find of course that $\left(t_{3}^{\prime}\right),\left(t_{3}^{\prime \prime}\right)$ and $\left(t_{3}^{\prime \prime \prime}\right)$ are $(\tilde{t} 3)$ with $r_{1}+r_{2}=0,1,2$ respectively.

Proposition 7.20. Relations $\left(x_{d}\right),\left(x_{1,2}\right),\left(t_{3}^{\prime}\right),\left(t_{3}^{\prime \prime}\right),\left(t_{3}^{\prime \prime \prime}\right)$ are equivalent to relations $\left(x_{d}\right),\left(x_{1,2}\right),(t 3)$.

Proof. We prove by induction on $r_{1}+r_{2}$ that relations $\left(x_{d}\right),\left(x_{1,2}\right),\left(t_{3}^{\prime}\right),\left(t_{3}^{\prime \prime}\right),\left(t_{3}^{\prime \prime \prime}\right)$ imply relations $(\tilde{t} 3)$, the cases $0 \leq r_{1}+r_{2}<3$ being obvious (see the proof of Remark 7.19). If $r_{1}+r_{2} \geq 3$ use induction on $r_{2}$ : if $r_{2}=0$ then $r_{1} \geq 3$ and thanks to $\left(x_{d}\right)$ we have

$$
\begin{aligned}
& {\left[\left[x_{j, s}^{+}, x_{i, r_{1}+2}^{+}\right], x_{i, r_{2}}^{+}\right]+\left[\left[x_{j, s}^{+}, x_{i, r_{1}+1}^{+}\right], x_{i, r_{2}+1}^{+}\right]+\left[\left[x_{j, s}^{+}, x_{i, r_{1}}^{+}\right], x_{i, r_{2}+2}^{+}\right]} \\
& =\left[\left[x_{j, s+3}^{+}, x_{i, r_{1}-1}^{+}\right], x_{i, 0}^{+}\right]+\left[\left[x_{j, s+3}^{+}, x_{i, r_{1}-2}^{+}\right], x_{i, 1}^{+}\right]+\left[\left[x_{j, s+3}^{+}, x_{i, r_{1}-3}^{+}\right], x_{i, 2}^{+}\right]
\end{aligned}
$$

which is zero by the inductive hypothesis $\left(r_{1}-3+0<r_{1}+r_{2}\right)$; if $r_{2}>0$ then, thanks to Lemma 7.14(iii), $\left[\left[x_{j, s}^{+}, x_{i, r_{1}}^{+}\right], x_{i, r_{2}+2}^{+}\right]=\left[\left[x_{j, s}^{+}, x_{i, r_{1}+3}^{+}\right], x_{i, r_{2}-1}^{+}\right]$, so that 


$$
\begin{aligned}
& {\left[\left[x_{j, s}^{+}, x_{i, r_{1}+2}^{+}\right], x_{i, r_{2}}^{+}\right]+\left[\left[x_{j, s}^{+}, x_{i, r_{1}+1}^{+}\right], x_{i, r_{2}+1}^{+}\right]+\left[\left[x_{j, s}^{+}, x_{i, r_{1}}^{+}\right], x_{i, r_{2}+2}^{+}\right]} \\
& =\left[\left[x_{j, s}^{+}, x_{i, r_{1}+3}^{+}\right], x_{i, r_{2}-1}^{+}\right]+\left[\left[x_{j, s}^{+}, x_{i, r_{1}+2}^{+}\right], x_{i, r_{2}}^{+}\right]+\left[\left[x_{j, s}^{+}, x_{i, r_{1}+1}^{+}\right], x_{i, r_{2}+1}^{+}\right],
\end{aligned}
$$

which is zero because $r_{2}-1<r_{2}$.

Remark 7.21. If $k=3, a_{i j}=-3$, relations $\left(x_{d}\right),\left(x_{1,2}\right),\left(t_{3}^{\prime}\right),\left(t_{3}^{\prime \prime}\right),\left(t_{3}^{\prime \prime \prime}\right)$ imply that if $s>0$ then

$$
\left[\left[\left[x_{j, s}^{+}, x_{i, 1}^{+}\right], x_{i, 0}^{+}\right], x_{i, 0}^{+}\right]=0, \quad\left[\left[\left[x_{j, s}^{+}, x_{i, 2}^{+}\right], x_{i, 0}^{+}\right], x_{i, 0}^{+}\right]=0 .
$$

Proof. If $s>0$ then $s \geq 3$, so relations $\left(t_{3}^{\prime \prime \prime}\right),\left(t_{3}^{\prime}\right),\left(t_{3}^{\prime \prime}\right)$ imply that

$$
\begin{array}{r}
2\left[\left[\left[x_{j, s}^{+}, x_{i, 1}^{+}\right], x_{i, 0}^{+}\right], x_{i, 0}^{+}\right]+\left[\left[\left[x_{j, s-3}^{+}, x_{i, 2}^{+}\right], x_{i, 2}^{+}\right], x_{i, 0}^{+}\right]=0, \\
2\left[\left[\left[x_{j, s-3}^{+}, x_{i, 2}^{+}\right], x_{i, 0}^{+}\right], x_{i, 2}^{+}\right]+\left[\left[\left[x_{j, s-3}^{+}, x_{i, 1}^{+}\right], x_{i, 1}^{+}\right], x_{i, 2}^{+}\right]=0, \\
2\left[\left[\left[x_{j, s-3}^{+}, x_{i, 2}^{+}\right], x_{i, 1}^{+}\right], x_{i, 1}^{+}\right]+\left[\left[\left[x_{j, s}^{+}, x_{i, 0}^{+}\right], x_{i, 0}^{+}\right], x_{i, 1}^{+}\right]=0,
\end{array}
$$

from which, thanks to Corollary 7.11,

$$
9\left[\left[\left[x_{j, s}^{+}, x_{i, 1}^{+}\right], x_{i, 0}^{+}\right], x_{i, 0}^{+}\right]=0 .
$$

Analogously

$$
\begin{aligned}
2\left[\left[\left[x_{j, s}^{+}, x_{i, 2}^{+}\right], x_{i, 0}^{+}\right], x_{i, 0}^{+}\right]+\left[\left[\left[x_{j, s}^{+}, x_{i, 1}^{+}\right], x_{i, 1}^{+}\right], x_{i, 0}^{+}\right] & =0, \\
2\left[\left[\left[x_{j, s}^{+}, x_{i, 1}^{+}\right], x_{i, 0}^{+}\right], x_{i, 1}^{+}\right]+\left[\left[\left[x_{j, s-3}^{+}, x_{i, 2}^{+}\right], x_{i, 2}^{+}\right], x_{i, 1}^{+}\right] & =0, \\
2\left[\left[\left[x_{j, s-3}^{+}, x_{i, 2}^{+}\right], x_{i, 1}^{+}\right], x_{i, 2}^{+}\right]+\left[\left[\left[x_{j, s}^{+}, x_{i, 0}^{+}\right], x_{i, 0}^{+}\right], x_{i, 2}^{+}\right] & =0,
\end{aligned}
$$

which yields $\left[\left[\left[x_{j, s}^{+}, x_{i, 2}^{+}\right], x_{i, 0}^{+}\right], x_{i, 0}^{+}\right]=0$.

Notation 7.22. Let us define the following relations:

$$
\begin{array}{ll}
{\left[\left[\left[x_{j, 0}^{+}, x_{i, 1}^{+}\right], x_{i, 0}^{+}\right], x_{i, 0}^{+}\right]=0} & \left(k=3, a_{i j}=-3\right), \\
{\left[\left[\left[x_{j, 0}^{+}, x_{i, 2}^{+}\right], x_{i, 0}^{+}\right], x_{i, 0}^{+}\right]=0} & \left(k=3, a_{i j}=-3\right) .
\end{array}
$$

Remark 7.23. Relations $\left(x_{1,2}\right),\left(t_{3}^{\prime}\right)$ and $(u 3)$ imply relations $\left(u_{3}^{\prime}\right)$ and $\left(u_{3}^{\prime \prime}\right)$.

Proof. $\left(u_{3}^{\prime}\right)$ is (u3) with $s=r_{1}=r_{2}=r_{3}=0$.

$\left[\left(t_{3}^{\prime}\right), x_{i, 0}^{+}\right]$with $s=0$ and $(u 3)$ with $\left(s, r_{1}, r_{2}, r_{3}\right)=(0,1,0,0)$ imply $\left(u_{3}^{\prime \prime}\right)$, using Corollary 7.11.

Proposition 7.24. Relations $\left(x_{d}\right),\left(x_{1,2}\right),\left(t_{3}^{\prime}\right),\left(t_{3}^{\prime \prime}\right),\left(t_{3}^{\prime \prime \prime}\right),\left(u_{3}^{\prime}\right),\left(u_{3}^{\prime \prime}\right)$ imply relations (u3) (hence are equivalent to relations $\left.\left(x_{d}\right),\left(x_{1,2}\right),(t 3),(u 3)\right)$.

Proof. The hypotheses imply that $\left[\left[\left[x_{j, s}^{+}, x_{i, r_{1}}^{+}\right], x_{i, r_{2}}^{+}\right], x_{i, r_{3}}^{+}\right]$is a rational multiple of $\left[\left[\left[x_{j, s}^{+}, x_{i, r_{1}+r_{2}+r_{3}}^{+}\right], x_{i, 0}^{+}\right], x_{i, 0}^{+}\right]$(by $\left.\left(x_{d}\right),\left(x_{1,2}\right),\left(t_{3}^{\prime}\right),\left(t_{3}^{\prime \prime}\right),\left(t_{3}^{\prime \prime \prime}\right)\right)$, which is zero if 
$3 \nmid r_{1}+r_{2}+r_{3}$ (by $\left(x_{d}\right),\left(u_{3}^{\prime}\right),\left(u_{3}^{\prime \prime}\right)$ and Lemma 7.21 ). In particular (u3) holds if $3 \nmid r_{1}+r_{2}+r_{3}+1$. Otherwise we can suppose $0 \leq r_{1}, r_{2}, r_{3}<3, r_{1}=r_{2}$, $r_{3}+1 \equiv r_{1}(\bmod 3)\left(\right.$ thanks to $\left(x_{d}\right)$ and $\left.\left(x_{1,2}\right)\right)$, or equivalently that $\left(r_{1}, r_{2}, r_{3}\right)=$ $(1,1,0),(2,0,0),(2,2,1)$. In these cases $(u 3)$ corresponds to $\left[\left(t_{3}^{\prime}\right), x_{i, 1}^{+}\right],\left[\left(t_{3}^{\prime \prime}\right), x_{i, 0}^{+}\right]$, and $\left[\left(t_{3}^{\prime \prime \prime}\right), x_{i, 2}^{+}\right]$respectively.

Notation 7.25. Let us define the following relations:

(serre)

$$
\left(\operatorname{ad} x_{i, 0}^{+}\right)^{1-a_{i j}}\left(x_{j, s}^{+}\right)=0 \quad(i \neq j) .
$$

Remark 7.26. Relation $(s)$ implies relation (serre).

Proof. The claim is obvious since (serre) is $(s)$ with $r_{u}=0$ for all $u=1, \ldots, 1-a_{i j}$.

Proposition 7.27. Relations $\left(x_{d}\right),\left(x_{1,2}\right),\left(x_{3}\right),\left(t_{2}^{\prime}\right),\left(t_{2}^{\prime \prime}\right),\left(s_{2}\right),\left(t_{3}^{\prime}\right),\left(t_{3}^{\prime \prime}\right),\left(t_{3}^{\prime \prime \prime}\right)$, $\left(u_{3}^{\prime}\right),\left(u_{3}^{\prime \prime}\right),($ serre $)$ imply that $\left[\left[\ldots\left[x_{j, s}^{+}, x_{i, r_{1}}^{+}\right], \ldots\right], x_{i, r_{1-a_{i j}}}^{+}\right]=0$ for all $s \in \mathbb{Z}$ and $r \in \mathbb{Z}^{1-a_{i j}}$; in particular they imply relation $(s)$.

Proof. The relations, Corollary 7.11 and Lemma 7.14 imply that

$$
\left[\left[\ldots\left[x_{j, s}^{+}, x_{i, r_{1}}^{+}\right], \ldots\right], x_{i, r_{1-a_{i j}}}^{+}\right]
$$

is a rational multiple of

$$
\begin{aligned}
& {\left[\left[\ldots\left[\left[x_{j, s}^{+}, x_{i, r_{1}+\cdots+r_{1-a_{i j}}}^{+}\right], x_{i, 0}^{+}\right], \ldots\right], x_{i, 0}^{+}\right]} \\
& \quad= \begin{cases}\left(-\operatorname{ad} x_{i, 0}^{+}\right)^{1-a_{i j}}\left(x_{j, s+r_{1}+\cdots+r_{1-a_{i j}}^{+}}^{+}\right) & \text {if } \tilde{d}_{j} \mid r_{1}+\cdots+r_{1-a_{i j}}, \\
0 & \text { otherwise }\end{cases}
\end{aligned}
$$

(hence zero by (serre)) apart from the case $A_{2 n}^{(2)}, a_{i j}=-2, r_{3}$ odd, when

$$
\left[\left[\left[x_{j, s}^{+}, x_{i, r_{1}}^{+}\right], x_{i, r_{2}}^{+}\right], x_{i, r_{3}}^{+}\right]=(-1)^{r_{2}}\left[\left[\left[x_{j, s+r_{1}+r_{2}}^{+}, x_{i, 0}^{+}\right], x_{i, 0}^{+}\right], x_{i, r_{3}}^{+}\right] .
$$

But by the above considerations

$$
\begin{aligned}
{\left[\left[\left[x_{j, s}^{+}, x_{i, 0}^{+}\right], x_{i, 0}^{+}\right], x_{i, r}^{+}\right] } & =\left[\left[x_{j, s}^{+}, x_{i, 0}^{+}\right],\left[x_{i, 0}^{+}, x_{i, r}^{+}\right]\right]+\left[\left[\left[x_{j, s}^{+}, x_{i, 0}^{+}\right], x_{i, r}^{+}\right], x_{i, 0}^{+}\right] \\
& =\left[x_{j, s}^{+},\left[x_{i, 0}^{+},\left[x_{i, 0}^{+}, x_{i, r}^{+}\right]\right]\right]+\left[\left[x_{j, s}^{+},\left[x_{i, 0}^{+}, x_{i, r}^{+}\right]\right], x_{i, 0}^{+}\right] \\
& =\left[\left[\left[x_{j, s}^{+}, x_{i, 0}^{+}\right], x_{i, r}^{+}\right], x_{i, 0}^{+}\right]-\left[\left[\left[x_{j, s}^{+}, x_{i, r}^{+}\right], x_{i, 0}^{+}\right], x_{i, 0}^{+}\right]=0 .
\end{aligned}
$$

Thus $\left[\left[\ldots\left[x_{j, s}^{+}, x_{i, r_{1}}^{+}\right], \ldots\right], x_{i, r_{1-a_{i j}}}^{+}\right]=0$ always.

Remark 7.28. It is worth remarking that in the cases $k>1, a_{i j}=-k$ relations $(z x),\left(x_{d}\right),\left(x_{1,2}\right),\left(x_{3}\right),\left(t_{2}^{\prime}\right),\left(t_{2}^{\prime \prime}\right),\left(s_{2}\right),\left(t_{3}^{\prime}\right),\left(t_{3}^{\prime \prime}\right),\left(t_{3}^{\prime \prime \prime}\right)$ imply (serre) with $s \geq k$ (that is, $s>0$ if $X_{\tilde{n}}^{(k)} \neq A_{2 n}^{(2)}$ and $s>1$ if $X_{\tilde{n}}^{(k)}=A_{2 n}^{(2)}$ ). Compare this observation with Remark $3.10($ iii)\&(iv). 
Proof. Suppose $k=2, X_{\tilde{n}}^{(k)} \neq A_{2 n}^{(2)}$. If $s>0$ then $s \geq 2$, hence

$$
\begin{aligned}
{\left[\left[\left[x_{j, s}^{+}, x_{i, 0}^{+}\right], x_{i, 0}^{+}\right], x_{i, 0}^{+}\right] } & =-\left[\left[\left[x_{j, s-2}^{+}, x_{i, 1}^{+}\right], x_{i, 1}^{+}\right], x_{i, 0}^{+}\right] \\
& =-\left[\left[\left[x_{j, s-2}^{+}, x_{i, 1}^{+}\right], x_{i, 0}^{+}\right], x_{i, 1}^{+}\right]=0 .
\end{aligned}
$$

If $X_{\tilde{n}}^{(k)}=A_{2 n}^{(2)}$ let $r<s$; then

$$
\begin{aligned}
{\left[\left[\left[x_{j, s}^{+}, x_{i, 0}^{+}\right], x_{i, 0}^{+}\right], x_{i, 0}^{+}\right]=} & -\left[\left[\left[x_{j, s-r-1}^{+}, x_{i, r}^{+}\right], x_{i, 1}^{+}\right], x_{i, 0}^{+}\right] \\
= & -\left[\left[\left[x_{j, s-r-1}^{+}, x_{i, r}^{+}\right], x_{i, 0}^{+}\right], x_{i, 1}^{+}\right]-\left[\left[x_{j, s-r-1}^{+}, x_{i, r}^{+}\right],\left[x_{i, 1}^{+}, x_{i, 0}^{+}\right]\right] \\
= & -\left[\left[\left[x_{j, s-1}^{+}, x_{i, 0}^{+}\right], x_{i, 0}^{+}\right], x_{i, 1}^{+}\right]-\left[\left[x_{j, s-r-1}^{+},\left[x_{i, 1}^{+}, x_{i, 0}^{+}\right]\right], x_{i, r}^{+}\right] \\
= & -\left[\left[\left[x_{j, s-1}^{+}, x_{i, 0}^{+}\right], x_{i, 0}^{+}\right], x_{i, 1}^{+}\right]-\left[\left[\left[x_{j, s-r-1}^{+}, x_{i, 1}^{+}\right], x_{i, 0}^{+}\right], x_{i, r}^{+}\right] \\
& +\left[\left[\left[x_{j, s-r-1}^{+}, x_{i, 0}^{+}\right], x_{i, 1}^{+}\right], x_{i, r}^{+}\right] \\
= & -\left[\left[\left[x_{j, s-1}^{+}, x_{i, 0}^{+}\right], x_{i, 0}^{+}\right], x_{i, 1}^{+}\right]-2\left[\left[\left[x_{j, s-r}^{+}, x_{i, 0}^{+}\right], x_{i, 0}^{+}\right], x_{i, r}^{+}\right] ;
\end{aligned}
$$

in particular if $s \geq 2$ we have (choosing $r=0,1$ )

$$
\begin{aligned}
& 3\left[\left[\left[x_{j, s}^{+}, x_{i, 0}^{+}\right], x_{i, 0}^{+}\right], x_{i, 0}^{+}\right]+\left[\left[\left[x_{j, s-1}^{+}, x_{i, 0}^{+}\right], x_{i, 0}^{+}\right], x_{i, 1}^{+}\right]=0, \\
& {\left[\left[\left[x_{j, s}^{+}, x_{i, 0}^{+}\right], x_{i, 0}^{+}\right], x_{i, 0}^{+}\right]+3\left[\left[\left[x_{j, s-1}^{+}, x_{i, 0}^{+}\right], x_{i, 0}^{+}\right], x_{i, 1}^{+}\right]=0,}
\end{aligned}
$$

from which the claim follows.

Now suppose $k=3$. Then $s>0$ implies $s \geq 3$, hence

$$
\begin{aligned}
{\left[\left[\left[\left[x_{j, s}^{+}, x_{i, 0}^{+}\right], x_{i, 0}^{+}\right], x_{i, 0}^{+}\right], x_{i, 0}^{+}\right] } & =-2\left[\left[\left[\left[x_{j, s-3}^{+}, x_{i, 2}^{+}\right], x_{i, 1}^{+}\right], x_{i, 0}^{+}\right], x_{i, 0}^{+}\right] \\
& =-2\left[\left[\left[\left[x_{j, s-3}^{+}, x_{i, 2}^{+}\right], x_{i, 0}^{+}\right], x_{i, 0}^{+}\right], x_{i, 1}^{+}\right]=0 .
\end{aligned}
$$

Corollary 7.29. $L_{+}$is the Lie algebra generated by $\left\{x_{i, r}^{+} \mid i \in I_{0}, r \geq 0\right\}$ with relations $(z x),\left(x_{d}\right),\left(x_{1,2}\right),\left(x_{3}\right),\left(t_{2}^{\prime}\right),\left(t_{2}^{\prime \prime}\right),\left(s_{2}\right),\left(t_{3}^{\prime}\right),\left(t_{3}^{\prime \prime}\right),\left(t_{3}^{\prime \prime \prime}\right),\left(u_{3}^{\prime}\right),\left(u_{3}^{\prime \prime}\right),($ serre $)$.

\section{§8. Affine Kac-Moody case}

This section is devoted to the study of the Lie algebra $L_{+}$(see Definition 7.4) and of its relation, through $\tilde{\psi}_{1}$ (see Remarks 7.5 and 7.6 and Proposition 7.7), with the Kac-Moody algebra $\hat{\mathfrak{g}}$ (see Corollary 8.21).

Proposition 7.7 and the structure of the root system of $\hat{\mathfrak{g}}$ (see Remark 1.2) imply that in order to prove that $\left.\tilde{\psi}_{1}\right|_{L_{+}}$is injective it is enough to show that for all $\alpha \in Q_{0,+} \backslash\{0\}$ and $r \in \mathbb{N}$,

$$
\operatorname{dim}_{\mathbb{C}}\left(L_{+}\right)_{\alpha+r \delta} \leq \operatorname{dim}_{\mathbb{C}} \hat{\mathfrak{g}}_{\alpha+r \delta}= \begin{cases}1 & \text { if } \alpha+r \delta \in \Phi_{+}^{\mathrm{re}} \\ 0 & \text { otherwise }\end{cases}
$$

Notice that the results of $\S 7$ imply the following: 
Proposition 8.1. If $i \in I_{0}$ and $r \in \mathbb{N}$ then:

$$
\begin{aligned}
\operatorname{dim}\left(L_{+}\right)_{\alpha_{i}+r \delta} \leq \begin{cases}1 & \text { if } \tilde{d}_{i} \mid r, \\
0 & \text { otherwise, }\end{cases} \\
\operatorname{dim}\left(L_{+}\right)_{2 \alpha_{i}+r \delta} \leq \begin{cases}1 & \text { if }\left(X_{\tilde{n}}^{(k)}, d_{i}\right)=\left(A_{2 n}^{(2)}, 1\right) \text { and } 2 \nmid r, \\
0 & \text { otherwise, }\end{cases} \\
\operatorname{dim}\left(L_{+}\right)_{h \alpha_{i}+r \delta}=0 \quad \text { if } h>2 .
\end{aligned}
$$

Proof. $\left(L_{+}\right)_{\alpha_{i}+r \delta}=\left\langle x_{i, r}^{+}\right\rangle$for all $r \in \mathbb{N}$ and, for all $h>1$ and $r \in \mathbb{N}$,

$$
\left(L_{+}\right)_{h \alpha_{i}+r \delta}=\left\langle\left[\left(L_{+}\right)_{(h-1) \alpha_{i}+r_{1} \delta}, x_{i, r_{2}}^{+}\right] \mid r_{1}+r_{2}=r\right\rangle ;
$$

in particular:

(i) $\left(L_{+}\right)_{h \alpha_{i}+r \delta}=\{0\}$ for all $r \in \mathbb{N}$ implies $L_{\tilde{h} \alpha_{i}+r \delta}=\{0\}$ for all $r \in \mathbb{N}$ and $\tilde{h} \geq h$;

(ii) $\left(L_{+}\right)_{h \alpha_{i}+r \delta}=\{0\}$ for all $h>0, i \in I_{0}$ and $r \in \mathbb{N}$ such that $\tilde{d}_{i} \nmid r$; in particular (D1) holds;

(iii) (D2) follows from Lemma 7.10;

(iv) (D3) follows from Lemma 7.12.

In order to generalize this result to all the roots we embed $L_{+}$into a $\mathfrak{g}_{0^{-}}$ module $L$; this structure provides the symmetries that allow one to easily determine the needed dimensions of the homogeneous components of $L_{+}$.

Definition 8.2. (i) $L_{0}$ is the abelian Lie algebra generated by $\left\{h_{i, r} \mid i \in I_{0}\right.$, $r \in \mathbb{N}\}$ with relations $h_{i, r}=0$ if $\tilde{d}_{i} \nmid r$ (hence $\left\{h_{i, r}\left|i \in I_{0}, \tilde{d}_{i}\right| r \in \mathbb{N}\right\}$ is a basis of $\left.L_{0}\right)$;

(ii) $L_{-}=\left(L_{+}\right)^{\mathrm{op}}$;

(iii) for all $i \in I_{0}$ and $r \in \mathbb{N}, x_{i, r}^{-}$denotes $-x_{i, r}^{+}$as an element of $L_{-}$;

(iv) $L=L_{-} \oplus L_{0} \oplus L_{+}$;

(v) $\sigma: L \rightarrow L$ is the linear map defined by $L=L_{-} \oplus L_{0} \oplus L_{+} \ni(y, h, x) \mapsto$ $(x, h, y) \in L_{-} \oplus L_{0} \oplus L_{+}=L$ (in particular $\left.\sigma\left(h_{i, r}\right)=h_{i, r}, \sigma\left(x_{i, r}^{ \pm}\right)=-x_{i, r}^{\mp}\right)$.

Remark 8.3. (i) $L_{0}=L_{0}^{\mathrm{op}}$ as Lie algebras (since $L_{0}$ is abelian);

(ii) $\left.\sigma\right|_{L_{+}}: L_{+} \rightarrow L_{-},\left.\sigma\right|_{L_{-}}: L_{-} \rightarrow L_{+}$and $\left.\sigma\right|_{L_{0}}=\mathrm{id}_{L_{0}}: L_{0} \rightarrow L_{0}$ are antiisomorphisms of Lie algebras.

Remark 8.4. (i) $\mathfrak{h}_{0} \ni h_{i} \mapsto h_{i, 0} \in L_{0}$ defines a homomorphism of Lie algebras;

(ii) $\mathfrak{g}_{0,+} \ni e_{i} \mapsto x_{i, 0}^{+} \in L_{+}$defines a homomorphism of Lie algebras, hence it induces an action of $\mathfrak{g}_{0,+}$ on $L_{+}\left(e_{i} \mapsto \operatorname{ad}_{L_{+}} x_{i, 0}^{+}\right)$, and $\operatorname{ad}_{L_{+}} x_{i, 0}^{+}$is locally nilpotent; 
(iii) $\mathfrak{g}_{0,-} \ni f_{i} \mapsto x_{i, 0}^{-} \in L_{-}$defines a homomorphism of Lie algebras, hence it induces an action of $\mathfrak{g}_{0,-}$ on $L_{-}\left(f_{i} \mapsto \operatorname{ad}_{L_{-}} x_{i, 0}^{-}\right)$, and $\operatorname{ad}_{L_{-}} x_{i, 0}^{-}$is locally nilpotent.

Proposition 8.5.

$h_{i, r} \cdot x_{j, s}^{+}=B_{i j r} x_{j, r+s}^{+}$with $B_{i j r}= \begin{cases}0 & \text { if } \tilde{d}_{i, j} \nmid r, \\ 2\left(2-(-1)^{r}\right) & \text { if }\left(X_{\tilde{n}}^{(k)}, d_{i}, d_{j}\right)=\left(A_{2 n}^{(2)}, 1,1\right), \\ a_{i j} & \text { otherwise, }\end{cases}$

defines a Lie algebra homomorphism $D_{+}: L_{0} \rightarrow \operatorname{Der}\left(L_{+}\right)$. Then $L_{0} \oplus L_{+}=$ $L_{0} \ltimes_{D_{+}} L_{+}$is endowed with a Lie algebra structure (semidirect product of $L_{0}$ and $\left.L_{+}\right)$. Of course $\left(L_{0} \ltimes_{D_{+}} L_{+}\right)^{\mathrm{op}}=L_{-} \oplus L_{0}$ is a Lie algebra.

Proof. It is obvious that for all $i \in I_{0}$ and $r \in \mathbb{N}$ the ideal generated by the relations $\left(\tilde{d} r_{+}\right)$is stable under the derivation $x_{j, s}^{+} \mapsto B_{i j r} x_{j, r+s}^{+}$(see also [Da1]), hence $h_{i, r}$. defines a derivation of $L_{+}$; it is also immediate to see that $h_{i, r} .=0$ if $\tilde{d}_{i} \nmid r$ and that $h_{i, r} . h_{j, s} .=h_{j, s} . h_{i, r}$, hence the map $h_{i, r} \mapsto h_{i, r}$. induces a Lie algebra homomorphism $D_{+}: L_{0} \rightarrow \operatorname{Der}\left(L_{+}\right)$.

Remark 8.6. (i) $\left.\sigma\right|_{L_{0} \oplus L_{+}}: L_{0} \oplus L_{+} \rightarrow L_{-} \oplus L_{0}$ and $\left.\sigma\right|_{L_{-} \oplus L_{0}}: L_{-} \oplus L_{0} \rightarrow$ $L_{0} \oplus L_{+}$are anti-isomorphisms of Lie algebras;

(ii) $\left.\sigma \circ\left(\operatorname{ad}_{L_{0} \oplus L_{+}} a\right) \circ \sigma\right|_{L_{-} \oplus L_{0}}=-\left.\left(\operatorname{ad}_{L_{-} \oplus L_{0}} \sigma(a)\right)\right|_{L_{-} \oplus L_{0}}$ for all $a \in L_{0} \oplus L_{+}$;

(iii) $\mathfrak{h}_{0} \oplus \mathfrak{g}_{0,+} \rightarrow L_{0} \oplus L_{+}$and $\mathfrak{g}_{0,-} \oplus \mathfrak{h}_{0} \rightarrow L_{-} \oplus L_{0}$ are homomorphisms of Lie algebras (indeed $B_{i j 0}=a_{i j}$ ); in particular they induce actions of $\mathfrak{h}_{0} \oplus \mathfrak{g}_{0,+}$ on $L_{0} \oplus L_{+}\left(h_{i} \mapsto \operatorname{ad}_{L_{0} \oplus L_{+}} h_{i, 0}, e_{i} \mapsto \operatorname{ad}_{L_{0} \oplus L_{+}} x_{i, 0}^{+}\right)$and of $\mathfrak{g}_{0,-} \oplus \mathfrak{h}_{0}$ on $L_{-} \oplus L_{0}$ $\left(f_{i} \mapsto \operatorname{ad}_{L_{-} \oplus L_{0}} x_{i, 0}^{-}, h_{i} \mapsto \operatorname{ad}_{L_{-} \oplus L_{0}} h_{i, 0}\right) ;$

(iv) for all $i \in I_{0},\left.\operatorname{ad}_{L_{0} \oplus L_{+}} x_{i, 0}^{+}\right|_{L_{0}}$ maps $L_{0}$ in $L_{+}\left(L_{+}\right.$is an ideal of $\left.L_{0} \oplus L_{+}\right)$, hence $\operatorname{ad}_{L_{0} \oplus L_{+}} x_{i, 0}^{+}$is locally nilpotent, since it is locally nilpotent on $L_{+}$(see Remark 8.4(ii)); analogously $\operatorname{ad}_{L_{-} \oplus L_{0}} x_{i, 0}^{-}$is locally nilpotent;

(v) for all $h \in L_{0}$,

$\left.\operatorname{ad}_{L_{0} \oplus L_{+}} h\right|_{L_{0}}=0=\left.\operatorname{ad}_{L_{-} \oplus L_{0}} h\right|_{L_{0}},\left.\sigma \circ \operatorname{ad}_{L_{0} \oplus L_{+}} h \circ \sigma\right|_{L_{-}}=-\left.\operatorname{ad}_{L_{-} \oplus L_{0}} h\right|_{L_{-}} ;$

in particular the adjoint actions of $L_{0}$ on $L_{0} \oplus L_{+}$and on $L_{-} \oplus L_{0}$ coincide on $L_{0}$ and thus define an $L_{0}$-module structure on $L$ (denoted by $h \mapsto h_{L}$ ) such that $\sigma \circ h_{L} \circ \sigma=-h_{L}$;

(vi) $\mathfrak{h}_{0}\left(\subseteq L_{0}\right)$ acts diagonally on $L$ and trivially on $L_{0}$; more precisely $L_{ \pm}$and $L_{0}$, hence $L$, are $Q$-graded $\left(x_{i, r}^{ \pm} \in\left(L_{ \pm}\right)_{ \pm \alpha_{i}+r \delta}=L_{ \pm \alpha_{i}+r \delta}\right.$ and $h_{i, r} \in\left(L_{0}\right)_{r \delta}=$ $\left.L_{r \delta}\right)$ and $h \in \mathfrak{h}_{0}$ acts on $L_{\alpha}$ as $\alpha(h) \operatorname{id}_{L_{\alpha}}$;

(vii) the action of $\mathfrak{h}_{0} \oplus \mathfrak{g}_{0,+}$ on $L_{0} \oplus L_{+}$and that of $\mathfrak{g}_{0,-} \oplus \mathfrak{h}_{0}$ on $L_{-} \oplus L_{0}$ are obviously homogeneous. 
Remark 8.7. We want to provide $L$ with a $\mathfrak{g}_{0}$-module structure extending the $\mathfrak{h}_{0^{-}}$ module structure (Remark 8.6(vi)), compatible with the $\mathfrak{h}_{0} \oplus \mathfrak{g}_{0, \pm}$-module structure on $L_{0} \oplus L_{ \pm}$(Remark 8.6(iii)), and homogeneous with respect to the $Q$-grading.

Remark 8.8. Let $e_{i, L}, f_{i, L}: L \rightarrow L$ be homogeneous linear maps (that is, $e_{i, L}\left(L_{\alpha}\right) \subseteq L_{\alpha+\alpha_{i}}$ and $\left.f_{i, L}\left(L_{\alpha}\right) \subseteq L_{\alpha-\alpha_{i}}\right)$. Then:

(i) the relations $\left[\left(h_{i}\right)_{L}, e_{j, L}\right]=a_{i j} e_{j, L},\left[\left(h_{i}\right)_{L}, f_{j, L}\right]=-a_{i j} f_{j, L}$ are automatically satisfied (because of the diagonal action of $\mathfrak{h}_{0}$ on $L$, see Remark 8.6(vi));

(ii) if moreover $\left.e_{i, L}\right|_{L_{+}}=\operatorname{ad}_{L_{+}} x_{i, 0}^{+}$and $\left.f_{i, L}\right|_{L_{-}}=\operatorname{ad}_{L_{-}} x_{i, 0}^{-}$, then $e_{i, L}$ and $f_{i, L}$ are locally nilpotent (see Remark 8.4(ii)\&(iii) and notice that for all $x \in L$ there exists $m \in \mathbb{N}$ such that $e_{i, L}^{m}(x) \in L_{+}$and $\left.f_{i, L}^{m}(x) \in L_{-}\right)$;

(iii) for all $r \in \mathbb{N}, L^{(r)}=\bigoplus_{\alpha \in Q_{0}} L_{\alpha+r \delta}$ is $e_{i, L^{-}}$and $f_{i, L^{-}}$-stable, and $L=\bigoplus_{r \in \mathbb{N}} L^{(r)}$.

Definition 8.9. Given $i \in I_{0}$ let $f_{i, L_{+}}: L_{+} \rightarrow L_{0} \oplus L_{+}$be the derivation defined on generators by $f_{i, L_{+}}\left(x_{j, r}^{+}\right)=-\delta_{i, j} h_{i, r}$, and let $e_{i, L_{-}}: L_{-} \rightarrow L_{-} \oplus L_{0}$ be defined by $e_{i, L_{-}}=\left.\sigma \circ f_{i, L_{+}} \circ \sigma\right|_{L_{-}}$.

Proposition 8.10. $f_{i, L_{+}}$and $e_{i, L_{-}}$are well defined derivations.

Proof. Obviously if $\rho$ is a relation involving only indices in $I_{0} \backslash\{i\}$ then $f_{i, L_{+}}(\rho)$ $=0$; it is also obvious that if $\tilde{d}_{j} \nmid r f_{i, L_{+}}\left(x_{j, r}^{+}\right)=-\delta_{i j} h_{j r}=0$ (hence $f_{i, L_{+}}$preserves relation $(z x))$. Moreover:

(i) if $i \neq j f_{i, L_{+}}\left(\left[x_{i, r}^{+}, x_{j, s}^{+}\right]\right)=-a_{i j} x_{j, r+s}^{+}$, which depends only on $r+s$, hence relation $\left(x_{d}\right)$ is preserved by $f_{i, L_{+}}$and symmetrically by $f_{j, L_{+}}$;

(ii) $f_{i, L_{+}}\left((-1)^{s}\left[x_{i, r}^{+}, x_{i, s}^{+}\right]\right)=(-1)^{s}\left(-B_{i i r}+B_{i i s}\right) x_{i, r+s}^{+}$, which is zero if $\left(X_{\tilde{n}}^{(k)}, d_{i}\right)$ $\neq\left(A_{2 n}^{(2)}, 1\right)$ or $2 \mid r+s$ and in any case depends only on $r+s$, hence relation $\left(x_{1,2}\right)$ is preserved by $f_{i, L_{+}}$;

(iii) if $\left(X_{\tilde{n}}^{(k)}, d_{i}\right)=\left(A_{2 n}^{(2)}, 1\right)$ then

$$
f_{i, L_{+}}\left(\left[\left[x_{i, r_{1}}^{+}, x_{i, r_{2}}^{+}\right], x_{i, r_{3}}^{+}\right]\right)=\left[f_{i, L_{+}}\left(\left[x_{i, r_{1}}^{+}, x_{i, r_{2}}^{+}\right]\right), x_{i, r_{3}}^{+}\right]+\left[\left[x_{i, r_{1}}^{+}, x_{i, r_{2}}^{+}\right], f_{i, L_{+}}\left(x_{i, r_{3}}^{+}\right)\right],
$$

which, if $2 \mid r_{1}+r_{2}$ or $2 \mid r_{1}+r_{2}+r_{3}$, is of course zero by (ii) and relation $\left(x_{1,2}\right)$, while it is $(-6+2)\left[x_{i, r_{1}+r_{2}}^{+}, x_{i, r_{3}}^{+}\right]+2\left[x_{i, r_{1}+r_{3}}^{+}, x_{i, r_{2}}^{+}\right]+2\left[x_{i, r_{1}}^{+}, x_{i, r_{2}+r_{3}}^{+}\right]=0$ if $2 \nmid r_{1}, 2 \mid r_{2}$ and $2 \mid r_{3}$, by relation $\left(x_{1,2}\right)$. It follows that $f_{i, L_{+}}$also preserves relations $\left(x_{1,2}, x_{3}\right)$.

Furthermore if $k>1$ and $a_{i j}=-k$ :

(iv) $f_{j, L_{+}}\left((-1)^{r_{2}}\left[\left[x_{j, s}^{+}, x_{i, r_{1}}^{+}\right], x_{i, r_{2}}^{+}\right]\right)=-(-1)^{r_{2}} a_{j i}\left[x_{i, s+r_{1}}^{+}, x_{i, r_{2}}^{+}\right]$, which is zero if $\left(X_{\tilde{n}}^{(k)}, d_{i}\right) \neq\left(A_{2 n}^{(2)}, 1\right)$ or $2 \mid s+r_{1}+r_{2}$, and depends only on $s+r_{1}+r_{2}$ otherwise; 
(v) $f_{i, L_{+}}\left(\left[\left[x_{j, s}^{+}, x_{i, r_{1}}^{+}\right], x_{i, r_{2}}^{+}\right]\right)$

$$
=a_{i j}\left[x_{j, s+r_{1}}^{+}, x_{i, r_{2}}^{+}\right]+a_{i j}\left[x_{j, s+r_{2}}^{+}, x_{i, r_{1}}^{+}\right]+B_{i i r_{2}}\left[x_{j, s}^{+}, x_{i, r_{1}+r_{2}}^{+}\right]
$$

(we can and do suppose $\tilde{d}_{j} \mid s$ and $\tilde{d}_{i} \mid r_{1}, r_{2}$ ).

Let us distinguish three cases:

If $k=2$ and $X_{\tilde{n}}^{(k)} \neq A_{2 n}^{(2)}$, then $x_{j, s+1}^{+}=0$, so that

$$
f_{i, L_{+}}\left(\left[\left[x_{j, s}^{+}, x_{i, 1}^{+}\right], x_{i, 0}^{+}\right]\right)=-2\left[x_{j, s}^{+}, x_{i, 1}^{+}\right]+2\left[x_{j, s}^{+}, x_{i, 1}^{+}\right]=0
$$

and

$$
\begin{aligned}
& f_{i, L_{+}}\left(\left[\left[x_{j, s}^{+}, x_{i, 1}^{+}\right], x_{i, 1}^{+}\right]+\left[\left[x_{j, s+2}^{+}, x_{i, 0}^{+}\right], x_{i, 0}^{+}\right]\right) \\
& \quad=2\left[x_{j, s}^{+}, x_{i, 2}^{+}\right]-2\left[x_{j, s+2}^{+}, x_{i, 0}^{+}\right]-2\left[x_{j, s+2}^{+}, x_{i, 0}^{+}\right]+2\left[x_{j, s+2}^{+}, x_{i, 0}^{+}\right]=0 ;
\end{aligned}
$$

together with (i), (ii) and (iv) this implies the stability of $\left(x_{d}, x_{1,2}, t_{2}^{\prime}, t_{2}^{\prime \prime}\right)$ by the action of the $f_{l}$ 's $\left(l \in I_{0}\right)$.

$$
\begin{aligned}
& \text { If } X_{\tilde{n}}^{(k)}=A_{2 n}^{(2)} \text {, then } \\
& \begin{aligned}
f_{i, L_{+}}\left(\left[\left[x_{j, s}^{+}, x_{i, 1}^{+}\right], x_{i, 0}^{+}\right]+\right. & {\left.\left.\left[x_{j, s}^{+}, x_{i, 0}^{+}\right], x_{i, 1}^{+}\right]\right) } \\
= & -2\left[x_{j, s+1}^{+}, x_{i, 0}^{+}\right]-2\left[x_{j, s}^{+}, x_{i, 1}^{+}\right]+2\left[x_{j, s}^{+}, x_{i, 1}^{+}\right] \\
& -2\left[x_{j, s}^{+}, x_{i, 1}^{+}\right]-2\left[x_{j, s+1}^{+}, x_{i, 0}^{+}\right]+6\left[x_{j, s}^{+}, x_{i, 1}^{+}\right]=0 ;
\end{aligned}
\end{aligned}
$$

together with (i), (ii) and (iv) this implies the stability of $\left(x_{d}, x_{1,2}, s_{2}\right)$ by the action of the $f_{l}$ 's.

If $k=3$, then

$$
\begin{aligned}
f_{i, L_{+}}\left(\left[\left[x_{j, s}^{+}, x_{i, r_{1}}^{+}\right], x_{i, r_{2}}^{+}\right]\right) & \begin{cases}(-6+2)\left[x_{j, s}^{+}, x_{i, r_{1}+r_{2}}^{+}\right] & \text {if } 3\left|r_{1}, 3\right| r_{2}, \\
(-3+2)\left[x_{j, s}^{+}, x_{i, r_{1}+r_{2}}^{+}\right] & \text {if } 3 \nmid r_{1}, 3 \mid r_{2} \text { or } 3 \mid r_{1}, 3 \nmid r_{2}, \\
2\left[x_{j, s}^{+}, x_{i, r_{1}+r_{2}}^{+}\right] & \text {if } 3 \nmid r_{1}, 3 \nmid r_{2} ;\end{cases}
\end{aligned}
$$

in particular

$$
\begin{array}{r}
f_{i, L_{+}}\left(\left[\left[x_{j, s}^{+}, x_{i, 1}^{+}\right], x_{i, 1}^{+}\right]+2\left[\left[x_{j, s}^{+}, x_{i, 2}^{+}\right], x_{i, 0}^{+}\right]\right)=(2-2)\left[x_{j, s}^{+}, x_{i, 2}^{+}\right]=0, \\
f_{i, L_{+}}\left(2\left[\left[x_{j, s}^{+}, x_{i, 2}^{+}\right], x_{i, 1}^{+}\right]+\left[\left[x_{j, s+3}^{+}, x_{i, 0}^{+}\right], x_{i, 0}^{+}\right]\right)=4\left[x_{j, s}^{+}, x_{i, 3}^{+}\right]-4\left[x_{j, s+3}^{+}, x_{i, 0}^{+}\right]=0, \\
f_{i, L_{+}}\left(\left[\left[x_{j, s}^{+}, x_{i, 2}^{+}\right], x_{i, 2}^{+}\right]+2\left[\left[x_{j, s+3}^{+}, x_{i, 1}^{+}\right], x_{i, 0}^{+}\right]\right) 2\left[x_{j, s}^{+}, x_{i, 4}^{+}\right]-2\left[x_{j, s+3}^{+}, x_{i, 1}^{+}\right]=0,
\end{array}
$$

which, together with (i), (ii) and (iv), implies the stability of $\left(x_{d}, x_{1,2}, t_{3}^{\prime}, t_{3}^{\prime \prime}, t_{3}^{\prime \prime \prime}\right)$ by the action of the $f_{l}$ 's.

If $k=3$ and $a_{i j}=-3$ then:

(vi) $f_{j, L_{+}}\left(\left[\left[\left[x_{j, s}^{+}, x_{i, r_{1}}^{+}\right], x_{i, r_{2}}^{+}\right], x_{i, r_{3}}^{+}\right]\right)=-a_{j i}\left[\left[x_{i, s+r_{1}}^{+}, x_{i, r_{2}}^{+}\right], x_{i, r_{3}}^{+}\right]=0$; 
if moreover $3 \nmid r$ then:

(vii) $f_{i, L_{+}}\left(\left[\left[\left[x_{j, s}^{+}, x_{i, r}^{+}\right], x_{i, 0}^{+}\right], x_{i, 0}^{+}\right]\right)=\left(2 a_{i j}+3 a_{i i}\right)\left[\left[x_{j, s}^{+}, x_{i, r}^{+}\right], x_{i, 0}^{+}\right]=0$.

Clauses (vi) and (vii), together with (i) and (ii), imply the stability of $\left(x_{d}, x_{1,2}, x_{3}, u_{3}^{\prime}, u_{3}^{\prime \prime}\right)$ by the action of the $f_{l}$ 's.

Finally if $i \neq j$ it is well known that

(viii) $f_{l, L_{+}}\left(\left(\operatorname{ad} x_{i, 0}^{+}\right)^{1-a_{i j}} x_{j, s}^{+}\right)=0$,

which implies the stability of (serre) by the action of the $f_{l}$ 's.

Definition 8.11. Let $e_{i, L}, f_{i, L}, h_{i, L}: L \rightarrow L$ be defined by:

$$
\begin{aligned}
\left.e_{i, L}\right|_{L_{0} \oplus L_{+}} & =\operatorname{ad}_{L_{0} \oplus L_{+}} x_{i, 0}^{+} \text {and }\left.f_{i, L}\right|_{L_{-} \oplus L_{0}}=\operatorname{ad}_{L_{-} \oplus L_{0}} x_{i, 0}^{-} \text {(see Remark 8.6(iii)); } \\
\left.f_{i, L}\right|_{L_{+}} & =f_{i, L_{+}} \quad \text { and }\left.\quad e_{i, L}\right|_{L_{-}}=e_{i, L_{-}} \quad(\text { see Definition 8.9); } \\
h_{i, L} & =\left(h_{i, 0}\right)_{L} \quad(\text { see Remark 8.6(v)). }
\end{aligned}
$$

Definition 8.12. Define $\tilde{\mathfrak{g}}_{0}$ to be the Lie algebra generated by $\left\{e_{i}, f_{i}, h_{i} \mid i \in I_{0}\right\}$ with relations

$$
\left[h_{i}, h_{j}\right]=0,\left[h_{i}, e_{j}\right]=a_{i j} e_{j},\left[h_{i}, f_{j}\right]=-a_{i j} f_{j},\left[e_{i}, f_{j}\right]=\delta_{i, j} h_{i} \quad \forall i, j \in I_{0} .
$$

Lemma 8.13. (i) $e_{i, L}, f_{i, L}: L \rightarrow L$ are homogeneous linear maps, hence locally nilpotent;

(ii) $\sigma \circ f_{i, L} \circ \sigma=e_{i, L}$;

(iii) $\left[e_{i, L}, f_{j, L}\right]=\delta_{i, j} h_{i, L}$;

(iv) $L$ is a $\tilde{\mathfrak{g}}_{0}$-module.

Proof. (i) follows from Remark 8.8(ii), and (ii) from Remark 8.6(ii) and from Definitions 8.9 and 8.11.

(iii) By (ii) and Remark 8.6(v) it is enough to prove the identity on $L_{0} \oplus L_{+}$. By homogeneity $e_{i, L} \circ f_{j, L}$ and $f_{j, L} \circ e_{i, L}$ map $L_{0}$ to $L_{\alpha_{i}-\alpha_{j}} \subseteq L_{0}$, and in particular to $\{0\}$ if $i \neq j$ while $\left.e_{i, L} \circ f_{i, L}\right|_{L_{0}}=\left.\sigma \circ e_{i, L} \circ f_{i, L} \circ \sigma\right|_{L_{0}}=\left.f_{i, L} \circ e_{i, L}\right|_{L_{0}}$ because $\left.\sigma\right|_{L_{0}}=\operatorname{id}_{L_{0}}$; hence $\left.\left[e_{i, L}, f_{j, L}\right]\right|_{L_{0}}=0=\left.\delta_{i, j}\left(h_{i}\right)_{L}\right|_{L_{0}}$; on the other hand, since $\left.f_{j, L}\right|_{L_{+}}=f_{j, L_{+}}: L_{+} \rightarrow L_{0} \oplus L_{+}$is a derivation,

$$
\begin{aligned}
\left.f_{j, L} \circ e_{i, L}\right|_{L_{+}} & =f_{j, L} \circ \operatorname{ad}_{L_{+}} x_{i, 0}^{+} \\
& =\left.\left(\operatorname{ad}_{L_{0} \oplus L_{+}}\left(f_{j, L}\left(x_{i, 0}^{+}\right)\right)+\left(\operatorname{ad}_{L_{0} \oplus L_{+}} x_{i, 0}^{+}\right) \circ f_{j, L}\right)\right|_{L_{+}} \\
& =\left.\left(-\delta_{i j} \operatorname{ad}_{L_{0} \oplus L_{+}} h_{i, 0}+e_{i, L} \circ f_{j, L}\right)\right|_{L_{+}}=\left.\left(-\delta_{i j}\left(h_{i, 0}\right)_{L}+e_{i, L} \circ f_{j, L}\right)\right|_{L_{+}} \\
& =\left.\left(-\delta_{i j} h_{i, L}+e_{i, L} \circ f_{j, L}\right)\right|_{L_{+}},
\end{aligned}
$$

which is the claim. 
(iv) is a consequence of (iii) together with Proposition 8.6(v) and Remark $8.8(\mathrm{i})$.

Lemma 8.14. Let $\rho: \tilde{\mathfrak{g}}_{0} \rightarrow \mathfrak{g l}(M)$ be a $\tilde{\mathfrak{g}}_{0}$-module structure on $M$ with weight space decomposition $M=\bigoplus_{\alpha \in \mathfrak{h}_{0}^{*}} M_{\alpha}\left(\left.\rho(h)\right|_{M_{\alpha}}=\alpha(h) \operatorname{id}_{M_{\alpha}}\right.$ for all $h \in \mathfrak{h}_{0}$, remarking that $\left.\mathfrak{h}_{0} \hookrightarrow \tilde{\mathfrak{g}}_{0}\right)$ and suppose that $\rho\left(e_{i}\right)$ and $\rho\left(f_{i}\right)$ are locally nilpotent. Then $M$ is a $\mathfrak{g}_{0}$-module.

Proof. Let $i \neq j \in I_{0}$; we want to prove that $\rho\left(\operatorname{ad}\left(e_{i}\right)^{1-a_{i j}}\left(e_{j}\right)\right)=0$ and $\rho\left(\operatorname{ad}\left(f_{i}\right)^{1-a_{i j}}\left(f_{j}\right)\right)=0$.

(a) Given $x \in M$ homogeneous, the subspace $M_{x}=\left\langle\rho\left(e_{i}\right)^{r}(x), \rho\left(f_{i}\right)^{r}(x) \mid r \in \mathbb{N}\right\rangle$ is finite-dimensional and $e_{i}, f_{i}, h_{i}$-stable;

(b) for $\tilde{M} \subseteq M$ finite-dimensional there exists $r \in \mathbb{N}$ such that $\left.\rho\left(e_{i}\right)^{r}\right|_{\tilde{M}}=0$; in particular there is $r_{x} \in \mathbb{N}$ such that $\left.\rho\left(e_{i}\right)^{r_{x}}\right|_{M_{x}}=0,\left.\rho\left(e_{i}\right)^{r_{x}}\right|_{\rho\left(e_{j}\right)\left(M_{x}\right)}=0$;

(c) for $r \in \mathbb{N}$ and $\rho\left(\operatorname{ad}\left(e_{i}\right)^{r}\left(e_{j}\right)\right)=\sum_{u=0}^{r}\left(\begin{array}{l}r \\ u\end{array}\right) \rho\left(e_{i}\right)^{r-u} \rho\left(e_{j}\right) \rho\left(e_{i}\right)^{u}$; in particular if $r \geq 2 r_{x}-1$ then $\left.\rho\left(\operatorname{ad}\left(e_{i}\right)^{r}\left(e_{j}\right)\right)\right|_{M_{x}}=0 ;$

(d) for $r \in \mathbb{N},\left[e_{i}, \operatorname{ad}\left(e_{i}\right)^{r}\left(e_{j}\right)\right]=\operatorname{ad}\left(e_{i}\right)^{r+1}\left(e_{j}\right)$ and $\left[f_{i}, \operatorname{ad}\left(e_{i}\right)^{r}\left(e_{j}\right)\right]=-r\left(a_{i j}+\right.$ $r-1) \operatorname{ad}\left(e_{i}\right)^{r-1}\left(e_{j}\right)$

(e) let $Y=\left\{r \in \mathbb{N}\left|\rho\left(\operatorname{ad}\left(e_{i}\right)^{r}\left(e_{j}\right)\right)\right|_{M_{x}}=0\right\}$; then $2 r_{x}-1 \in Y \neq \emptyset, r \in Y \Rightarrow$ $r+1 \in Y$ and $r \in Y \backslash\left\{0,1-a_{i j}\right\} \Rightarrow r-1 \in Y$; in particular $1-a_{i j} \in Y$ and $\rho\left(\operatorname{ad}\left(e_{i}\right)^{1-a_{i j}}\left(e_{j}\right)\right)(x)=0$.

Thus $\rho\left(\operatorname{ad}\left(e_{i}\right)^{1-a_{i j}}\left(e_{j}\right)\right)=0$. Composing $\rho$ with the Lie automorphism of $\mathfrak{g}_{0}$ defined by $e_{i} \mapsto-f_{i}, f_{i} \mapsto-e_{i}, h_{i} \mapsto-h_{i}$, we conclude that also $\rho\left(\operatorname{ad}\left(f_{i}\right)^{1-a_{i j}}\left(f_{j}\right)\right)=0$.

Corollary 8.15. $L$ is a $\mathfrak{g}_{0}$-module; $L^{(r)}$ is a $\mathfrak{g}_{0}$-module for all $r \in \mathbb{N}$.

Proof. The claim is a straightforward consequence of Remark 8.6(vi), of Lemma 8.13(i)\&(iv) and of Lemma 8.14.

Lemma 8.16. Let $\mathfrak{g}$ be a Lie algebra, $\mathfrak{h} \subseteq \mathfrak{g}$ a subalgebra, $M$ a $\mathfrak{g}$-module with $M=\bigoplus_{\alpha \in \mathfrak{h}^{*}} M_{\alpha}, M_{\alpha}=\{m \in M \mid h . m=\alpha(h) m$ for all $h \in \mathfrak{h}\}$. Let $\tau \in \operatorname{Aut}_{\text {Lie }}(\mathfrak{g})$ and $\varphi \in \mathrm{GL}(M)$ be such that:

(i) $\tau(\mathfrak{h})=\mathfrak{h}$;

(ii) $\varphi(y . m)=\tau(y) \cdot \varphi(m)$ for all $y \in \mathfrak{g}$ and $m \in M$.

Then $\tau$. $=\left(\left.\tau\right|_{\mathfrak{h}} ^{-1}\right)^{*} \in \mathrm{GL}\left(\mathfrak{h}^{*}\right)$ and $\varphi\left(M_{\alpha}\right)=M_{\tau . \alpha}$ for all $\alpha \in \mathfrak{h}^{*}$. In particular $P_{M}=\left\{\alpha \in \mathfrak{h}^{*} \mid M_{\alpha} \neq\{0\}\right\}$ is $\tau$.-stable and $\operatorname{dim} M_{\alpha}=\operatorname{dim} M_{\tau . \alpha}$ for all $\alpha \in P_{M}$.

Lemma 8.17. Let $\mathfrak{g}$ be a Lie algebra, $M$ be a $\mathfrak{g}$-module and $x \in \mathfrak{g}$ be such that $\operatorname{ad} x$ and $x_{M}$ are nilpotent $\left(x_{M}\right.$ denotes the map $\left.m \mapsto x \cdot m\right), \tau^{(x)}=\exp (\operatorname{ad} x)$ 
and $\varphi^{(x)}=\exp \left(x_{M}\right)$. Then $\tau^{(x)} \in \operatorname{Aut}_{\text {Lie }}(\mathfrak{g}), \varphi^{(x)} \in \operatorname{GL}(M)$ and $\varphi^{(x)}(y \cdot m)=$ $\tau^{(x)}(y) \cdot \varphi^{(x)}(m)$ for all $y \in \mathfrak{g}$ and $m \in M$. Moreover if $x_{1}, \ldots, x_{r} \in \mathfrak{g}$ are such that ad $x_{i}$ and $\left(x_{i}\right)_{M}$ are nilpotent and we set $\tau=\tau^{\left(x_{1}\right)} \circ \cdots \circ \tau^{\left(x_{r}\right)}$ and $\varphi=\varphi^{\left(x_{1}\right)} \circ \cdots \circ \varphi^{\left(x_{r}\right)}$, we still have $\tau \in \operatorname{Aut}_{\text {Lie }}(\mathfrak{g}), \varphi \in \operatorname{GL}(M)$ and $\varphi(y . m)=$ $\tau(y) . \varphi(m)$ for all $y \in \mathfrak{g}$ and $m \in M$.

Proof. This is a straightforward consequence of the well known identity

$$
x_{M}^{n}(y . m)=\sum_{r=0}^{n}\left(\begin{array}{l}
n \\
r
\end{array}\right)(\operatorname{ad} x)^{r}(y) \cdot x_{M}^{n-r}(m) .
$$

Remark 8.18. For all $r \in \mathbb{N}$ let us consider the $\mathfrak{g}_{0}$-module $L^{(r)}$ and the elements $e_{i}, f_{i} \in \mathfrak{g}_{0}$. Let

$$
\begin{aligned}
\tau_{i} & =\exp \left(\operatorname{ad} e_{i}\right) \exp \left(-\operatorname{ad} f_{i}\right) \exp \left(\operatorname{ad} e_{i}\right), \\
\varphi_{i} & =\exp \left(\left.e_{i, L}\right|_{L_{r}}\right) \exp \left(-\left.f_{i, L}\right|_{L_{r}}\right) \exp \left(\left.e_{i, L}\right|_{L_{r}}\right) .
\end{aligned}
$$

Then it is well known and obvious (from Lemmas 8.13(i), 8.15 and 8.17) that $\tau_{i} \in \operatorname{Aut}_{\text {Lie }}(\mathfrak{g})$ and $\varphi_{i} \in \mathrm{GL}(M)$ are well defined and $\varphi_{i}(y \cdot m)=\tau_{i}(y) \cdot \varphi_{i}(m)$ for all $y \in \mathfrak{g}_{0}$ and $m \in M$.

It is also well known (see $[\mathrm{K}])$ that $\tau_{i}\left(\mathfrak{h}_{0}\right)=\mathfrak{h}_{0}$ and in fact $\left.\tau_{i}\right|_{\mathfrak{h}_{0}}=s_{i} \in W_{0}$, hence by Lemma 8.16, $\left\{\alpha \in \mathfrak{h}_{0}^{*} \mid\left(L^{(r)}\right)_{\alpha}\left(=L_{\alpha+r \delta}\right) \neq\{0\}\right\}$ is $W_{0}$-stable and $\operatorname{dim} L_{w(\alpha)+r \delta}=\operatorname{dim} L_{\alpha+r \delta}$ for all $\alpha \in \mathfrak{h}_{0}^{*}, r \in \mathbb{N}$ and $w \in W_{0}$.

Recall that $\left\{\alpha \in \mathfrak{h}_{0}^{*} \mid L_{\alpha+r \delta} \neq\{0\}\right\} \subseteq Q_{0,+} \cup\left(-Q_{0,+}\right)$.

Lemma 8.19. Let $P \subseteq Q_{0,+} \cup\left(-Q_{0,+}\right)$ be $W_{0}$-stable. Then each $\alpha \in P$ is $W_{0}$-conjugate to an integer multiple of a simple root.

Proof. Let $\alpha \in P \backslash\{0\}$ and take $\beta \in W_{0} . \alpha \cap Q_{0,+}\left(\neq \emptyset\right.$ because there exists $\tilde{w} \in W_{0}$ such that $\left.\tilde{w}\left(Q_{0,+}\right)=-Q_{0,+}\right)$ of minimal height. Since $(\beta \mid \beta)>0$ there exists $i \in I_{0}$ such that $\left(\beta \mid \alpha_{i}\right)>0$, so that, by the choice of $\beta, s_{i}(\beta) \in-Q_{0,+}$. This implies $\beta$ is a multiple of $\alpha_{i}$.

Let us now come to our point.

Proposition 8.20. Given $\alpha \in Q_{0}$

$$
\operatorname{dim} L_{\alpha+r \delta} \leq \begin{cases}1 & \text { if } \alpha+r \delta \in \Phi \\ 0 & \text { otherwise }\end{cases}
$$

Proof. We have already proved (see Remark 8.18 and Lemma 8.19) that $\operatorname{dim} L_{\alpha+r \delta}=0$ if $\alpha \notin \bigcup_{h>0} h \Phi_{0}$. By Remark 8.18 it is then enough to prove the claim when $\alpha$ is an integer multiple of a simple root; but this is nothing but (D1)-(D3) (see Proposition 8.1). 
Corollary 8.21. $\left.\tilde{\psi}_{1}\right|_{L_{+}}: L_{+} \rightarrow\left(\mathfrak{g}_{+} \otimes \mathbb{C}[t]\right)^{\chi}$ is an isomorphism of Lie algebras.

Proof. The claim is a consequence of Propositions 7.7 and 8.20.

\section{$\S 9$. Conclusions}

In this section we point out and underline several consequences of Corollary 8.21. They include the main result ( $\psi$ is an isomorphism) together with other results which are worth presenting, both about the Drinfeld realization of affine quantum algebras and affine Kac-Moody algebras.

Theorem 9.1. $\psi: \mathcal{U}_{q}^{\mathrm{Dr}} \rightarrow \mathcal{U}_{q}^{\mathrm{DJ}}$ is an isomorphism. This means that the affine quantum algebras $\mathcal{U}_{q}^{\text {DJ }}$ (Drinfeld and Jimbo presentation) and $\mathcal{U}_{q}^{\text {Dr }}$ (Drinfeld realization) are different presentations of the same algebra $\mathcal{U}_{q}=\mathcal{U}_{q}\left(X_{\tilde{n}}^{(k)}\right)$, the affine quantum algebra of type $X_{\tilde{n}}^{(k)}$.

Theorem 9.2. The product induces an isomorphism

$$
\mathcal{U}_{q}^{\mathrm{Dr},-} \otimes \mathcal{U}_{q}^{\mathrm{Dr}, 0} \otimes \mathcal{U}_{q}^{\mathrm{Dr},+} \cong \mathcal{U}_{q}^{\mathrm{Dr}}=\mathcal{U}_{q}
$$

(triangular decomposition of the Drinfeld realization, or Drinfeld triangular decomposition of the affine quantum algebra).

As remarked above (see Remark 5.3), the Drinfeld triangular decomposition is essentially different from the Drinfeld and Jimbo triangular decomposition (Remark 2.2(ii)). Their precise connection is described below.

Proposition 9.3. (i) $\mathcal{U}_{q}^{\mathrm{Dr},+} \cap \mathcal{U}_{q}^{\mathrm{DJ},+}=\mathcal{U}_{q}^{\mathrm{Dr},+,+}$; it is the $\mathbb{C}(q)$-linear span of the ordered monomials in the $E_{r \delta+\alpha}$ 's with $\alpha \in Q_{0,+}$ and $r \geq 0$;

(ii) $\mathcal{U}_{q}^{\mathrm{Dr}, 0} \cap \mathcal{U}_{q}^{\mathrm{DJ},+}=\mathcal{U}_{q}^{\mathrm{Dr}, 0,+}$;

(iii) $\tilde{\mathcal{U}}_{q}^{\mathrm{Dr},-} \cap \mathcal{U}_{q}^{\mathrm{DJ},+}$ is the $\mathbb{C}(q)$-linear span of the ordered monomials in the $E_{r \delta-\alpha}$ 's with $\alpha \in Q_{0,+}$ and $r>0$;

(iv) $\mathcal{U}_{q}^{\mathrm{DJ},+} \cong\left(\tilde{\mathcal{U}}_{q}^{\mathrm{Dr},-} \cap \mathcal{U}_{q}^{\mathrm{DJ},+}\right) \otimes_{\mathbb{C}(q)} \mathcal{U}_{q}^{\mathrm{Dr}, 0,+} \otimes_{\mathbb{C}(q)} \mathcal{U}_{q}^{\mathrm{Dr},+,+}$;

(v) $\mathcal{U}_{q}^{\mathrm{Dr},+} \cap \tilde{\mathcal{U}}_{q}^{\mathrm{DJ},-}$ is the $\mathbb{C}(q)$-linear span of the ordered monomials in the $F_{r \delta-\alpha} K_{r \delta-\alpha}$ 's with $\alpha \in Q_{0,+}$ and $r>0$;

(vi) $\left(X_{i, r}^{+} \mid i \in I_{0}, r<0\right)=\mathcal{U}_{q}^{\mathrm{Dr},+} \cap \tilde{\mathcal{U}}_{q}^{\mathrm{DJ},-}$ in case $A_{1}^{(1)}$ and $\left(X_{i, r}^{+} \mid i \in I_{0}, r<0\right)$ $\subsetneq \mathcal{U}_{q}^{\mathrm{Dr},+} \cap \tilde{\mathcal{U}}_{q}^{\mathrm{DJ},-}$ otherwise

(vii) $\mathcal{U}_{q}^{\mathrm{Dr},+} \cap \tilde{\mathcal{U}}_{q}^{\mathrm{DJ},-} \subsetneq\left(X_{i, r}^{+} \mid i \in I_{0}, r \leq 0\right)$;

(viii) $\mathcal{U}_{q}^{\mathrm{Dr},+}=\mathcal{U}_{q}^{\mathrm{Dr},+,+} \otimes\left(\mathcal{U}_{q}^{\mathrm{Dr},+} \cap \tilde{\mathcal{U}}_{q}^{\mathrm{DJ},-}\right)$.

Proof. With the notation of Corollary 5.5 we have $\mathcal{U}_{q}^{\mathrm{DJ},+}=U_{-} \otimes U_{0} \otimes U_{+}$with $U_{-} \subseteq \tilde{\mathcal{U}}_{q}^{\mathrm{Dr},-}, U_{0} \subseteq \mathcal{U}_{q}^{\mathrm{Dr}, 0}, U_{+} \subseteq \mathcal{U}_{q}^{\mathrm{Dr},+}$, which implies (i)-(iv); (v) is equivalent to (iii). 
In (vi) the inclusions are obvious, as is the claim in case $A_{1}^{(1)}$; in the other cases there exist $i, j \in I_{0}$ such that $\delta-\left(\alpha_{i}+\alpha_{j}\right)$ is a root, so $F_{\delta-\left(\alpha_{i}+\alpha_{j}\right)} \in \mathcal{U}_{q}^{\mathrm{Dr},+} \cap \tilde{\mathcal{U}}_{q}^{\mathrm{DJ},-}$ while $\left(X_{i, r}^{+} \mid i \in I_{0}, r<0\right)_{-\delta+\left(\alpha_{i}+\alpha_{j}\right)}=(0)$.

In (vii) the inequality is obvious $\left(X_{i, 0}^{+} \notin \mathcal{U}_{q}^{\mathrm{Dr},+} \cap \tilde{\mathcal{U}}_{q}^{\mathrm{DJ},-}\right)$; for the inclusion, consider the subalgebra of $\mathcal{U}_{q}$ generated by $\left(\mathcal{U}_{q}^{\mathrm{Dr},+} \cap \tilde{\mathcal{U}}_{q}^{\mathrm{DJ},-}\right)$ and by the $X_{i, 0}^{+}$'s; it is isomorphic to $\left(\mathcal{U}_{q}^{\mathrm{Dr},+} \cap \tilde{\mathcal{U}}_{q}^{\mathrm{DJ},-}\right) \otimes\left(X_{i, 0}^{+} \mid i \in I_{0}\right)$, thanks to the triangular decomposition of $\mathcal{U}_{q}^{\mathrm{DJ}}$ and to the fact that for all $x \in\left(\mathcal{U}_{q}^{\mathrm{Dr},+} \cap \tilde{\mathcal{U}}_{q}^{\mathrm{DJ},-}\right)_{\alpha}, i \in I_{0}$ we have $\left[X_{i, 0}^{+}, x\right]_{q^{\left(\alpha \mid \alpha_{i}\right)}} \in \mathcal{U}_{q}^{\mathrm{Dr},+} \cap \tilde{\mathcal{U}}_{q}^{\mathrm{DJ},-}$, which implies $\left(\mathcal{U}_{q}^{\mathrm{Dr},+} \cap \tilde{\mathcal{U}}_{q}^{\mathrm{DJ},-}\right) \otimes\left(X_{i, 0}^{+} \mid i \in I_{0}\right)$ is not only a $\left(\mathcal{U}_{q}^{\mathrm{Dr},+} \cap \tilde{\mathcal{U}}_{q}^{\mathrm{DJ},-}\right)$-module, but also stable by left multiplication by the $X_{i, 0}^{+}$'s, hence a subalgebra of $\mathcal{U}_{q}$; but of course it contains $\left(X_{i, r}^{+} \mid i \in I_{0}, r \leq 0\right)$, so in order to prove that $\left(\mathcal{U}_{q}^{\mathrm{Dr},+} \cap \tilde{\mathcal{U}}_{q}^{\mathrm{DJ},-}\right) \otimes\left(X_{i, 0}^{+} \mid i \in I_{0}\right)$ and $\left(X_{i, r}^{+} \mid i \in I_{0}, r \leq 0\right)$ are equal (which is the claim), it is enough to compare the dimensions of their homogeneous components: for all $m \geq 0$ and $\alpha \in Q_{0,+}$.

$$
\begin{aligned}
\operatorname{dim}\left(\left(\mathcal{U}_{q}^{\mathrm{Dr},+} \cap \tilde{\mathcal{U}}_{q}^{\mathrm{DJ},-}\right) \otimes\left(X_{i, 0}^{+} \mid i \in I_{0}\right)\right)_{\alpha-m \delta} \\
\left.\left.=\sum_{\beta \in Q_{0,+}} \mathcal{U}_{q}^{\mathrm{Dr},+} \cap \tilde{\mathcal{U}}_{q}^{\mathrm{DJ},-}\right)_{\alpha-m \delta-\beta} X_{i, 0}^{+} \mid i \in I_{0}\right)_{\beta} \\
=\#\left\{\left(m_{1} \delta-\gamma_{1} \preceq \cdots \preceq m_{s} \delta-\gamma_{s}\right) \mid m_{u}>0, \gamma_{u} \in Q_{0,+}, \sum_{u} m_{u}=m, \sum_{u} \gamma_{u}=\alpha-\beta\right\} \\
\cdot \#\left\{\left(\gamma_{1}^{0} \preceq \cdots \preceq \gamma_{\tilde{s}}^{0}\right) \mid \gamma_{u}^{0} \in Q_{0,+}, \sum_{u} \gamma_{u}^{0}=\beta\right\} \\
=\#\left\{\left(m_{1} \delta+\gamma_{1} \preceq \cdots \preceq m_{s} \delta+\gamma_{s}\right) \mid m_{u}>0, \gamma_{u} \in Q_{0,+}, \sum_{u} m_{u}=m, \sum_{u} \gamma_{u}=\alpha-\beta\right\} \\
\cdot \#\left\{\left(\gamma_{1}^{0} \preceq \cdots \preceq \gamma_{\tilde{s}}^{0}\right) \mid \gamma_{u}^{0} \in Q_{0,+}, \sum_{u} \gamma_{u}^{0}=\beta\right\} \\
=\#\left\{\left(m_{1} \delta+\gamma_{1} \preceq \cdots \preceq m_{s} \delta+\gamma_{s}\right) \mid m_{u} \geq 0, \gamma_{u} \in Q_{0,+}, \sum_{u} m_{u}=m, \sum_{u} \gamma_{u}=\alpha\right\} \\
=\operatorname{dim} \mathcal{U}_{q, m \delta+\alpha}^{\mathrm{Dr},+,+}=\operatorname{dim}\left(X_{i, r}^{+} \mid i \in I_{0}, r \geq 0\right)_{m \delta+\alpha}=\operatorname{dim}\left(X_{i, r}^{+} \mid i \in I_{0}, r \leq 0\right)_{-m \delta+\alpha} ;
\end{aligned}
$$

this chain of equalities follows from the PBW-bases of $\mathcal{U}_{q}^{\mathrm{Dr},+} \cap \tilde{\mathcal{U}}_{q}^{\mathrm{DJ},-}($ see $(\mathrm{v}))$, of $\left(X_{i, 0}^{+} \mid i \in I_{0}\right)$ and of $\mathcal{U}_{q}^{\mathrm{Dr},+,+}$ (see (i)) and from the isomorphism between $\left(X_{i, r}^{+} \mid i \in I_{0}, r \leq 0\right)$ and $\left(X_{i, r}^{+} \mid i \in I_{0}, r \geq 0\right)$ (see [Da1]).

(viii) Thanks to (i) and to Remarks $2.3(\mathrm{x})$ and 3.5 (vii) the claim follows by remarking that for $r \leq s \leq 0, \lambda^{N}\left(\beta_{r}\right) \in-Q_{+}$implies $\lambda^{N}\left(\beta_{s}\right) \in-Q_{+}$(hence $T_{\lambda}^{N}\left(E_{\beta_{r}}\right) \in \tilde{\mathcal{U}}_{q}^{\mathrm{DJ},-} \Rightarrow T_{\lambda}^{N}\left(E_{\beta_{s}}\right) \in \tilde{\mathcal{U}}_{q}^{\mathrm{DJ},-}$, by Remark $\left.2.3(\mathrm{vi})\right)$.

Theorem 9.4. (i) $\mathcal{U}_{q}^{\mathrm{Dr},+}$ is the $\mathbb{C}(q)$-algebra generated by $\left\{X_{i, r}^{+} \mid i \in I_{0}, r \in \mathbb{Z}\right\}$ with relations $\left(Z X^{+}\right)$and $(D R)$.

(ii) $\mathcal{U}_{q}^{\mathrm{Dr},+,+}$ is the $\mathbb{C}(q)$-algebra generated by $\left\{X_{i, r}^{+} \mid i \in I_{0}, r \in \mathbb{N}\right\}$ with relations $\left(Z X_{+}^{+}\right),\left(D R_{+}\right),\left(S_{+}\right),\left(U 3_{+}\right)$. 
(iii) The $\mathcal{A}$-subalgebra $\mathcal{U}_{\mathcal{A}}^{\mathrm{Dr},+}$ of $\mathcal{U}_{q}^{\mathrm{Dr}}$ generated by $\left\{X_{i, r}^{+} \mid i \in I_{0}, r \in \mathbb{Z}\right\}$ is the $\mathcal{A}$-algebra generated by $\left\{X_{i, r}^{+} \mid i \in I_{0}, r \in \mathbb{Z}\right\}$ with relations $\left(Z X^{+}\right)$and $(D R)$ and is a free $\mathcal{A}$-module: it is an integer form of $\mathcal{U}_{q}^{\mathrm{Dr},+}$.

(iv) The $\mathcal{A}$-subalgebra of $\mathcal{U}_{q}^{\mathrm{Dr}}$ generated by $\left\{X_{i, r}^{+} \mid i \in I_{0}, r \in \mathbb{N}\right\}$ is the $\mathcal{A}$-algebra generated by $\left\{X_{i, r}^{+} \mid i \in I_{0}, r \in \mathbb{N}\right\}$ with relations $\left(Z X_{+}^{+}\right),\left(D R_{+}\right),\left(S_{+}\right)$, $\left(U 3_{+}\right)$and it is a free $\mathcal{A}$-module: it is an integer form of $\mathcal{U}_{q}^{\text {Dr, },+,+}$.

Proof. (iv) is true by Corollary 6.4 and clearly implies (ii). Of course (iii) implies (i).

(iii) follows from (iv): Let $\mathcal{F}$ be the $\mathcal{A}$-algebra freely generated by $\left\{X_{i, r}^{+} \mid\right.$ $\left.i \in I_{0}, r \in \mathbb{Z}\right\}, \mathcal{I}$ the ideal of $\mathcal{F}$ defined by the relations $(D R), t: \mathcal{F} \rightarrow \mathcal{F}$ the $\mathcal{A}$-automorphism defined by $X_{i, r}^{+} \mapsto X_{i, r+\tilde{d}_{i}}^{+}, \bar{t}$ the $\mathcal{A}$-automorphism induced by $t$ on $\mathcal{F} / \mathcal{I}$, and $f: \mathcal{F} / \mathcal{I} \rightarrow \mathcal{U}_{q}^{\text {Dr }}$ the natural homomorphism. Consider also the natural homomorphism $j: \mathcal{F}_{+} / \mathcal{I}_{+} \rightarrow \mathcal{F} / \mathcal{I}$ (see Notation 6.1 and Remark 3.10(iii)).

Since of course $f \circ j=f_{+}$and $f \circ \bar{t}=\left(t_{1} \circ \cdots \circ t_{n}\right)^{-1} \circ f, f$ is injective thanks to Corollary 6.4(i) and to the fact that $\mathcal{F} / \mathcal{I}=\bigcup_{N \in \mathbb{N}} \bar{t}^{-N}\left(j\left(\mathcal{F}_{+} / \mathcal{I}_{+}\right)\right)$.

In order to prove that $\mathcal{F} / \mathcal{I}$ is free over $\mathcal{A}$ it is enough to remark that the image of the (injective) homomorphism $\psi \circ f$ is contained in $\mathcal{U}_{\mathcal{A}}^{\mathrm{DJ}}$ (see Remarks 2.4(iii) and 6.5), which is well known to be a free $\mathcal{A}$-module (see Remark 2.4(ii)).

Theorem 9.5. Let $\mathcal{U}_{\mathcal{A}}^{\mathrm{Dr},+}$ be as in Theorem $9.4(\mathrm{iii})$ and let $\mathcal{U}_{\mathcal{A}}^{\mathrm{Dr}}, \mathcal{U}_{\mathcal{A}}^{\mathrm{Dr},-}$ and $\mathcal{U}_{\mathcal{A}}^{\mathrm{Dr}, 0}$ be the $\mathcal{A}$-subalgebras of $\mathcal{U}_{q}^{\mathrm{Dr}}$ generated respectively by $\left\{X_{i, r}^{ \pm}, k_{i}^{ \pm 1}, C^{ \pm 1} \mid i \in I_{0}\right.$, $r \in \mathbb{Z}\},\left\{X_{i, r}^{-} \mid i \in I_{0}, r \in \mathbb{Z}\right\}$ and $\left\{H_{i, r}, k_{i}, C, \tilde{C} \mid i \in I_{0}, r \in \mathbb{Z}\right\}$, where

$$
H_{i, 0}=\frac{k_{i}-k_{i}^{-1}}{q_{i}-q_{i}^{-1}} \quad \text { and } \quad \tilde{C}=\frac{C-C^{-1}}{q-q^{-1}} .
$$

Then:

(i) $\mathcal{U}_{\mathcal{A}}^{\mathrm{Dr}}=\mathcal{U}_{\mathcal{A}}^{\mathrm{Dr},-} \otimes_{\mathcal{A}} \mathcal{U}_{\mathcal{A}}^{\mathrm{Dr}, 0} \otimes_{\mathcal{A}} \mathcal{U}_{\mathcal{A}}^{\mathrm{Dr},+}$;

(ii) $\mathcal{U}_{\mathcal{A}}^{\mathrm{Dr}, 0}=\mathcal{U}_{q}^{\mathrm{Dr}, 0} \cap \mathcal{U}_{\mathcal{A}}^{\mathrm{Dr}}$ and $\mathcal{U}_{\mathcal{A}}^{\mathrm{Dr}, \pm}=\mathcal{U}_{q}^{\mathrm{Dr}, \pm} \cap \mathcal{U}_{\mathcal{A}}^{\mathrm{Dr}}$;

(iii) $\mathcal{U}_{\mathcal{A}}^{\mathrm{Dr}, *}$ is an integer form of $\mathcal{U}_{q}^{\mathrm{Dr}, *}$; this means that it is a free $\mathcal{A}$-module and $\mathcal{U}_{q}^{\mathrm{Dr}, *}=\mathbb{C}(q) \otimes_{\mathcal{A}} \mathcal{U}_{\mathcal{A}}^{\mathrm{Dr}, *}$

(iv) $\mathcal{U}_{\mathcal{A}}^{\mathrm{Dr}, 0}$ is the $\mathcal{A}$-algebra generated by $\left\{H_{i, r}, k_{i}, C, \tilde{C} \mid i \in I_{0}, r \in \mathbb{Z}\right\}$ with relations $(Z H),(C U K)$,

$(C U H) \quad[\tilde{C}, x]=0, \quad\left[k_{i}, H_{j, 0}\right]=0, \quad\left[H_{i, 0}, H_{j, 0}\right]=0 \quad\left(i, j \in I_{0}\right)$,

$(I Q) \quad k_{i}\left(k_{i}-\left(q_{i}-q_{i}^{-1}\right) H_{i, 0}\right)=1, \quad C\left(C-\left(q-q^{-1}\right) \tilde{C}\right)=1$,

$(K Q H) \quad\left[H_{i, 0}, H_{j, r}\right]=0 \quad\left(i, j \in I_{0}, r \in \mathbb{Z}\right)$,

$(K H)$ and $(H H)$; 
(v) $\mathcal{U}_{\mathcal{A}}^{\mathrm{Dr}}$ is the $\mathcal{A}$-algebra generated by $\left\{X_{i, r}^{ \pm}, H_{i, r}, k_{i}, C, \tilde{C} \mid i \in I_{0}, r \in \mathbb{Z}\right\}$ with relations $\left(Z X^{ \pm}\right),(C U K),(C U H),(I Q),(K Q H),\left(K X^{ \pm}\right)$,

$\left(H Q X^{ \pm}\right) \quad H_{i, 0} X_{j, r}^{ \pm}=X_{j, r}^{ \pm}\left( \pm\left[a_{i j}\right]_{q_{i}} k_{i}+q_{i}^{\mp a_{i j}} H_{i, 0}\right)$,

$(H H),\left(H X^{ \pm}\right),(X X),\left(X 1_{\text {const }}^{ \pm}\right),\left(X 3_{\text {const }}^{ \pm}\right)$and $\left(S_{\text {const }}^{ \pm}\right)$;

(vi) $\mathcal{U}_{\mathcal{A}}^{\mathrm{Dr}}=\mathcal{U}_{\mathcal{A}}^{\mathrm{DJ}}$.

Proof. First of all note that $\mathcal{U}_{\mathcal{A}}^{\mathrm{Dr}, 0} \subseteq \mathcal{U}_{\mathcal{A}}^{\mathrm{Dr}}$, since $H_{i, 0}=\left[X_{i, 0}^{+}, X_{i, 0}^{-}\right]$for all $i \in I_{0}$ and $\tilde{C}=k_{i}\left(\left[X_{i, 1}^{+}, X_{i,-1}^{-}\right]-C H_{i, 0}\right)$ if $i \in I_{0}$ is such that $\tilde{d}_{i}=d_{i}=1$. Moreover $\mathcal{U}_{\mathcal{A}}^{\mathrm{Dr}, *} \subseteq \mathcal{U}_{q}^{\mathrm{Dr}, *}=\mathbb{C}(q) \otimes_{\mathcal{A}} \mathcal{U}_{\mathcal{A}}^{\mathrm{Dr}, *}$, so that $\mathcal{U}_{\mathcal{A}}^{\mathrm{Dr},-} \otimes_{\mathcal{A}} \mathcal{U}_{\mathcal{A}}^{\mathrm{Dr}, 0} \otimes_{\mathcal{A}} \mathcal{U}_{\mathcal{A}}^{\mathrm{Dr},+} \rightarrow \mathcal{U}_{\mathcal{A}}^{\mathrm{Dr}}$ is injective, thanks to Theorem 9.2.

Let $\mathcal{V}$ be the $\mathcal{A}$-algebra generated by $\left\{X_{i, r}^{ \pm}, H_{i, r}, k_{i}, C, \tilde{C} \mid i \in I_{0}, r \in \mathbb{Z}\right\}$ with relations $\left(Z X^{ \pm}\right),(C U K),(C U H),(I Q),(K Q H),\left(K X^{ \pm}\right),\left(H Q X^{ \pm}\right),(H H)$, $\left(H X^{ \pm}\right),(X X),\left(X 1_{\text {const }}^{ \pm}\right),\left(X 3_{\text {const }}^{ \pm}\right),\left(S_{\text {const }}^{ \pm}\right)$, and $\mathcal{V}^{+}, \mathcal{V}^{-}, \mathcal{V}^{0}$ be the $\mathcal{A}-$ subalgebras of $\mathcal{V}$ generated respectively by $\left\{X_{i, r}^{+} \mid i \in I_{0}, r \in \mathbb{Z}\right\},\left\{X_{i, r}^{-} \mid i \in I_{0}\right.$, $r \in \mathbb{Z}\}$ and $\left\{H_{i, r}, k_{i}, C, \tilde{C} \mid i \in I_{0}, r \in \mathbb{Z}\right\}$.

Let $\tilde{\mathcal{V}}^{0}$ be the $\mathcal{A}$-algebra generated by $\left\{H_{i, r}, k_{i}, C, \tilde{C} \mid i \in I_{0}, r \in \mathbb{Z}\right\}$ with relations $(Z H),(C U K),(C U H),(I Q),(K H),(K Q H),(H H)$, and $\tilde{\mathcal{V}}^{0,0}=$ $\mathcal{A}\left[k_{i}, C, H_{i, 0}, \tilde{C} \mid i \in I_{0}\right] / J$ where $J$ is the ideal generated by the relations $(I Q)$. Then:

(a) $\mathcal{V}^{*} \rightarrow \mathcal{U}_{\mathcal{A}}^{\mathrm{Dr}, *}$ is well defined and surjective $(* \in\{\emptyset, 0,+,-\})$.

(b) $\mathcal{V}^{-} \otimes \mathcal{V}^{0} \otimes \mathcal{V}^{+} \rightarrow \mathcal{V}$ is surjective, thanks to relations $(C U K),(C U H),\left(K X^{ \pm}\right)$, $\left(H Q X^{ \pm}\right),\left(H X^{ \pm}\right),(X X)$, so that the commutativity of the diagram

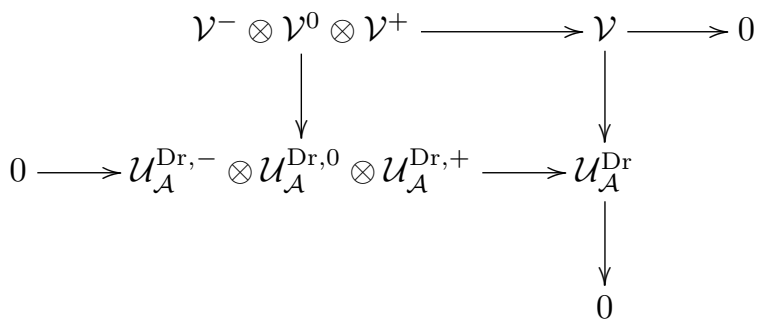

implies (i); (ii) follows from (i).

(c) $\tilde{\mathcal{V}}^{0,0} \cong \mathcal{U}_{\mathcal{A}}^{\mathrm{DJ}} \cap \mathcal{U}_{q}^{\mathrm{DJ}, 0}$ is a free $\mathcal{A}$-module (well known, see Remark 2.4(ii)\&(vi)), so that $\tilde{\mathcal{V}}^{0,0} \rightarrow \mathcal{U}_{\mathcal{A}}^{\mathrm{Dr}}$ is injective; moreover the (well defined) maps

$\mathcal{A}\left[H_{i, r} \mid i \in I_{0}, r<0\right] \otimes \tilde{\mathcal{V}}^{0,0} \otimes \mathcal{A}\left[H_{i, r} \mid i \in I_{0}, r>0\right] \rightarrow \tilde{\mathcal{V}}^{0} \quad$ and $\quad \tilde{\mathcal{V}}^{0} \rightarrow \mathcal{V}^{0}$ are surjective (thanks to relations $(C U K),(C U H),(K H),(K Q H)$ and $(H H))$, and the composition

$$
\mathcal{A}\left[H_{i, r} \mid i \in I_{0}, r<0\right] \otimes \tilde{\mathcal{V}}^{0,0} \otimes \mathcal{A}\left[H_{i, r} \mid i \in I_{0}, r>0\right] \rightarrow \tilde{\mathcal{V}}^{0} \rightarrow \mathcal{V}^{0} \rightarrow \mathcal{U}_{\mathcal{A}}^{\mathrm{Dr}, 0}
$$


is injective; in particular $\tilde{\mathcal{V}}^{0} \cong \mathcal{V}^{0} \cong \mathcal{U}_{\mathcal{A}}^{\mathrm{Dr}, 0}$ so that (iv) holds, and $\mathcal{U}_{\mathcal{A}}^{\mathrm{Dr}, 0}$ is a free $\mathcal{A}$-module; together with (i) and with Theorem 9.4(iii) this also implies (iii).

(d) $\mathcal{U}_{\mathcal{A}}^{\mathrm{Dr},+} \rightarrow \mathcal{V}$ is well defined (Remark 3.10 holds also on $\mathcal{A}$, see [Da1]) with image in $\mathcal{V}^{+}$, and $\mathcal{U}_{\mathcal{A}}^{\mathrm{Dr},+} \rightarrow \mathcal{V}^{+}$is obviously surjective: since

$$
\mathcal{U}_{\mathcal{A}}^{\mathrm{Dr},+} \rightarrow \mathcal{V}^{+} \rightarrow \mathcal{U}_{\mathcal{A}}^{\mathrm{Dr},+}
$$

is the identity we have $\mathcal{U}_{\mathcal{A}}^{\mathrm{Dr},+} \cong \mathcal{V}^{+}$; then the commutativity of the diagram

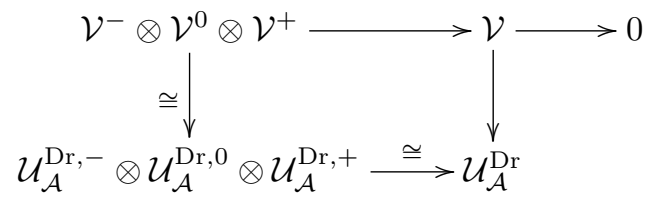

implies that $\mathcal{U}_{\mathcal{A}}^{\mathrm{Dr}} \cong \mathcal{V}$, that is, (v) holds.

(e) Since $\mathcal{U}_{\mathcal{A}}^{\mathrm{DJ}}$ is $t_{i}$-stable for all $i \in I_{0}$, we have $X_{i, r}^{ \pm} \in \mathcal{U}_{\mathcal{A}}^{\mathrm{DJ}}$ for all $(i, r) \in I_{\mathbb{Z}}$; it is also clear that $\mathcal{U}_{\mathcal{A}}^{\mathrm{Dr}, 0} \subseteq \mathcal{U}_{\mathcal{A}}^{\mathrm{DJ}}$, hence $\mathcal{U}_{\mathcal{A}}^{\mathrm{Dr}} \subseteq \mathcal{U}_{\mathcal{A}}^{\mathrm{DJ}}$; on the other hand clearly $C, K_{i}, E_{i} \in \mathcal{U}_{\mathcal{A}}^{\mathrm{Dr}}$ for all $i \in I_{0}, E_{0} \in \mathcal{U}_{\mathcal{A}}^{\mathrm{Dr}}$ (see [Da1]) and $F_{i} \in \mathcal{U}_{\mathcal{A}}^{\mathrm{Dr}}$ for all $i \in I$ since $\mathcal{U}_{\mathcal{A}}^{\mathrm{Dr}}$ is $\Omega$-stable; then $\mathcal{U}_{\mathcal{A}}^{\mathrm{DJ}} \subseteq \mathcal{U}_{\mathcal{A}}^{\mathrm{Dr}}$ and (vi) follows.

Theorem 9.6. Consider the affine Kac-Moody algebra

$$
\hat{\mathfrak{g}}=\hat{\mathfrak{g}}^{-} \oplus \hat{\mathfrak{g}}^{0} \oplus \hat{\mathfrak{g}}^{+}=\left(\mathfrak{g}_{-} \otimes \mathbb{C}\left[t^{ \pm 1}\right]\right)^{\chi} \oplus\left(\left(\mathfrak{g}_{0} \otimes \mathbb{C}\left[t^{ \pm 1}\right]\right)^{\chi} \oplus \mathbb{C} c\right) \oplus\left(\mathfrak{g}_{+} \otimes \mathbb{C}\left[t^{ \pm 1}\right]\right)^{\chi}
$$

\section{Then:}

(i) $\hat{\mathfrak{g}}^{+}$is the Lie algebra generated by $\left\{x_{i, r}^{+}\left|i \in I_{0}, \tilde{d}_{i}\right| r \in \mathbb{Z}\right\}$ with relations:

$$
\begin{gathered}
{\left[x_{i, r}^{+}, x_{j, s}^{+}\right] \text {depends only on } r+s \quad\left(i \neq j \in I_{0} \text { fixed }\right)} \\
{\left[x_{i, r}^{+}, x_{i, s}^{+}\right]=\left\{\begin{array}{r}
0 \\
\text { if }\left(X_{\tilde{n}}^{(k)}, d_{i}\right) \neq\left(A_{2 n}^{(2)}, 1\right) \text { or } 2 \mid r+s, \\
(-1)^{h}\left[x_{i, s+h+1}^{+}, x_{i, s+h}^{+}\right] \quad \text { if } r=s+2 h+1 ;
\end{array}\right.} \\
{\left[\left[x_{i, r_{1}}^{+}, x_{i, r_{2}}^{+}\right], x_{i, r_{3}}^{+}\right]=0 \quad\left(\left(r_{1}, r_{2}, r_{3}\right) \in \mathbb{N}^{3}\right) ;} \\
{\left[\left[x_{j, s}^{+}, x_{i, 1}^{+}\right], x_{i, 0}^{+}\right]=0,} \\
{\left[\left[x_{j, s}^{+}, x_{i, 1}^{+}\right], x_{i, 1}^{+}\right]=-\left[\left[x_{j, s+2}^{+}, x_{i, 0}^{+}\right], x_{i, 0}^{+}\right] \quad\left(k=2, a_{i j}=-2, X_{\tilde{n}}^{(k)} \neq A_{2 n}^{(2)}\right) ;} \\
{\left[\left[x_{j, s}^{+}, x_{i, 0}^{+}\right], x_{i, 1}^{+}\right]+\left[\left[x_{j, s+1}^{+}, x_{i, 0}^{+}\right], x_{i, 0}^{+}\right]=0 \quad\left(a_{i j}=-2, X_{\tilde{n}}^{(k)}=A_{2 n}^{(2)}\right) ;} \\
{\left[\left[x_{j, s}^{+}, x_{i, 1}^{+}\right], x_{i, 1}^{+}\right]=-2\left[\left[x_{j, s}^{+}, x_{i, 2}^{+}\right], x_{i, 0}^{+}\right] \quad\left(k=3, a_{i j}=-3\right) ;} \\
2\left[\left[x_{j, s}^{+}, x_{i, 2}^{+}\right], x_{i, 1}^{+}\right]=-\left[\left[x_{j, s+3}^{+}, x_{i, 0}^{+}\right], x_{i, 0}^{+}\right] \quad\left(k=3, a_{i j}=-3\right) ; \\
{\left[\left[x_{j, s}^{+}, x_{i, 2}^{+}\right], x_{i, 2}^{+}\right]=-2\left[\left[x_{j, s+3}^{+}, x_{i, 1}^{+}\right], x_{i, 0}^{+}\right] \quad\left(k=3, a_{i j}=-3\right) ;} \\
\left(\operatorname{ad} x_{i, 0}^{+}\right)^{1-a_{i j}}\left(x_{j, s}^{+}\right)=0 \quad\left(i \neq j \quad \text { with } k=1 \text { or } a_{i j} \geq-1\right) .
\end{gathered}
$$


(ii) $\hat{\mathfrak{g}}^{-}=\left(\hat{\mathfrak{g}}^{+}\right)^{\mathrm{op}}$.

(iii) $\hat{\mathfrak{g}}^{0}$ is the Lie algebra generated by $\left\{h_{i, r}, c\left|i \in I_{0}, \tilde{d}_{i}\right| r \in \mathbb{Z}\right\}$ with relations

$$
\left[c, h_{i, r}\right]=0, \quad\left[h_{i, r}, h_{j, s}\right]=\delta_{r+s, 0} \frac{r B_{i j r}}{d_{j}} c .
$$

(iv) $\hat{\mathfrak{g}}$ is the Lie algebra generated by $\left\{x_{i, r}^{+}, x_{i, r}^{-}, h_{i, r}, c\left|i \in I_{0}, \tilde{d}_{i}\right| r \in \mathbb{Z}\right\}$ with relations

$$
\begin{aligned}
{[c, a] } & =0 \quad \forall a, \\
{\left[h_{i, r}, h_{j, s}\right] } & =\delta_{r+s, 0} \frac{r B_{i j r}}{d_{j}} c, \\
{\left[h_{i, r}, x_{j, s}^{ \pm}\right] } & = \pm B_{i j r} x_{j, r+s}^{ \pm}, \\
{\left[x_{i, r}^{+}, x_{j, s}^{-}\right] } & =\delta_{i, j}\left(h_{i, r+s}+\delta_{r+s, 0} \frac{r-s}{2 d_{i}} c\right), \\
{\left[x_{i, r \pm 1}^{ \pm}, x_{i, r}^{ \pm}\right] } & =0 \quad\left(X_{\tilde{n}}^{(k)}=A_{1}^{(1)}\right), \\
{\left.\left[x_{i, r \pm 1}^{ \pm}, x_{i, r}^{ \pm}\right], x_{i, r}^{ \pm}\right] } & =0 \quad\left(X_{\tilde{n}}^{(k)}=A_{2}^{(2)}\right), \\
\left(\operatorname{ad} x_{i, r}^{ \pm}\right)^{1-a_{i j}}\left(x_{j, s}^{ \pm}\right) & =0 \quad\left(n>1, i \neq j \in I_{0}\right) .
\end{aligned}
$$

(Note that the relations $\left[h_{i, r}, h_{j, s}\right]=\delta_{r+s, 0} \frac{r B_{i j r}}{d_{j}} c$ depend on the others.)

Proof. Since

$$
\mathcal{U}(\hat{\mathfrak{g}})=\mathcal{U}_{\mathcal{A}}^{\mathrm{DJ}} /\left(q-1, K_{i}-1 \mid i \in I\right)=\mathcal{U}_{\mathcal{A}}^{\mathrm{Dr}} /\left(q-1, C-1, k_{i}-1 \mid i \in I_{0}\right)
$$

(see Remark 2.4(vii) and Theorem 9.5(vi)) the claims follow from Theorem 9.4(iii), Remark 3.5(ii) and Theorem 9.5(iv)\&(v).

\section{Acknowledgements}

I want to thank Velleda Baldoni for her support and advice and Hiraku Nakajima for his interest and attention.

\section{References}

[A] T. Akasaka, An integral PBW basis of the quantum affine algebra of type $A_{2}^{(2)}$, Publ. RIMS Kyoto Univ. 38 (2002), 803-894. Zbl 1038.17005 MR 1917164

[Be] J. Beck, Braid group action and quantum affine algebras, Comm. Math. Phys. 165 (1994), 555-568. Zbl 0807.17013 MR 1301623

[Bo] N. Bourbaki, Groupes et algèbres de Lie 4, 5, 6, Hermann, Paris, 1968. Zbl 0186.33001 MR 0240238

[Da1] I. Damiani, Drinfeld realization of affine quantum algebras: the relations, Publ. RIMS Kyoto Univ. 48 (2012), 661-733. Zbl 1297.17009 MR 2973398 
[Da2] The R-matrix for (twisted) affine quantum algebras, in Representations and quantizations (Shanghai, 1998), China High. Educ. Press, Beijing, 2000, 89-144. Zbl 1007.17012 MR 1802170

[Dr1] V. G. Drinfel'd, A new realization of Yangians and of quantum affine algebras, Dokl. Akad. Nauk SSSR 296 (1987), 13-17 (in Russian). Z Zbl 0667.16004 MR 0914215

[Dr2] Hopf algebras and the quantum Yang-Baxter equation, Dokl. Akad. Nauk SSSR 283 (1985), 1060-1064 (in Russian). Zbl 0588.17015 MR 0802128

[H] D. Hernandez, Representations of quantum affinizations and fusion product, Transform. Groups 10 (2005), 163-200. Zbl 1102.17009 MR 2195598

[Jm] M. Jimbo, A $q$-difference analogue of $U(\mathfrak{g})$ and the Yang-Baxter equation, Lett. Math. Phys. 10 (1985), 63-69. Zbl 0587.17004 MR 0797001

[K] V. G. Kac, Infinite dimensional Lie algebras, Birkhäuser, Boston, 1983. Z Zbl 0537.17001 MR 0739850

[LS] S. Z. Levendorskii and Ya. S. Soibel'man, Some applications of the quantum Weyl groups, J. Geom. Phys. 7 (1990), 241-254. MR 1120927

[L] G. Lusztig, Finite-dimensional Hopf algebras arising from quantized enveloping algebras, J. Amer. Math. Soc. 3 (1990), 257-296. Zbl 0695.16006 MR 1013053

[M] H. Matsumoto, Générateurs et relations des groupes de Weyl généralisés, C. R. Acad. Sci. Paris Sér. II 258 (1964), 3419-3422. Z Zbl 0128.25202 MR 0183818

[N] H. Nakajima, Quiver varieties and finite-dimensional representations of quantum affine algebras, J. Amer. Math. Soc. 14 (2001), 145-238. Zbl 0981.17016 MR 1808477 